Supporting Information

\title{
A General Copper-Catalyzed Vinylic Halogen Exchange Reaction
}

\author{
Antoine Nitelet and Gwilherm Evano* \\ Laboratoire de Chimie Organique, Service de Chimie et PhysicoChimie Organiques, Université \\ libre de Bruxelles (ULB), Avenue F. D. Roosevelt 50, CP160/06, 1050 Brussels, Belgium
}

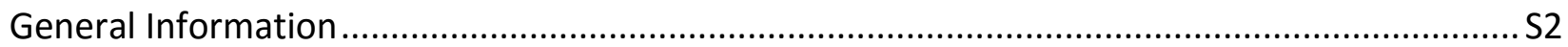

Experimental Procedures and Characterization Data: Unreported Starting Materials................S3

Experimental Procedures and Characterization Data:

Copper-Catalyzed I/Br $\rightarrow \mathrm{Cl}$ Exchange from Alkenyl lodides/Bromides ...................................S6

Experimental Procedures and Characterization Data:

Copper-Catalyzed I $\rightarrow$ Br Exchange from Alkenyl lodides ............................................................S18

Experimental Procedures and Characterization Data:

Copper-Catalyzed $\mathrm{Br} \rightarrow \mathrm{Cl}$ Exchange from gem-Dibromoalkenes .........................................S26

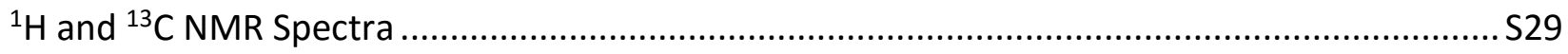




\section{General Information.}

All reactions were carried out in oven-dried glassware under an argon atmosphere employing standard techniques in handling air-sensitive materials.

All solvents were reagent grade. Tetrahydrofuran was freshly distilled from sodium/benzophenone under argon, dichloromethane was distilled from calcium hydride under argon. Dimethylsulfoxyde and dioxane were bought over molecular sieves in AcroSeal ${ }^{\circledR}$ bottles from Acros Organics and used as supplied. Copper(I) iodide (99,999\% purity) was purchased from Aldrich and used as supplied. All other reagents were used as supplied.

Reactions were magnetically stirred and monitored by thin layer chromatography using Merck-Kieselgel $60 \mathrm{~F}_{254}$ plates. Flash chromatography and filtrations were performed with silica gel 60 (particle size 40-63 $\mu \mathrm{m}$ ) supplied by Merck. Yields refer to chromatographically and spectroscopically pure compounds, unless otherwise stated.

Proton NMR spectra were recorded using an internal deuterium lock at ambient temperature on Brucker 300 and $400 \mathrm{MHz}$ spectrometers. Internal reference of $\delta_{\mathrm{H}} 7.26$ was used for $\mathrm{CDCl}_{3}$. Internal reference of $\delta_{\mathrm{H}} 2.50$ was used for $\left(\mathrm{CD}_{3}\right)_{2} \mathrm{SO}$. Data are presented as follows: chemical shift (in ppm on the $\delta$ scale relative to $\delta_{\mathrm{TMS}}=0$ ), multiplicity ( $\mathrm{s}=$ singlet, $\mathrm{d}=$ doublet, $\mathrm{t}=$ triplet, $\mathrm{q}=$ quartet, $\mathrm{m}=$ multiplet, $\mathrm{br} .=$ broad, app.= apparent), coupling constants $(\mathrm{J} / \mathrm{Hz})$ and integration. Resonances that are either partially or fully obscured are denoted obscured (obs.). Carbon-13 NMR spectra were recorded at 75 or $100 \mathrm{MHz}$ using $\mathrm{CDCl}_{3}\left(\delta_{\mathrm{C}} 77.16\right)$ or $\left(\mathrm{CD}_{3}\right)_{2} \mathrm{SO}\left(\delta_{\mathrm{C}} 39.52\right)$ as internal reference. Fluorine-19 NMR spectra were recorded at 377 $\mathrm{MHz}$ using $\mathrm{C}_{6} \mathrm{~F}_{6}\left(\delta_{\mathrm{F}}-164.92\right)$ as external reference.

Optical rotations were recorded on an Atago AP-100 automatic polarimeter at $589 \mathrm{~nm}$ and reported as follows: $[\alpha]_{\mathrm{D}}^{25}$, concentration ( $c$ in $\mathrm{g} / 100 \mathrm{~mL}$ ), and solvent. Melting points were recorded on a Stuart Scientific Analogue SMP11. Infrared spectra were recorded on a Brucker Alpha Spectrometer (ATR). High-resolution mass-spectra were obtained on a Waters QTogf API US, a Thermo Finnigan MAT 95XP spectrometer or on a on a Waters XevoQTof spectrometer. 


\section{Experimental Procedures and Characterization Data: \\ Unreported Starting Materials}

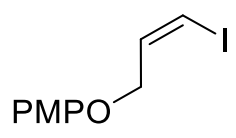

91

(Z)-1-(4-Methoxy)phenyloxy-3-iodoprop-2-ene 9l. To a solution of 4-methoxyphenol (372 mg, $3.0 \mathrm{mmol}$ ), (Z)-3-iodoprop-2-en-1-ol ${ }^{\mathrm{S1}}(662 \mathrm{mg}, 3.6 \mathrm{mmol})$ and triphenylphosphine $(944 \mathrm{mg}, 3.6$ $\mathrm{mmol})$ in THF $(6 \mathrm{~mL})$ was added dropwise di-iso-propylazodicarboxylate $(710 \mu \mathrm{L}, 3.6 \mathrm{mmol})$ at $0{ }^{\circ} \mathrm{C}$. The resulting yellow reaction mixture was stirred at $0{ }^{\circ} \mathrm{C}$ for 30 minutes, then warmed to rt and concentrated under reduced pressure. The crude residue was finally purified by flash column chromatography over silica gel (cyclohexane/EtOAc: 90/10) to yield the desired iodoalkene $9 \mathrm{l}$ as a yellow oil (730 mg, $\left.2.8 \mathrm{mmol}, 84 \%) .{ }^{1} \mathrm{H} \mathrm{NMR} \mathrm{(300} \mathrm{MHz,} \mathrm{CDCl}_{3}\right): \delta 6.85(\mathrm{~s}, 4 \mathrm{H})$, $6.59(\mathrm{dt}, J=7.9 \mathrm{~Hz}$ and $5.2 \mathrm{~Hz}, 1 \mathrm{H}), 6.47(\mathrm{dt}, J=7.9 \mathrm{~Hz}$ and $1.8 \mathrm{~Hz}, 1 \mathrm{H}), 4.58(\mathrm{dd}, J=5.2 \mathrm{~Hz}$ and $1.8 \mathrm{~Hz}, 2 \mathrm{H}), 3.77(\mathrm{~s}, 3 \mathrm{H}) ;{ }^{13} \mathrm{C}$ NMR $\left(75 \mathrm{MHz}, \mathrm{CDCl}_{3}\right): \delta 154.3,152.4,137.7,115.9,114.8,83.4$, 71.7, 55.9; IR (ATR): $v_{\max } 2831,2852,1505,1461,1284,1226,1039,822,753,644 \mathrm{~cm}^{-1}$; EIHRMS $\mathrm{m} / z$ calcd for $\mathrm{C}_{10} \mathrm{H}_{11} \mathrm{IO}_{2}[\mathrm{M}]^{+} 289.9798$, found 289.9801 .

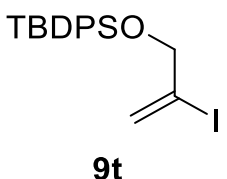

1-tert-Butyldiphenylsilyloxy-2-iodo-prop-2-ene 9t. To a solution of 2-iodoprop-2-en-1-ols2 (960 $\mathrm{mg}, \quad 5.2 \mathrm{mmol})$ in dichloromethane $(3 \mathrm{~mL})$ were successively added tert-butyldiphenylchlorosilane $(1.5 \mathrm{~mL}, 5.7 \mathrm{mmol})$ and imidazole $(388 \mathrm{mg}, 5.7 \mathrm{mmol})$. The resulting reaction mixture was stirred at $\mathrm{rt}$ for $14 \mathrm{~h}$, and diluted with diethyl ether. The organic

\footnotetext{
S1 This compound was prepared in two steps from ethyl propiolate according to: a) D. Beruben, I. Marek, J.-F. Normant, N. Platzer, J. Org. Chem. 1995, 60, 2488. b) I. Marek, C. Meyer, J.-F. Normant, Org. Synth. $1997,74,194$.

s2 This compound was prepared according to: N. Kamiya, Y. Chikami, Y. Ishii, Synlett 1990, 675.
} 
layer was washed twice with brine, dried over anhydrous magnesium sulfate, filtered and concentrated. The crude residue was finally purified by flash column chromatography over silica gel (cyclohexane/EtOAc: 95/5) to yield the desired protected alcohol 9t as a colorless oil (1.7g, 4.0 mmol, 78\%). ${ }^{1} \mathrm{H}$ NMR (300 MHz, $\left.\mathrm{CDCl}_{3}\right): \delta 7.67$ (d, J = 7.8 Hz, 4H), 7.45-7.31 (m, $\left.6 \mathrm{H}\right), 6.55$ (d, $J=1.5 \mathrm{~Hz}, 1 \mathrm{H}), 5.85(\mathrm{~d}, J=1.2 \mathrm{~Hz}, 1 \mathrm{H}), 4.20(\mathrm{t}, J=1.8 \mathrm{~Hz}, 2 \mathrm{H}), 1.08(\mathrm{~s}, 9 \mathrm{H}) ;{ }^{13} \mathrm{C} \mathrm{NMR}(75 \mathrm{MHz}$, $\left.\mathrm{CDCl}_{3}\right): \delta 135.6,133.1,130.0,127.9,123.4,108.9,71.5,26.9,19.4 ; \mathrm{IR}$ (ATR): $v_{\max } 2930,2856$, 1625, 1471, 1427, 1391, 1361, 1111, 1078, 899, 823, 739, $699 \mathrm{~cm}^{-1}$; EIHRMS m/z calcd for $\mathrm{C}_{19} \mathrm{H}_{23}$ IOSi $[\mathrm{M}]^{+} 422.0557$, found 422.0568 .

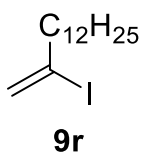

2-lodotetradec-1-ene 9r. To a suspension of 1,3-bis(diphenylphosphino)propane nickel(II) chloride (114 mg, $0.2 \mathrm{mmol})$ in THF $(7 \mathrm{~mL})$ was added dropwise diisobutylaluminium hydride (1.0 M solution in hexane, $9.1 \mathrm{~mL}, 9.1 \mathrm{mmol}$ ) at $\mathrm{rt}$. The resulting reaction mixture was cooled to $0^{\circ} \mathrm{C}$ before adding dropwise tetradec-1-yne $(1.7 \mathrm{~mL}, 7.0 \mathrm{mmol})$. The resulting solution was allowed to warm to $\mathrm{rt}$ and stirred for $2 \mathrm{~h}$. A solution of $\mathrm{N}$-iodosuccinimide $(3.2 \mathrm{~g}, 14.0 \mathrm{mmol})$ in THF $(21 \mathrm{~mL})$ was then added via a cannula at $0^{\circ} \mathrm{C}$ and the reaction was further stirred at $\mathrm{rt}$ for $2 \mathrm{~h}$. The reaction mixture was then quenched with a saturated aqueous solution of sodium potassium tartrate and the mixture was stirred at rt until complete dissolution of the aluminium salts. The aqueous layer was then extracted with diethyl ether and the combined organic layers were dried over anhydrous magnesium sulfate, filtered and concentrated. The crude residue was finally purified by flash column chromatography over silica gel (cyclohexane) to yield the desired iodoalkene $9 \mathrm{r}$ as a yellow oil (923 mg, $2.9 \mathrm{mmol}, 41 \%) .{ }^{1} \mathrm{H} \mathrm{NMR} \mathrm{(300} \mathrm{MHz,} \mathrm{CDCl}_{3}$ ): $\delta 6.00$ (d, $J=1.5 \mathrm{~Hz}, 1 \mathrm{H}), 5.68(\mathrm{~d}, J=1.2 \mathrm{~Hz}, 1 \mathrm{H}), 2.37(\mathrm{t}, J=6.9 \mathrm{~Hz}, 2 \mathrm{H}), 1.54-1.44(\mathrm{~m}, 2 \mathrm{H}), 1.33-1.17$ $(\mathrm{m}, 18 \mathrm{H}), 0.88(\mathrm{t}, J=6.9 \mathrm{~Hz}, 3 \mathrm{H}) ;{ }^{13} \mathrm{C} \mathrm{NMR}\left(75 \mathrm{MHz}, \mathrm{CDCl}_{3}\right): \delta 125.2,113.0,45.5,32.1,29.8(3 \mathrm{C})$, 29.7, 29.5 (2C), 29.2, 28.3, 22.8, 14.3; IR (ATR): $v_{\max }$ 2923, 2852, 1616, 1464, 1377, 1154, 1101, $889,721,619 \mathrm{~cm}^{-1}$; ESHRMS $\mathrm{m} / \mathrm{z}$ calcd for $\mathrm{C}_{14} \mathrm{H}_{27} \mathrm{l}[\mathrm{M}]^{+} 322.1152$, found 322.1160 . 


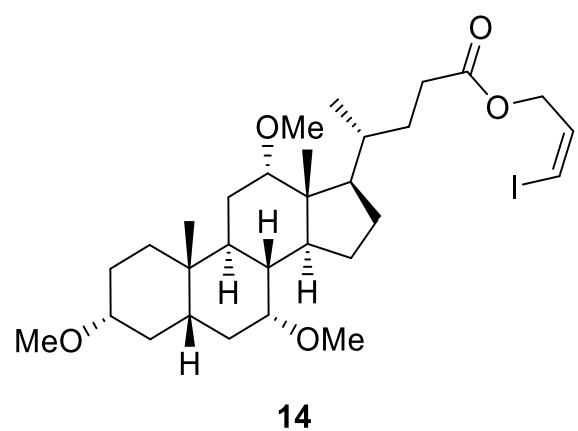

(Z)-3-lodoallyl $(R)-4-[(3 R, 5 S, 7 R, 8 R, 9 S, 10 S, 12 S, 13 R, 14 S, \quad 17 R)-3, \quad 7$, 12-trimethoxy-10, 13dimethylhexadecahydro-1H-cyclopenta[a]phenanthren-17-yl]pentanoate 14 . To a solution of $(R)-4-[(3 R, 5 S, 7 R, 8 R, 9 S, 10 S, 12 S, 13 R, 14 S, 17 R)-3,7$, 12-trimethoxy-10, 13-dimethylhexadecahydro-1H-cyclopenta[a]phenanthren-17-yl]pentanoic $\quad$ acid $^{\mathrm{S3}} \quad(600 \quad \mathrm{mg}, \quad 1.3 \quad \mathrm{mmol})$, 4(dimethylamino)pyridine (145 mg, $1.2 \mathrm{mmol}$ ) and (Z)-3-iodoprop-2-en-1-ol (724 mg, $4.0 \mathrm{mmol}$ ) in dichloromethane $(1.3 \mathrm{~mL})$ was added dicyclohexylcarbodiimide $(272 \mathrm{mg}, 1.3 \mathrm{mmol})$ at $0^{\circ} \mathrm{C}$. The resulting reaction mixture was allowed to warm to $\mathrm{rt}$ and stirred for $48 \mathrm{~h}$. The reaction mixture was then filtered and the filtrate was successively washed twice with a $0.5 \mathrm{M}$ aqueous solution of hydrochloric acid and a saturated aqueous solution of sodium bicarbonate. The organic layer was dried over anhydrous magnesium sulfate, filtered and concentrated. The crude residue was finally purified by flash column chromatography over silica gel (petroleum ether/ethyl acetate: 80/20) to yield the desired iodoalkene 14 as a pale yellow solid (518 mg, $8.5 \mathrm{mmol}, 64 \%) ; \mathrm{Mp}: 69^{\circ} \mathrm{C} ;[\alpha]_{\mathrm{D}}^{25}+26.5\left(c\right.$ 1.0, $\left.\mathrm{CHCl}_{3}\right) ;{ }^{1} \mathrm{H} \mathrm{NMR}\left(300 \mathrm{MHz}, \mathrm{CDCl}_{3}\right): \delta 6.53-6.40(\mathrm{~m}$, $2 \mathrm{H}), 4.64(\mathrm{~d}, J=4.8 \mathrm{~Hz}, 2 \mathrm{H}), 3.38-3.34(\mathrm{~m}, 1 \mathrm{H}), 3.32(\mathrm{~s}, 3 \mathrm{H}), 3.25(\mathrm{~s}, 3 \mathrm{H}), 3.20(\mathrm{~s}, 3 \mathrm{H}), 3.13(\mathrm{~d}, J=$ $2.1 \mathrm{~Hz}, 1 \mathrm{H}), 3.00-2.93(\mathrm{~m}, 1 \mathrm{H}), 2.45-2.33(\mathrm{~m}, 1 \mathrm{H}), 2.30-1.99(\mathrm{~m}, 4 \mathrm{H}), 1.96-1.67(\mathrm{~m}, 8 \mathrm{H}), 1.62-1.15$ $(\mathrm{m}, 10 \mathrm{H}), 1.09-0.95(\mathrm{~m}, 1 \mathrm{H}), 0.89$ (br. s, 6H), $0.64(\mathrm{~s}, 3 \mathrm{H}) ;{ }^{13} \mathrm{C} \mathrm{NMR}\left(75 \mathrm{MHz}, \mathrm{CDCl}_{3}\right): \delta 174.2$, 136.0, 84.8, 82.1, 80.9, 77.1, 66.9, 56.0, 55.8, 55.5, 46.3, 46.2, 42.8, 42.1, 39.8, 35.4, 35.2, 35.1, 34.6, 31.1, 31.0, 28.1, 27.9, 27.5, 26.9, 23.3, 23.0, 22.1, 17.5, 12.6; IR (ATR): $v_{\max } 2938,1743$, $1450,1371,1278,1222,1170,1096,658 \mathrm{~cm}^{-1}$; ESIHRMS $\mathrm{m} / \mathrm{z}$ calcd for $\mathrm{C}_{30} \mathrm{H}_{50} \mathrm{OO}_{5}[\mathrm{M}+\mathrm{H}]^{+}$ 617.2697, found 617.2701.

\footnotetext{
S3 Prepared from cholic acid according to: M. Rueda-Becerril, C. Chatalora, J. Leu, T. Okbinoglu, P. Kennepohl, J.-F. Paquin, G. Sammis, J. Am. Chem. Soc. 2012, 134, 4026.
} 


\section{Experimental Procedures and Characterization Data: Copper-Catalyzed I/Br $\rightarrow \mathrm{Cl}$ Exchange from Alkenyl lodides/Bromides}

\section{General procedure}

An oven dried $15 \mathrm{~mL}$ resealable pressure tube was charged with copper iodide (19 $\mathrm{mg}, 0.1$ $\mathrm{mmol})$, tetramethylammonium chloride $(219 \mathrm{mg}, 2.0 \mathrm{mmol})$ and the iodoalkene or bromoalkene (1.0 mmol). The tube was fitted with a rubber septum, evacuated under vacuum, backfilled with argon and trans- $N, N^{\prime}$-dimethylcyclohexane-1,2-diamine (31 $\mu \mathrm{L}, 0.2 \mathrm{mmol}$ ) and ethanol (or DMSO when specified) $(2 \mathrm{~mL}$ ) were next added. The tube was closed with a Teflon-coated screw cap and the resulting suspension was stirred and heated at $110^{\circ} \mathrm{C}$ in a preheated oil bath for $48 \mathrm{~h}$ (unless specified otherwise). When the reaction was run in ethanol, the crude reaction mixture was cooled to rt, diluted with ethyl acetate, filtered on a plug of silica gel and concentrated. When the reaction was run in DMSO, the crude reaction mixture was cooled to rt, diluted with water $(15 \mathrm{~mL})$, extracted thrice with diethyl ether and the combined organic layers were dried over anhydrous magnesium sulfate, filtered and concentrated. The crude residue was finally purified by flash column chromatography over silica gel to afford the desired chloroalkene.

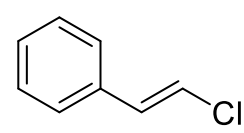

$10 \mathrm{a}$

(E)-(2-Chlorovinyl)benzene 10a. Yield (from the corresponding iodoalkene): $82 \%$ (113 $\mathrm{mg}, 0.82$ $\mathrm{mmol})$. Solvent system for flash chromatography: pentane. Colorless oil. This compound has been previously reported. ${ }^{\mathrm{S4}}$

\footnotetext{
S4 J. Bull, J. Mousseau, A. Charrette, Org. Lett. 2008, 10, 5485.
} 


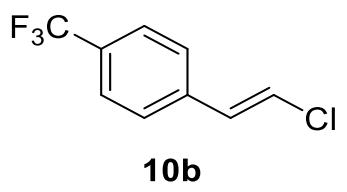

(E)-1-(2-Chlorovinyl)-4-(trifluoromethyl)benzene 10b. Yield (from the corresponding iodoalkene in DMSO for 24h): $71 \%$ (115 mg, $0.58 \mathrm{mmol}$ ); Yield (from the corresponding bromoalkene): $74 \%$ (31 mg, $0.14 \mathrm{mmol}$ ). Solvent system for flash chromatography: pentane; Yellow oil; ${ }^{1} \mathrm{H}$ NMR (300 $\left.\mathrm{MHz}, \mathrm{CDCl}_{3}\right): \delta 7.58(\mathrm{~d}, J=8.1 \mathrm{~Hz}, 2 \mathrm{H}), 7.40(\mathrm{~d}, J=8.1 \mathrm{~Hz}, 2 \mathrm{H}), 6.86(\mathrm{~d}, J=13.5 \mathrm{~Hz}, 1 \mathrm{H}), 6.75(\mathrm{~d}, J$ $=13.5 \mathrm{~Hz}, 1 \mathrm{H}) ;{ }^{13} \mathrm{C}$ NMR $\left(100 \mathrm{MHz}, \mathrm{CDCl}_{3}\right): \delta 138,4,132.2,130.1$ (q, $\left.J=32.3 \mathrm{~Hz}\right), 126.4,125.9$ (q, $J=3.8 \mathrm{~Hz}), 124.1(\mathrm{q}, J=270.4 \mathrm{~Hz}), 121.6 ;{ }^{19} \mathrm{~F} \mathrm{NMR}\left(377 \mathrm{MHz}, \mathrm{CDCl}_{3}\right): \delta-65.8(\mathrm{~s}) ; \mathrm{IR}$ (ATR): $v_{\max }$ $3074,1614,1412,1324,1165,1123,1109,1067,1017,931,845,823,795,755,728 \mathrm{~cm}^{-1}$; EIHRMS $\mathrm{m} / \mathrm{z}$ calcd for $\mathrm{C}_{9} \mathrm{H}_{6}{ }^{35} \mathrm{ClF}_{3}[\mathrm{M}]^{+}$206.0105, found 206.0106 .

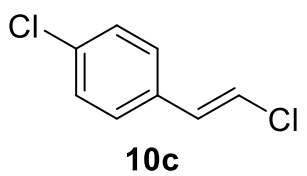

(E)-1-(2-Chlorovinyl)-4-chlorobenzene 10c. Yield (from the corresponding iodoalkene): $67 \%$ (116 mg, $0.67 \mathrm{mmol}$ ); Yield (from the corresponding bromoalkene): Yield: 77\% (27 mg, 0.15 mmol). Solvent system for flash chromatography: pentane; White solid; Mp: $33^{\circ} \mathrm{C} ;{ }^{1} \mathrm{H}$ NMR (300 $\left.\mathrm{MHz}, \mathrm{CDCl}_{3}\right): \delta 7.29(\mathrm{~d}, J=8.4 \mathrm{~Hz}, 2 \mathrm{H}), 7.21(\mathrm{~d}, J=8.4 \mathrm{~Hz}, 2 \mathrm{H}), 6.77(\mathrm{~d}, J=13.8 \mathrm{~Hz}, 1 \mathrm{H}), 6.62(\mathrm{~d}, J$ $=13.8 \mathrm{~Hz}, 1 \mathrm{H}) ;{ }^{13} \mathrm{C}$ NMR $\left(75 \mathrm{MHz}, \mathrm{CDCl}_{3}\right): \delta 134.0,133.2,132.2,129.1,127.4,119.5$; IR (ATR): $V_{\max } 1488,1403,1090,1012,944,927,832,818 \mathrm{~cm}^{-1}$; ElHRMS $\mathrm{m} / \mathrm{z}$ calcd for $\mathrm{C}_{8} \mathrm{H}_{6}{ }^{35} \mathrm{Cl}_{2}[\mathrm{M}]^{+}$ 171.9841, found 171.9851 . 


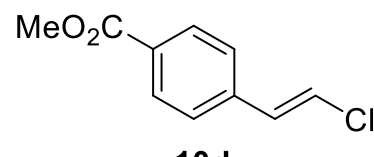

(E)-Methyl-4-(2-chlorovinyl)benzoate 10d. Yield (from the corresponding iodoalkene in DMSO for $24 \mathrm{~h}$ ): $79 \%$ (155 $\mathrm{mg}, 0.79 \mathrm{mmol}$ ). Solvent system for flash chromatography: petroleum ether/EtOAc: 98/2. This compound has been previously reported. ${ }^{55}$

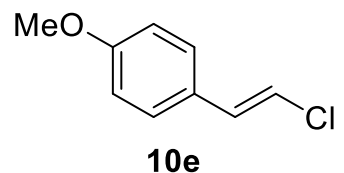

(E)-(2-Chlorovinyl)-4-methoxybenzene 10e. Yield (from the corresponding iodoalkene): 50\% (84 $\mathrm{mg}, 0.50 \mathrm{mmol}$ ). Solvent system for flash chromatography: cyclohexane/EtOAc: 95/5. This compound has been previously reported. ${ }^{\mathrm{s} 6}$

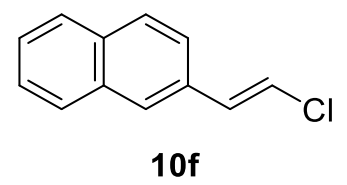

(E)-2-(2-Chlorovinyl)naphthalene 10f. Yield (from the corresponding iodoalkene in DMSO for 24h): $80 \%$ (151 $\mathrm{mg}, 0.80 \mathrm{mmol})$. Solvent system for flash chromatography: pentane. This compound has been previously reported. ${ }^{55}$

${ }_{55}^{5}$ T. Matsuda, K. Suzuki, N. Miura, Adv. Synth. Catal. 2013, 355, 3396.

${ }^{56}$ J. Barluenga, P. Moriel, F. Aznar, C. Valdès, Adv. Synth. Catal. 2006, 348, 347. 


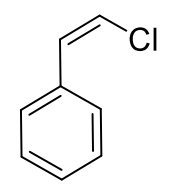

$10 \mathrm{~g}$

(Z)-(2-Chlorovinyl)benzene 10g. Yield (from the corresponding iodoalkene): 83\% (115 mg, 0.83 $\mathrm{mmol}$ ); Yield (from the corresponding bromoalkene): 75\% (21 mg, $0.15 \mathrm{mmol}$ ). Solvent system for flash chromatography: pentane; Colorless oil; ${ }^{1} \mathrm{H}$ NMR $\left(300 \mathrm{MHz}, \mathrm{CDCl}_{3}\right): \delta 7.67(\mathrm{~d}, J=7.2 \mathrm{~Hz}$, $2 \mathrm{H}), 7.41-7.29(\mathrm{~m}, 3 \mathrm{H}), 6.64(\mathrm{~d}, J=8.1 \mathrm{~Hz}, 1 \mathrm{H}), 6.27(\mathrm{~d}, J=8.1 \mathrm{~Hz}, 1 \mathrm{H}) ;{ }^{13} \mathrm{C} \mathrm{NMR}\left(75 \mathrm{MHz}, \mathrm{CDCl}_{3}\right)$ : $\delta 134.3,129.4,129.3,128.4,128.3,117.7$; IR (ATR): $v_{\max } 3025,1617,1491,1445,1346,926,846$, $772,722,689,658 \mathrm{~cm}^{-1}$; ElHRMS $\mathrm{m} / z$ calcd for $\mathrm{C}_{8} \mathrm{H}_{7}{ }^{35} \mathrm{Cl}[\mathrm{M}]^{+} 138.0231$, found 138.0239 .

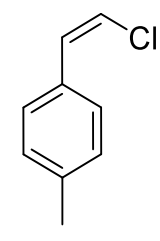

$10 \mathrm{~h}$

(Z)-(2-Chlorovinyl)-4-methylbenzene 10h. Yield (from the corresponding iodoalkene): 81\% (115 $\mathrm{mg}, 0.81 \mathrm{mmol}$ ). Solvent system for flash chromatography: pentane; Colorless oil; ${ }^{1} \mathrm{H}$ NMR (300 $\left.\mathrm{MHz}, \mathrm{CDCl}_{3}\right): \delta 7.57(\mathrm{~d}, J=8.1 \mathrm{~Hz}, 2 \mathrm{H}), 7.18(\mathrm{~d}, J=8.1 \mathrm{~Hz}, 2 \mathrm{H}), 6.60(\mathrm{~d}, J=8.1 \mathrm{~Hz}, 1 \mathrm{H}), 6.21(\mathrm{~d}, J=$ $8.1 \mathrm{~Hz}, 1 \mathrm{H}), 2.36$ (s, 3H); ${ }^{13} \mathrm{C}$ NMR $\left(75 \mathrm{MHz}^{\mathrm{CDCl}} 3\right): \delta 138.3,131.5,129.3,129.3,129.1,116.8$, 21.5; IR (ATR): $V_{\max } 2922,1615,1509,1448,1344,1173,1120,856,821,786,730,694,621 \mathrm{~cm}^{-1}$; EIHRMS $m / z$ calcd for $\mathrm{C}_{9} \mathrm{H}_{9}{ }^{35} \mathrm{Cl}[\mathrm{M}]^{+} 152.0387$, found 152.0393 .

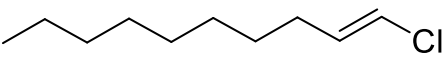

$10 \mathbf{i}$

(E)-1-Chlorodec-1-ene 10i. Yield (from the corresponding iodoalkene): $77 \%$ (134 $\mathrm{mg}, 0.77$ $\mathrm{mmol})$; Yield (from the corresponding bromoalkene): 84 \% (29 mg, $0.16 \mathrm{mmol}$ ). Solvent system 
for flash chromatography: pentane; Colorless oil; ${ }^{1} \mathrm{H}$ NMR (300 MHz, $\left.\mathrm{CDCl}_{3}\right): \delta 5.96-5.84(\mathrm{~m}, 2 \mathrm{H})$, 2.04 (app. qd, $J=7.2 \mathrm{~Hz}$ and $1.2 \mathrm{~Hz}, 2 \mathrm{H}), 1.41-1.20(\mathrm{~m}, 12 \mathrm{H}), 0.88(\mathrm{t}, J=6.9 \mathrm{~Hz}, 3 \mathrm{H}) ;{ }^{13} \mathrm{C} \mathrm{NMR}(75$ $\left.\mathrm{MHz} \mathrm{CDCl}_{3}\right): \delta 134.2,116.7,32.0,31.0,29.5,29.4,29.1,29.0,22.8,14.2 ;$ IR (ATR): $v_{\max } 2956$, 2925, 2855, 1457, 1377, 931, 805, $721 \mathrm{~cm}^{-1}$; ElHRMS $\mathrm{m} / \mathrm{z}$ calcd for $\mathrm{C}_{10} \mathrm{H}_{19}{ }^{35} \mathrm{Cl}[\mathrm{M}]^{+} 174.1170$, found 174.1178 .

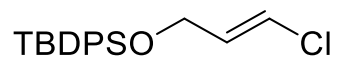

$10 \mathrm{j}$

(E)-1-tert-Butyldiphenylsilyloxy-3-chloroprop-2-ene 10j. Yield (from the corresponding iodoalkene - reaction performed on a $0.5 \mathrm{mmol}$ scale): $92 \%$ (151 $\mathrm{mg}, 0.46 \mathrm{mmol})$. Solvent system for flash chromatography: cyclohexane/EtOAc: 98/2; Colorless oil; ${ }^{1} \mathrm{H}$ NMR (300 MHz, $\left.\mathrm{CDCl}_{3}\right): \delta 7.71(\mathrm{~d}, J=7.5 \mathrm{~Hz}, 4 \mathrm{H}), 7.50-7.38(\mathrm{~m}, 6 \mathrm{H}), 6.27(\mathrm{dt}, J=12.9 \mathrm{~Hz}$ and $1.8 \mathrm{~Hz}, 1 \mathrm{H}), 6.03(\mathrm{dt}$, $J=13.2 \mathrm{~Hz}$ and $4.8 \mathrm{~Hz}, 1 \mathrm{H}), 4.20(\mathrm{dd}, J=4.8 \mathrm{~Hz}$ and $1.5 \mathrm{~Hz}, 2 \mathrm{H}), 1.1(\mathrm{~s}, 9 \mathrm{H}) ;{ }^{13} \mathrm{C} \mathrm{NMR}(75 \mathrm{MHz}$, $\left.\mathrm{CDCl}_{3}\right): \delta 135.6,133.3,132.3,129.9,127.9,119.0,62.7,26.9,19.3 ;$ IR (ATR): $V_{\max } 2930,2857$, 1471, 1376, 1111, 1067, 1007, 962, 926, 822, 767, 738, 700, $614 \mathrm{~cm}^{-1}$; ElHRMS m/z calcd for $\mathrm{C}_{19} \mathrm{H}_{23}{ }^{35} \mathrm{ClOSi}[\mathrm{M}]^{+} 330.1201$, found 330.1205.

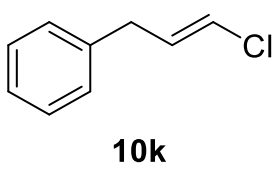

(E)-(3-Chloroallyl)benzene 10k. Yield (from the corresponding iodoalkene): 82\% (125 mg, 0.82 mmol). Solvent system for flash chromatography: pentane; Orange oil; ${ }^{1} \mathrm{H}$ NMR (300 MHz, $\left.\mathrm{CDCl}_{3}\right): \delta$ 7.37-7.12 (m, 5H), 6.15-5.95 (m, 2H), $3.39(\mathrm{~d}, J=6.3 \mathrm{~Hz}, 2 \mathrm{H}) ;{ }^{13} \mathrm{C} \mathrm{NMR}\left(75 \mathrm{MHz}, \mathrm{CDCl}_{3}\right)$ : $\delta$ 138.7, 132.7, 128.8, 128.6, 126.7, 118.5, 37.2; IR (ATR): $v_{\max } 3028,1702,1632,1495,1453$, 1431, 1286, 1241, 1124, 943, 779, 747, $697 \mathrm{~cm}^{-1}$; ElHRMS m/z calcd for $\mathrm{C}_{9} \mathrm{H}_{9}{ }^{35} \mathrm{Cl}[\mathrm{M}]^{+} 152.0387$, found 152.0391. 


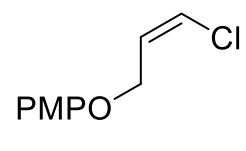

101

(Z)-3-Chloro-1-(4-methoxy)phenyloxy-prop-2-ene 10l. Yield (from the corresponding iodoalkene - reaction performed on a $0.5 \mathrm{mmol}$ scale using 20 mol\% of copper iodide and 40 mol\% of trans- $N, N^{\prime}$-dimethylcyclohexane-1,2-diamine in DMSO): $64 \%$ (64 mg, $0.32 \mathrm{mmol}$ ). Solvent system for flash chromatography: pentane/Et $2 \mathrm{O}: 98 / 2$; Colorless oil; ${ }^{1} \mathrm{H} \mathrm{NMR}$ (300 MHz, $\left.\mathrm{CDCl}_{3}\right): \delta 6.85(\mathrm{~s}, 4 \mathrm{H}), 6.23(\mathrm{dt}, J=7.2 \mathrm{~Hz}$ and $1.8 \mathrm{~Hz}, 1 \mathrm{H}), 6.05(\mathrm{dt}, J=7.2 \mathrm{~Hz}$ and $1.5 \mathrm{~Hz}, 1 \mathrm{H})$, $4.72(\mathrm{dd}, J=5.7 \mathrm{~Hz}$ and $1.8 \mathrm{~Hz}, 2 \mathrm{H}), 3.77(\mathrm{~s}, 3 \mathrm{H}) ;{ }^{13} \mathrm{C} \mathrm{NMR}\left(75 \mathrm{MHz}, \mathrm{CDCl}_{3}\right): \delta 154.3,152.4,128.4$, 120.7, 115.8, 114.8, 64.4, 55.7; IR (ATR): $V_{\max } 2833,1506,1462,1227,1181,1107,1039,823$, 751, $700 \mathrm{~cm}^{-1}$; EIHRMS $\mathrm{m} / z$ calcd for $\mathrm{C}_{10} \mathrm{H}_{11}{ }^{35} \mathrm{ClO}_{2}[\mathrm{M}]^{+}$198.0442, found 198.0452 .

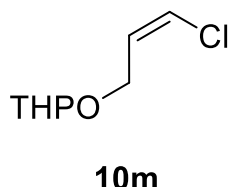

(Z)-3-Chloro-1-(2-Tetrahydro-2H-pyranyloxy)-prop-2-ene $10 \mathrm{~m}$. Yield (from the corresponding iodoalkene): $69 \%$ (122 $\mathrm{mg}, 0.69 \mathrm{mmol})$. Solvent system for flash chromatography: pentane/Et ${ }_{2} \mathrm{O}$ : 80/20; Brown oil; ; ${ }^{1} \mathrm{H}$ NMR (300 MHz, $\mathrm{CDCl}_{3}$ ): $\delta 6.15$ (dt, J $=7.2 \mathrm{~Hz}$ and $1.8 \mathrm{~Hz}$, 1H), 5.98 (app. dd, $J=12.9 \mathrm{~Hz}$ and $6.3 \mathrm{~Hz}, 1 \mathrm{H}), 4.65(\mathrm{t}, J=3.6 \mathrm{~Hz}, 1 \mathrm{H}), 4.41$ (A of ABXY syst., $J=$ 13.3 Hz and $5.6 \mathrm{~Hz}$ and $1.7 \mathrm{~Hz}, 1 \mathrm{H}$ ), 4.26 (B of $A B X Y$ syst., $J=13.3 \mathrm{~Hz}$ and $6.4 \mathrm{~Hz}$ and $1.6 \mathrm{~Hz}, 1 \mathrm{H}$ ), $3.88(\mathrm{~m}, 1 \mathrm{H}), 3.53(\mathrm{~m}, 1 \mathrm{H}), 1.88-1.47(\mathrm{~m}, 6 \mathrm{H}) ;{ }^{13} \mathrm{C}$ NMR $\left(75 \mathrm{MHz}, \mathrm{CDCl}_{3}\right): \delta$ 129.0, 120.1, 98.7, $62.9,62.5,30.7,25.5,19.6$; IR (ATR): $v_{\max } 2942,1634,1454,1351,1201,1156,1120,1070,1029$, 964, 906, 870, 815, 733, $683 \mathrm{~cm}^{-1}$; ESIHRMS $\mathrm{m} / \mathrm{z}$ calcd for $\mathrm{C}_{8} \mathrm{H}_{13}{ }^{35} \mathrm{ClO}_{2}[\mathrm{M}]^{+}$176.0599, found 176.0609. 


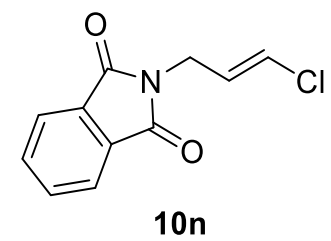

(E)-3-Chloro-1-phthalimido-prop-2-ene 10n. Yield (from the corresponding iodoalkene reaction performed on a $0.5 \mathrm{mmol}$ scale): $91 \%$ (101 mg, $0.45 \mathrm{mmol})$. Solvent system for flash chromatography: petroleum ether/ethyl acetate: 80/20; White solid; Mp: $102^{\circ} \mathrm{C} ;{ }^{1} \mathrm{H}$ NMR (300 $\left.\mathrm{MHz}, \mathrm{CDCl}_{3}\right): \delta 7.85(\mathrm{dd}, J=5.4 \mathrm{~Hz}$ and $3 \mathrm{~Hz}, 2 \mathrm{H}), 7.72(\mathrm{dd}, J=5.7 \mathrm{~Hz}$ and $3 \mathrm{~Hz}, 2 \mathrm{H}), 6.35(\mathrm{~d}, J=$ $13.5 \mathrm{~Hz}, 1 \mathrm{H}), 6.00(\mathrm{dt}, J=13.2 \mathrm{~Hz}$ and $7.2 \mathrm{~Hz}, 1 \mathrm{H}), 4.26(\mathrm{dd}, J=6.3 \mathrm{~Hz}$ and $0.6 \mathrm{~Hz}, 2 \mathrm{H}) ;{ }^{13} \mathrm{C} \mathrm{NMR}$ (75 MHz, $\mathrm{CDCl}_{3}$ ): $\delta 167.7,134.3,132.1,126.9,123.6,123.5,37.4$; IR (ATR): $v_{\max } 3078,1703$, $1429,1393,1345,1120,1044,941,798,717,614 \mathrm{~cm}^{-1}$; ESIHRMS $\mathrm{m} / \mathrm{z}$ calcd for $\mathrm{C}_{11} \mathrm{H}_{9}{ }^{35} \mathrm{CINO}_{2}$ $[\mathrm{M}+\mathrm{H}]^{+} 222.0316$, found 222.0318 .

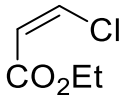

100

(Z)-Ethyl-4-chlorobut-3-enoate 10o. This compound is extremely volatile. NMR Yield using DMF as an internal standard (from the corresponding iodoalkene): $70 \%$. This compound has been previously reported. ${ }^{\mathrm{ST}}$

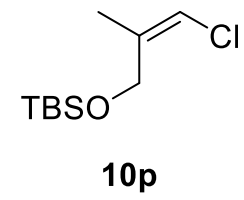

(Z)-1-tert-Butyldimethylsilyloxy-3-chloro-2-methyl-prop-2-ene 10p. Yield (from the corresponding iodoalkene): $88 \% \quad(196 \mathrm{mg}, 0.88 \mathrm{mmol})$. Solvent system for flash chromatography: pentane/Et $2 \mathrm{O}: 98 / 2$; Colorless oil; ${ }^{1} \mathrm{H} \mathrm{NMR}\left(300 \mathrm{MHz}, \mathrm{CDCl}_{3}\right): \delta 5.78(\mathrm{~s}, 1 \mathrm{H})$,

\footnotetext{
${ }^{7}$ M. R. Heinrich, O. Blank, D. Ullrich, M. Kirschstein, J. Org. Chem. 2007, 72, 9609.
} 
$4.33(\mathrm{~s}, 2 \mathrm{H}), 1.79(\mathrm{~s}, 3 \mathrm{H}), 0.90(\mathrm{~s}, 9 \mathrm{H}), 0.08(\mathrm{~s}, 6 \mathrm{H}) ;{ }^{13} \mathrm{C} \mathrm{NMR}\left(75 \mathrm{MHz}, \mathrm{CDCl}_{3}\right): \delta$ 138.5, 111.3, 61.7, 26.0 (2C), 18.5, -5.2; IR (ATR): $V_{\max } 2955,1471,1252,1088,836,775,667 \mathrm{~cm}^{-1}$; EIHRMS $m / z$ calcd for $\mathrm{C}_{10} \mathrm{H}_{21}{ }^{35} \mathrm{ClOSi}[\mathrm{M}]^{+} 220.1045$, found 220.1048 .

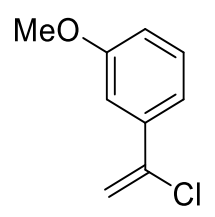

$10 q$

1-(1-Chlorovinyl)-3-methoxybenzene 10q. Yield (from the corresponding iodoalkene - reaction performed on a $0.5 \mathrm{mmol}$ scale using $20 \mathrm{~mol} \%$ of copper iodide and $40 \mathrm{~mol} \%$ of trans- $N, N^{\prime}$ dimethylcyclohexane-1,2-diamine in DMSO for $24 \mathrm{~h}$ ): $74 \%$ (63 mg, $0.37 \mathrm{mmol}$ ). Solvent system for flash chromatography: pentane; Colorless oil; ${ }^{1} \mathrm{H}$ NMR $\left(300 \mathrm{MHz}, \mathrm{CDCl}_{3}\right): \delta$ 7.31-7.15 (m, 3 H), 6.92-6.87 (m, 1H), $5.76(\mathrm{~d}, J=1.5 \mathrm{~Hz}, 1 \mathrm{H}), 5.52(\mathrm{~d}, J=1.8 \mathrm{~Hz}, 1 \mathrm{H}), 3.83(\mathrm{~s}, 3 \mathrm{H}) ;{ }^{13} \mathrm{C}$ NMR $(75$ $\mathrm{MHz}_{2} \mathrm{CDCl}_{3}$ ): $\delta 159.7,139.9,138.5,129.5,119.0,114.8,113.1,112.4,55.5 ;$ IR (ATR): $v_{\max } 2940$, $1692,1598,1580,1484,1464,1431,1320,1288,1261,1197,1167,1042,908,880,781,732$, $666 \mathrm{~cm}^{-1}$; EIHRMS $\mathrm{m} / \mathrm{z}$ calcd for $\mathrm{C}_{9} \mathrm{H}_{9}{ }^{35} \mathrm{ClO}[\mathrm{M}]^{+} 168.0336$, found 168.0337 .

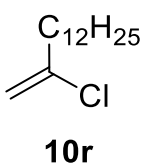

2-Chlorotetradec-1-ene 10r. Yield (from the corresponding iodoalkene - reaction performed on a $0.5 \mathrm{mmol}$ scale using $20 \mathrm{~mol} \%$ of copper iodide and $40 \mathrm{~mol} \%$ of trans- $N, N^{\prime}$ dimethylcyclohexane-1,2-diamine in DMSO for $24 \mathrm{~h}$ ): $77 \%$ (89 mg, $0.39 \mathrm{mmol}$ ). Solvent system for flash chromatography: pentane; Colorless oil; ${ }^{1} \mathrm{H}$ NMR $\left(300 \mathrm{MHz}, \mathrm{CDCl}_{3}\right): \delta 5.13$ (d, $J=0.9 \mathrm{~Hz}$, 1H), $5.10(\mathrm{~d}, J=1.2 \mathrm{~Hz}, 1 \mathrm{H}), 2.32(\mathrm{t}, J=7.5 \mathrm{~Hz}, 2 \mathrm{H}), 1.61-1.52(\mathrm{~m}, 2 \mathrm{H}), 1.33-1.21(\mathrm{~m}, 18 \mathrm{H}), 0.88(\mathrm{t}$, $J=6.9 \mathrm{~Hz}, 3 \mathrm{H}) ;{ }^{13} \mathrm{C}$ NMR $\left(75 \mathrm{MHz}, \mathrm{CDCl}_{3}\right): \delta 143.3,111.8,39.3,32.1,29.8(2 \mathrm{C}), 29.7,29.5(2 \mathrm{C})$, 
28.7, 27.3, 22.9, 14.3; IR (ATR): $v_{\max } 2923,2853,1634,1465,1173,908,876,735,640,617 \mathrm{~cm}^{-1}$; EIHRMS $m / z$ calcd for $\mathrm{C}_{14} \mathrm{H}_{27}{ }^{35} \mathrm{Cl}[\mathrm{M}]^{+} 230.1796$, found 230.1798 .

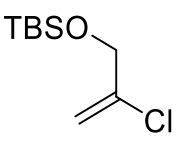

$10 \mathrm{~s}$

1-tert-Butyldimethylsilyloxy-2-chloro-prop-2-ene 10s. Yield (from the corresponding iodoalkene - reaction performed on a $0.5 \mathrm{mmol}$ scale using 20 mol\% of copper iodide and 40 mol\% of trans- $N, N^{\prime}$-dimethylcyclohexane-1,2-diamine in DMSO for $\left.24 \mathrm{~h}\right): 60 \%$ (61 $\mathrm{mg}, 0.30$ mmol). Solvent system for flash chromatography: pentane; Colorless oil; ${ }^{1} \mathrm{H}$ NMR (300 MHz, $\left.\mathrm{CDCl}_{3}\right): \delta 5.50(\mathrm{~d}, J=1.5 \mathrm{~Hz}, 1 \mathrm{H}), 5.28(\mathrm{~d}, J=1.2 \mathrm{~Hz}, 1 \mathrm{H}), 4.15(\mathrm{t}, J=1.5 \mathrm{~Hz}, 2 \mathrm{H}), 0.92(\mathrm{~s}, 9 \mathrm{H}), 0.1$ $(\mathrm{s}, 6 \mathrm{H}) ;{ }^{13} \mathrm{C}$ NMR $\left(75 \mathrm{MHz}, \mathrm{CDCl}_{3}\right): \delta 140.6,110.6,65.9,25.9,18.5,-5.2 ; \mathrm{IR}(\mathrm{ATR}): v_{\max } 2929,2857$, 1645, 1462, 1256, 1093, 884, 837, 777, $698 \mathrm{~cm}^{-1}$; ElHRMS $\mathrm{m} / \mathrm{z}$ calcd for $\mathrm{C}_{9} \mathrm{H}_{19}{ }^{35} \mathrm{ClOSi}[\mathrm{M}]^{+}$ 206.0888, found 206.0890 .

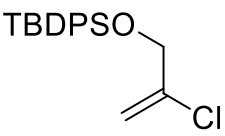

$10 \mathrm{t}$

1-tert-Butyldiphenylsilyloxy-2-chloro-prop-2-ene 10t. Yield (from the corresponding iodoalkene - reaction performed on a $0.5 \mathrm{mmol}$ scale using 20 mol\% of copper iodide and $40 \mathrm{~mol} \%$ of trans$N, N^{\prime}$-dimethylcyclohexane-1,2-diamine in DMSO for 24h): $70 \%$ (115 mg, $0.35 \mathrm{mmol}$ ); Yield (from the corresponding bromoalkene $(0.2 \mathrm{mmol})$ using $20 \mathrm{~mol} \%$ of copper iodide and $40 \mathrm{~mol} \%$ of trans- $N, N^{\prime}$-dimethylcyclohexane-1,2-diamine in DMSO for $24 \mathrm{~h}$ ): $85 \%$ (56 mg, $0.17 \mathrm{mmol}$ ). Solvent system for flash chromatography: pentane; Colorless oil; ${ }^{1} \mathrm{H} N M R\left(300 \mathrm{MHz}, \mathrm{CDCl}_{3}\right): \delta$ 7.71-7.64 (m, 4H), 7.49-7.36 (m, 6H), $5.66(\mathrm{~d}, J=1.5 \mathrm{~Hz}, 1 \mathrm{H}), 5.34(\mathrm{~d}, J=1.5 \mathrm{~Hz}, 1 \mathrm{H}), 4.18(\mathrm{t}, J=$ $1.5 \mathrm{~Hz}, 2 \mathrm{H}), 1.09$ (s, 9H); ${ }^{13} \mathrm{C}$ NMR (75 MHz, $\left.\mathrm{CDCl}_{3}\right): \delta 140.0,135.6,133.1,130.1,128.0,110.9$, 
66.3, 26.9, 19.4; IR (ATR): $V_{\max } 2931,2857,1647,1472,1427,1111,1089,885,824,738,700$, $613 \mathrm{~cm}^{-1}$; EIHRMS $\mathrm{m} / \mathrm{z}$ calcd for $\mathrm{C}_{19} \mathrm{H}_{23}{ }^{35} \mathrm{ClOSi}[\mathrm{M}]^{+} 330.1201$, found 330.1211 .

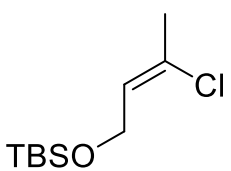

$10 u$

(Z)-1-tert-Butyldimethylsilyloxy-3-chloro-3-methyl-prop-2-ene 10u. Yield (from the corresponding iodoalkene - reaction performed on a $0.5 \mathrm{mmol}$ scale using $30 \mathrm{~mol} \%$ of copper iodide and 60 mol\% of trans- $N, N^{\prime}$-dimethylcyclohexane-1,2-diamine): $72 \%$ (80 mg, $0.36 \mathrm{mmol}$ ). Solvent system for flash chromatography: pentane/Et $2 \mathrm{O}: 98 / 2$; Colorless oil; ${ }^{1} \mathrm{H} N M R(300 \mathrm{MHz}$, $\left.\mathrm{CDCl}_{3}\right): \delta 5.60(\mathrm{t}, J=5.7 \mathrm{~Hz}, 1 \mathrm{H}), 4.30(\mathrm{dd}, J=5.7 \mathrm{~Hz}$ and $0.9 \mathrm{~Hz}, 2 \mathrm{H}), 2.10(\mathrm{~d}, J=0.9 \mathrm{~Hz}, 3 \mathrm{H}), 0.90$ (s, 9H), $0.08(\mathrm{~s}, 6 \mathrm{H}) ;{ }^{13} \mathrm{C}$ NMR $\left(75 \mathrm{MHz}, \mathrm{CDCl}_{3}\right): \delta$ 130.2, 126.1, 60.8, 26.0, 25.9, 18.3, -5.2; IR (ATR): $V_{\max } 2929,1471,1255,1101,1061,836,776,666 \mathrm{~cm}^{-1}$; ElHRMS $\mathrm{m} / \mathrm{z}$ calcd for $\mathrm{C}_{10} \mathrm{H}_{21}{ }^{35} \mathrm{ClOSi}[\mathrm{M}]^{+} 220.1045$, found 220.1047 .

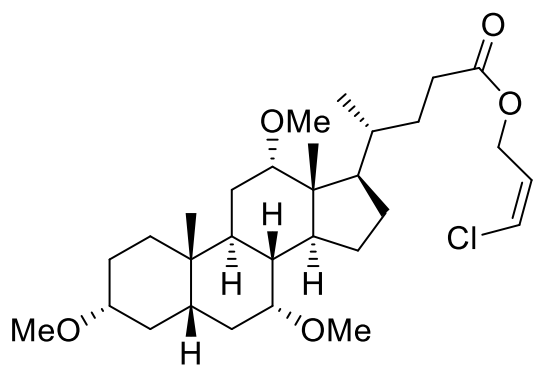

15

(Z)-3-Chloroallyl (R)-4-[(3R,5S,7R,8R,9S,10S,12S,13R,14S, 17R)-3, 7, 12-trimethoxy-10, 13dimethylhexadecahydro-1H-cyclopenta[a]phenanthren-17-yl]pentanoate 15 . Yield (from the corresponding iodoalkene - reaction performed on a $0.15 \mathrm{mmol}$ scale using $20 \mathrm{~mol} \%$ of copper iodide and 40 mol\% of trans- $N, N^{\prime}$-dimethylcyclohexane-1,2-diamine in DMSO for $96 \mathrm{~h}$ ): $48 \%$ (38 $\mathrm{mg}, 70 \mu \mathrm{mol})$. Solvent system for flash chromatography: petroleum ether/ethyl acetate: 80/20; 
Colorless oil; $[\alpha]_{\mathrm{D}}^{25}+34.0\left(c\right.$ 1.0, $\left.\mathrm{CHCl}_{3}\right) ;{ }^{1} \mathrm{H}$ NMR $\left(300 \mathrm{MHz}, \mathrm{CDCl}_{3}\right): \delta 6.21(\mathrm{dt}, J=7.5 \mathrm{~Hz}$ and 1.5 Hz, 1H), 5.93 (app. q, $J=7.2 \mathrm{~Hz}, 1 \mathrm{H}$ ), 4.76 (dd, $J=6 \mathrm{~Hz}$ and $1.5 \mathrm{~Hz}, 2 \mathrm{H}$ ), 3.35 (br. s, 1H), 3.33 (s, $3 \mathrm{H}), 3.25(\mathrm{~s}, 3 \mathrm{H}), 3.20(\mathrm{~s}, 3 \mathrm{H}), 3.15-3.10(\mathrm{~m}, 1 \mathrm{H}), 3.05-2.92(\mathrm{~m}, 1 \mathrm{H}), 2.38(\mathrm{~m}, 1 \mathrm{H}), 2.30-2.15(\mathrm{~m}$, $2 \mathrm{H}), 2.14-2.01(\mathrm{~m}, 2 \mathrm{H}), 1.95-1.68(\mathrm{~m}, 8 \mathrm{H}), 1.64-1.27(\mathrm{~m}, 7 \mathrm{H}), 1.27-1.12(\mathrm{~m}, 3 \mathrm{H}), 0.94-0.86(\mathrm{~m}$, 7H), 0.65 (s, 3H); ${ }^{13} \mathrm{C}$ NMR (75 MHz, $\mathrm{CDCl}_{3}$ ): $\delta$ 174.2, 126.5, 121.8, 82.1, 80.9, 77.1, 59.7, 56.0, 55.8, 55.5, 46.3, 46.2, 42.7, 42.1, 39.7, 35.4, 35.2, 35.0, 34.6, 31.1, 31.0, 28.1, 27.9, 27.5, 26.9, 23.3, 23.0, 22.1, 17.5, 12.6; IR (ATR): $v_{\max }$ 2934, 1739, 1372, 1159, 1101, 908, $732 \mathrm{~cm}^{-1}$; ESIHRMS $\mathrm{m} / z$ calcd for $\mathrm{C}_{30} \mathrm{H}_{50}{ }^{35} \mathrm{ClO}_{5}[\mathrm{M}+\mathrm{H}]^{+} 525.3341$, found 525.3336 .

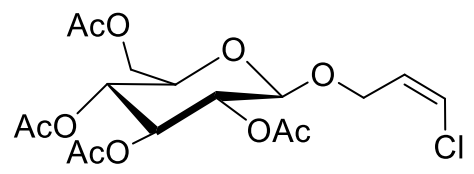

17

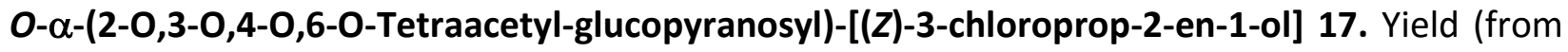
the corresponding iodoalkene - reaction performed on a $0.5 \mathrm{mmol}$ scale using $20 \mathrm{~mol} \%$ of copper iodide and 40 mol\% of trans- $N, N^{\prime}$-dimethylcyclohexane-1,2-diamine in dioxane for $48 \mathrm{~h}$ ): $60 \%$ (128 mg, $0.3 \mathrm{mmol}$ ). Solvent system for flash chromatography: petroleum ether/ethyl acetate: 70/30; White solid; $\mathrm{Mp}: 94{ }^{\circ} \mathrm{C} ;[\alpha]_{\mathrm{D}}^{25}+8.0\left(c\right.$ 1.0, $\left.\mathrm{CHCl}_{3}\right) ;{ }^{1} \mathrm{H} \mathrm{NMR}\left(\mathrm{CDCl}_{3}, 300 \mathrm{MHz}\right): \delta$ $6.17(\mathrm{~d}, J=7.2 \mathrm{~Hz}, 1 \mathrm{H}$ ), 5.87 (app. q, $J=6 \mathrm{~Hz}, 1 \mathrm{H}), 5.17$ (t, J=9.6 Hz, 1H), 5.05 (t, J = 9.6 Hz, 1H), $4.95(\mathrm{t}, J=8.1 \mathrm{~Hz}, 1 \mathrm{H}), 4.50(\mathrm{~d}, J=8.1 \mathrm{~Hz}, 1 \mathrm{H}), 4.40(\mathrm{td}, J=5.4 \mathrm{~Hz}$ and $1.2 \mathrm{~Hz}, 2 \mathrm{H}), 4.23(\mathrm{dd}, J=$ $12.3 \mathrm{~Hz}$ and $4.8 \mathrm{~Hz}, 1 \mathrm{H}), 4.10(\mathrm{dd}, J=12.3 \mathrm{~Hz}$ and $2.1 \mathrm{~Hz}, 1 \mathrm{H}), 3.67(\mathrm{dq}, J=9.9 \mathrm{~Hz}$ and $2.4 \mathrm{~Hz}, 1 \mathrm{H}$ ),

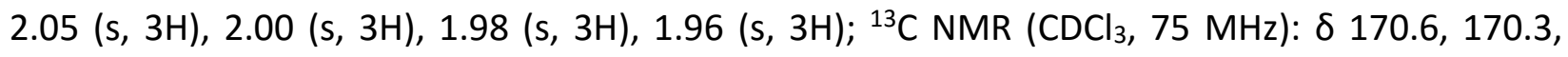
169.3 (2C), 127.6, 121.4, 99.9, 72.9, 72.0, 71.3, 68.4, 64.6, 61.9, 20.8 (2C), 20.6 (2C); IR (ATR): $V_{\max }\left(\mathrm{cm}^{-1}\right) 1747,1364,1219,1163,1036,906,758 \mathrm{~cm}^{-1}$; ESIHRMS m/z calcd for $\mathrm{C}_{17} \mathrm{H}_{24}{ }^{35} \mathrm{ClO}_{10}$ $[\mathrm{M}+\mathrm{H}]^{+}$423.1053, found 423.1048 . 


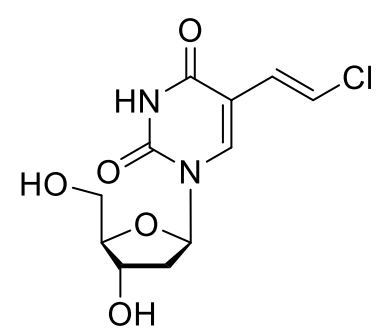

19

(E)-5-(2-Chlorovinyl)-2'-deoxyuridine 19. Yield (from the corresponding bromoalkene - reaction performed on a $0.5 \mathrm{mmol}$ scale): $60 \%$ (87 $\mathrm{mg}, 0.30 \mathrm{mmol})$. Solvent system for flash chromatography: EtOAc. This compound has been previously reported. ${ }^{58}$

s8 M. Johar, T. Mannin, D. Y. Kunimoto, R. Kumar, Bioorg. Med. Chem. 2005, 13, 6663. 


\section{Experimental Procedures and Characterization Data: Copper-Catalyzed I $\rightarrow$ Br Exchange from Alkenyl lodides}

\section{General procedure}

An oven dried $15 \mathrm{~mL}$ resealable pressure tube was charged with copper iodide (19 $\mathrm{mg}, 0.1$ $\mathrm{mmol})$, tetramethylammonium bromide $(616 \mathrm{mg}, 4.0 \mathrm{mmol})$ and the iodoalkene $(1.0 \mathrm{mmol})$. The tube was fitted with a rubber septum, evacuated under vacuum, backfilled with argon and trans- $N, N^{\prime}$-dimethylcyclohexane-1,2-diamine (31 $\mu \mathrm{L}, 0.2 \mathrm{mmol}$ ) and ethanol (or DMSO when specified) $(2 \mathrm{~mL})$ were next added. The resealable pressure tube was closed with a Tefloncoated screw cap and the resulting suspension was stirred and heated at $110^{\circ} \mathrm{C}$ in a preheated oil bath for $72 \mathrm{~h}$ (unless specified otherwise). When the reaction was run in ethanol, the crude reaction mixture was cooled to $\mathrm{rt}$, diluted with ethyl acetate, filtered on a plug of silica gel and concentrated. When the reaction was run in DMSO, the crude reaction mixture was cooled to rt, diluted with water $(15 \mathrm{~mL})$, extracted thrice with diethyl ether and the combined organic layers were dried over anhydrous magnesium sulfate, filtered and concentrated. The crude residue was finally purified by flash column chromatography over silica gel to afford the desired bromoalkene.

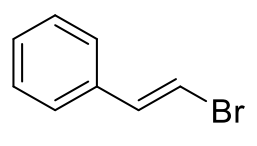

$11 \mathrm{a}$

(E)-(2-Bromovinyl)benzene 11a. Yield: $85 \%$ (154 mg, $0.85 \mathrm{mmol}$ ). Solvent system for flash chromatography: pentane. Colorless oil. This compound has been previously reported. ${ }^{\mathrm{S} 4}$ 


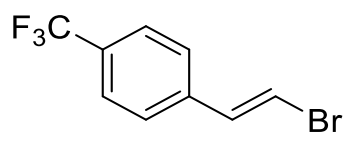

11b

(E)-1-(2-Bromovinyl)-4-(trifluoromethyl)benzene 11b. Yield: $80 \%$ (200 mg, $0.80 \mathrm{mmol})$. Solvent system for flash chromatography: pentane. This compound has been previously reported. ${ }^{\mathrm{S9}}$

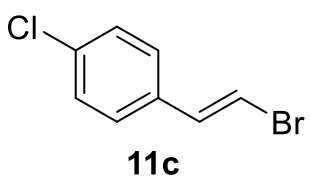

(E)-1-(2-Bromovinyl)-4-chlorobenzene 11c. Yield: 75\% (163 mg, $0.75 \mathrm{mmol}$ ). Solvent system for flash chromatography: pentane. This compound has been previously reported. ${ }^{59}$

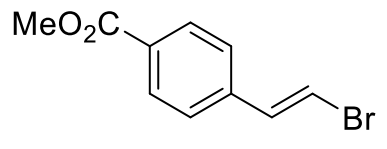

$11 \mathrm{~d}$

(E)-Methyl-4-(2-bromovinyl)benzoate 11d. Yield (reaction performed on a $0.5 \mathrm{mmol}$ scale): $76 \%$

(92 mg, $0.38 \mathrm{mmol}$ ). Solvent system for flash chromatography: petroleum ether/EtOAc: 98/2.

This compound has been previously reported. ${ }^{59}$

s9 D. Chang, Y. Gu, Q. Shen, Chem. Eur. J. 2015, 21, 6074. 


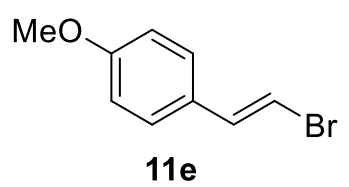

(E)-(2-Bromovinyl)-4-methoxybenzene 11e. Yield: $58 \%$ (122 mg, $0.58 \mathrm{mmol})$. Solvent system for flash chromatography: cyclohexane/EtOAc: 95/5. This compound has been previously reported. ${ }^{5} 4$

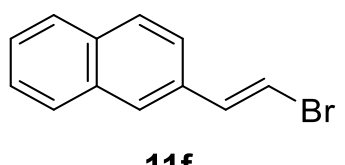

$11 f$

(E)-2-(2-Bromovinyl)naphthalene 11f. Yield (reaction performed on a $0.5 \mathrm{mmol}$ scale): $75 \%$ (87 $\mathrm{mg}, 0.38 \mathrm{mmol}$ ). Solvent system for flash chromatography: pentane. This compound has been previously reported. ${ }^{\mathrm{S} 10}$

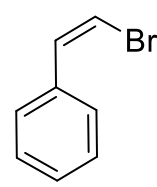

$11 \mathrm{~g}$

(Z)-(2-Bromovinyl)benzene 11g. Yield: 72\% (131 mg, $0.72 \mathrm{mmol})$. Solvent system for flash chromatography: pentane. This compound has been previously reported. ${ }^{\text {S11 }}$

${ }^{10}$ Y. R. Bhorge, S.-H. Chang, C.-T. Chang, T.-H. Yan, Tetrahedron 2012, 68, 4846.

${ }^{11}$ J. Uenishi, R. Kawahama, O. Yonemitsu, J. Org. Chem. 1998, 63, 8965. 


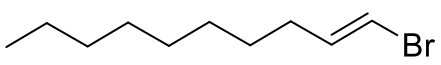

$11 \mathrm{i}$

(E)-1-Bromodec-1-ene 11i. Yield: $64 \%(140 \mathrm{mg}, 0.64 \mathrm{mmol})$. Solvent system for flash chromatography: pentane; Colorless oil; ${ }^{1} \mathrm{H}$ NMR $\left(300 \mathrm{MHz}, \mathrm{CDCl}_{3}\right): \delta 6.17$ (dt, $J=13.5 \mathrm{~Hz}$ and $7.2 \mathrm{~Hz}, 1 \mathrm{H}$ ), 6.00 (dt, $J=13.5 \mathrm{~Hz}$ and $1.2 \mathrm{~Hz}, 1 \mathrm{H}$ ), 2.03 (app. qd, $J=7.2 \mathrm{~Hz}$ and $0.9 \mathrm{~Hz}, 2 \mathrm{H}$ ), 1.44$1.37(\mathrm{~m}, 2 \mathrm{H}), 1.32-1.21(\mathrm{~m}, 10 \mathrm{H}), 0.88(\mathrm{t}, J=6.3 \mathrm{~Hz}, 3 \mathrm{H}) ;{ }^{13} \mathrm{C} \mathrm{NMR}\left(75 \mathrm{MHz}, \mathrm{CDCl}_{3}\right): \delta 138.5$, 104.1, 33.1, 32.0, 29.5, 29.4, 29.1, 28.8, 22.8, 14.2; IR (ATR): $v_{\max } 2955,2925,2854,1620,1377$, $1216,933,735,705 \mathrm{~cm}^{-1}$; EIHRMS $\mathrm{m} / z$ calcd for $\mathrm{C}_{10} \mathrm{H}_{19}{ }^{79} \mathrm{Br}[\mathrm{M}]^{+} 218.0665$, found 218.0667 .

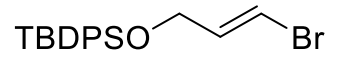

$11 \mathrm{j}$

(E)-3-Bromo-1-tert-butyldiphenylsilyloxy-prop-2-ene 11j. Yield (reaction performed on a 0.2 mmol scale): $85 \%$ (64 $\mathrm{mg}, 0.17 \mathrm{mmol})$. Solvent system for flash chromatography: cyclohexane/ethyl acetate: 98/2; Colorless oil. This compound has been previously reported. ${ }^{\mathrm{S12}}$

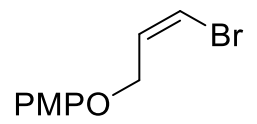

111

(Z)-3-Bromo-1-(4-methoxy)phenyloxy-prop-2-ene 11l. Yield (reaction performed on a $0.5 \mathrm{mmol}$ scale using 20 mol\% of copper iodide and 40 mol\% of trans- $N, N^{\prime}$-dimethylcyclohexane-1,2diamine in DMSO for $48 \mathrm{~h}$ ): 54\% (66 mg, $0.27 \mathrm{mmol}$ ). Solvent system for flash chromatography: pentane/Et ${ }_{2} \mathrm{O}$ : 98/2; Brown oil; ${ }^{1} \mathrm{H}$ NMR (300 MHz, $\left.\mathrm{CDCl}_{3}\right): \delta 6.84(\mathrm{~s}, 4 \mathrm{H}), 6.47-6.33$ (m, $\left.2 \mathrm{H}\right), 4.66$ (dd, $J=5.1 \mathrm{~Hz}$ and $1.1 \mathrm{~Hz}, 2 \mathrm{H}), 3.77(\mathrm{~s}, 3 \mathrm{H}) ;{ }^{13} \mathrm{C} \mathrm{NMR}\left(75 \mathrm{MHz}, \mathrm{CDCl}_{3}\right): \delta 154.3,152.4,131.6$,

\footnotetext{
s12 R. E. Maleczka, W. P. Gallagher, Org. Lett. 2001, 3, 4173.
} 
115.8, 114.8, 109.7, 67.0, 55.9; IR (ATR): $V_{\max } 2832,1505,1461,1290,1227,1180,1038,823$, 753, $676 \mathrm{~cm}^{-1}$; EIHRMS $\mathrm{m} / \mathrm{z}$ calcd for $\mathrm{C}_{10} \mathrm{H}_{11}{ }^{79} \mathrm{BrO}_{2}$ [M] ${ }^{+}$241.9937, found 241.9947.

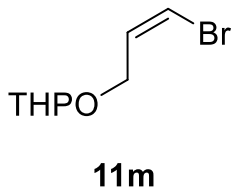

(Z)-3-Bromo-1-(2-Tetrahydro-2H-pyranyloxy)-prop-2-ene 11m. Yield: 68\% (151 mg, $0.68 \mathrm{mmol})$. Solvent system for flash chromatography: pentane/Et $2 \mathrm{O}: 80 / 20$; Brown oil; ; ${ }^{1} \mathrm{H}$ NMR (300 MHz, $\left.\mathrm{CDCl}_{3}\right): \delta 6.32(\mathrm{~m}, 2 \mathrm{H}), 4.65(\mathrm{t}, J=3.9 \mathrm{~Hz}, 1 \mathrm{H}), 4.36$ (A of ABXY syst., $J=6.7 \mathrm{~Hz}$ and $4.9 \mathrm{~Hz}$ and 1.3 $\mathrm{Hz}, 1 \mathrm{H}), 4.21$ (B of ABXY syst., $J=7.3 \mathrm{~Hz}$ and $5.7 \mathrm{~Hz}$ and $1.2 \mathrm{~Hz}, 1 \mathrm{H}), 3.88(\mathrm{~m}, 1 \mathrm{H}), 3.53(\mathrm{~m}, 1 \mathrm{H})$, 1.91-1.43 (m, 6H); ${ }^{13} \mathrm{C} \mathrm{NMR}\left(75 \mathrm{MHz}, \mathrm{CDCl}_{3}\right): \delta 129.0,120.1,98.7,62.9,62.5,30.7,25.5,19.6 ; \mathrm{IR}$ (ATR): $v_{\max } 2942,1634,1454,1351,1201,1156,1120,1070,1029,964,906,870,815,733,683$ $\mathrm{cm}^{-1}$; ElHRMS $\mathrm{m} / \mathrm{z}$ calcd for $\mathrm{C}_{8} \mathrm{H}_{13}{ }^{79} \mathrm{BrO}_{2}[\mathrm{M}]^{+} 220.0093$, found 220.0096 .

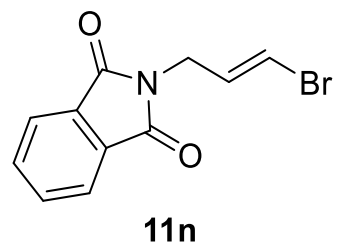

(E)-3-Bromo-1-phthalimido-prop-2-ene 11n. Yield (reaction performed on a $0.5 \mathrm{mmol}$ scale): $85 \%$ (113 mg, $0.42 \mathrm{mmol}$ ). Solvent system for flash chromatography: petroleum ether/ethyl acetate: 80/20; White solid; $\mathrm{Mp}: 109^{\circ} \mathrm{C} ;{ }^{1} \mathrm{H}$ NMR $\left(300 \mathrm{MHz}, \mathrm{CDCl}_{3}\right.$ ): $\delta 7.85$ (dd, $J=5.4 \mathrm{~Hz}$ and 3 $\mathrm{Hz}, 2 \mathrm{H}), 7.71(\mathrm{dd}, J=5.4 \mathrm{~Hz}$ and $3 \mathrm{~Hz}, 2 \mathrm{H}), 6.45(\mathrm{dt}, J=13.5 \mathrm{~Hz}$ and $0.9 \mathrm{~Hz}, 1 \mathrm{H}), 6.27$ (app. dt, $J=$ 13.8 $\mathrm{Hz}$ and $6.6 \mathrm{~Hz}, 1 \mathrm{H}), 4.24(\mathrm{dd}, J=6.6 \mathrm{~Hz}$ and $0.9 \mathrm{~Hz}, 2 \mathrm{H}) ;{ }^{13} \mathrm{C} \mathrm{NMR}\left(75 \mathrm{MHz}, \mathrm{CDCl}_{3}\right): \delta 167.7$, 134.3, 132.1, 130.9, 123.6, 111.0, 39.1; IR (ATR): $V_{\max } 2926,1705,1466,1430,1392,1344,1307$, 1222, 1039, 941, 928, $712 \mathrm{~cm}^{-1}$; ESIHRMS m/z calcd for $\mathrm{C}_{11} \mathrm{H}_{9}{ }^{79} \mathrm{BrNO}_{2}[\mathrm{M}+\mathrm{H}]^{+} 265.9811$, found 265.9806 . 


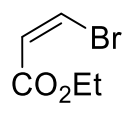

110

(Z)-Ethyl-4-bromobut-3-enoate 110. This compound is extremely volatile and was isolated together with $13 \%$ of the inseparable starting iodide. NMR Yield using DMF as an internal standard: $55 \%$. Solvent system for flash chromatography: pentane. This compound has been previously reported. ${ }^{\mathrm{S7}}$

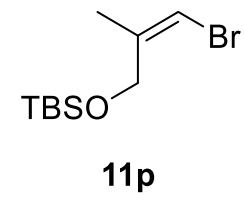

(Z)-3-Bromo-1-tert-butyldimethylsilyloxy-2-methyl-prop-2-ene 11p. Yield: 83\% (209 mg, 0.83 mmol). Solvent system for flash chromatography: pentane/Et $2 \mathrm{O}: 98 / 2$; Colorless oil; ${ }^{1} \mathrm{H} N M R$ $\left(300 \mathrm{MHz}, \mathrm{CDCl}_{3}\right): \delta 5.86(\mathrm{t}, J=1.2 \mathrm{~Hz}, 1 \mathrm{H}), 4.30(\mathrm{~s}, 2 \mathrm{H}), 1.82(\mathrm{~s}, 3 \mathrm{H}), 0.91(\mathrm{~s}, 9 \mathrm{H}), 0.09(\mathrm{~s}, 6 \mathrm{H}) ;{ }^{13} \mathrm{C}$ NMR (75 MHz, $\mathrm{CDCl}_{3}$ ): $\delta 141.4,99.9,64.2,26.0,19.9,18.4,-5.2 ;$ IR (ATR): $v_{\max } 2954,2929,2856$, 1471, 1252, 1090, 836, 776, 710, $667 \mathrm{~cm}^{-1}$; ElHRMS m/z calcd for $\mathrm{C}_{10} \mathrm{H}_{21}{ }^{79} \mathrm{BrOSi}[\mathrm{M}]^{+}$264.0540, found 264.0544 .

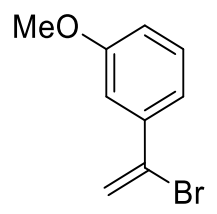

$11 q$

1-(1-Bromovinyl)-3-methoxybenzene 11q. Yield (reaction performed on a $0.5 \mathrm{mmol}$ scale using $20 \mathrm{~mol} \%$ of copper iodide and $40 \mathrm{~mol} \%$ of trans- $N, N^{\prime}$-dimethylcyclohexane-1,2-diamine in 
DMSO for $48 \mathrm{~h}): 75 \%$ (80 $\mathrm{mg}, 0.38 \mathrm{mmol})$. Solvent system for flash chromatography: pentane. This compound has been previously reported. ${ }^{\mathrm{S} 13}$

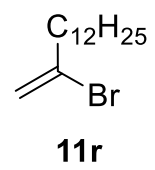

2-Bromotetradec-1-ene 11r. Yield (reaction performed on a $0.5 \mathrm{mmol}$ scale using $20 \mathrm{~mol} \%$ of copper iodide and 40 mol\% of trans- $N, N^{\prime}$-dimethylcyclohexane-1,2-diamine in DMSO for $48 \mathrm{~h}$ ): $88 \%$ (122 mg, $0.44 \mathrm{mmol}$ ). Solvent system for flash chromatography: pentane; Colorless oil. This compound has been previously reported. ${ }^{\text {S14 }}$

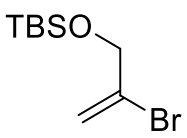

$11 \mathrm{~s}$

2-Bromo-1-tert-butyldimethylsilyloxy-prop-2-ene 11s. Yield (reaction performed on a $0.5 \mathrm{mmol}$ scale using 20 mol\% of copper iodide and 40 mol\% of trans- $N, N^{\prime}$-dimethylcyclohexane-1,2diamine in DMSO for $48 \mathrm{~h}$ ): $64 \%$ (80 $\mathrm{mg}, 0.32 \mathrm{mmol}$ ): $64 \%$ (80 $\mathrm{mg}, 0.32 \mathrm{mmol}$ ). Solvent system for flash chromatography: pentane/Et $2 \mathrm{O}: 98 / 2$. This compound has been previously reported. ${ }^{\mathrm{S} 15}$

s13 F. Gao, A. H. Hoveyda, J. Am. Chem. Soc. 2010, 132, 10961.

S14 K. Blatte, A.-D. Schlüter, Synthesis 1989, 356.

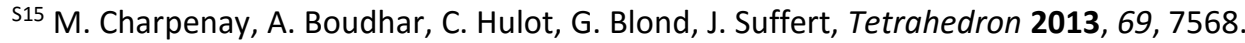




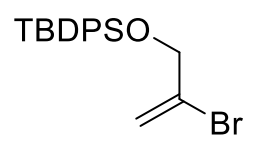

$11 t$

2-Bromo-1-tert-butyldiphenylsilyloxy-prop-2-ene 11t. Yield (reaction performed on a $0.5 \mathrm{mmol}$ scale using 20 mol\% of copper iodide and 40 mol\% of trans- $N, N^{\prime}$-dimethylcyclohexane-1,2diamine in DMSO for 48h): $87 \%$ (122 mg, $0.44 \mathrm{mmol})$. Solvent system for flash chromatography: pentane. This compound has been previously reported. ${ }^{\mathrm{S} 16}$

${ }^{\text {s16 }}$ B. Trost, M. R. Machacek, H. C. Tsui, J. Am. Chem. Soc. 2005, 127, 7014. 


\section{Experimental Procedures and Characterization Data: Copper-Catalyzed $\mathrm{Br} \rightarrow \mathrm{Cl}$ Exchange from gem-Dibromoalkenes}

\section{General procedure}

An oven dried $15 \mathrm{~mL}$ resealable pressure tube was charged with copper iodide (28 $\mathrm{mg}, 0.15$ $\mathrm{mmol})$, tetramethylammonium chloride $(274 \mathrm{mg}, 2.5 \mathrm{mmol})$ and the gem-dibromoalkene $(0.5$ mmol). The tube was fitted with a rubber septum, evacuated under vacuum, backfilled with argon and trans- $N, N^{\prime}$-dimethylcyclohexane-1,2-diamine (47 $\left.\mu \mathrm{L}, 0.3 \mathrm{mmol}\right)$ and DMSO (1 mL) were next added. The resealable pressure tube was closed with a Teflon-coated screw cap and the resulting suspension was stirred and heated at $110^{\circ} \mathrm{C}$ in a preheated oil bath for $96 \mathrm{~h}$. The resulting mixture was cooled, diluted with water $(15 \mathrm{~mL})$, extracted thrice with diethyl ether and the combined organic layers were dried over anhydrous magnesium sulfate, filtered and concentrated. The crude residue was finally purified by flash column chromatography over silica gel to afford the desired vinyl dichloride.

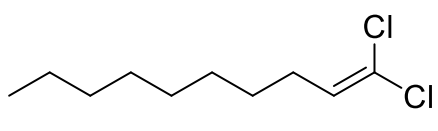

$13 a$

1,1-Dichlorodec-1-ene 13a. Yield: $44 \%$ (46 mg, $0.22 \mathrm{mmol}$ ). Solvent system for flash chromatography: pentane; Colorless oil. This compound has been previously reported. ${ }^{\text {S17 }}$

S17 Z. Wang, J. M. Fortunal, Process for the preparation of cyclopropylacetylene, US Patent 6,359,164. March 19, 2002. 


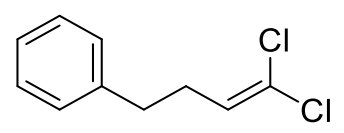

$13 b$

(4,4-Dichlorobut-3-en-1-yl)benzene 13b. Yield: 64\% (64 mg, $0.32 \mathrm{mmol}$ ). Contaminated with 10 $\%$ of the corresponding bromo-chloro-alkene. Solvent system for flash chromatography: pentane; Colorless oil; ${ }^{1} \mathrm{H}$ NMR $\left(300 \mathrm{MHz}, \mathrm{CDCl}_{3}\right): \delta$ 7.35-7.27 (m, 2H), 7.25-7.16 (m, 3H), $5.88(\mathrm{t}, J=$ $7.2 \mathrm{~Hz}, 1 \mathrm{H}$ ), $2.73(\mathrm{t}, J=7.5 \mathrm{~Hz}, 2 \mathrm{H}), 2.50$ (app. q, $J=7.5 \mathrm{~Hz}, 2 \mathrm{H}) ;{ }^{13} \mathrm{C} \mathrm{NMR}\left(75 \mathrm{MHz}, \mathrm{CDCl}_{3}\right): \delta$ $140.8,129.0,128.7,128.5,126.4,120.8,34.4,31.4$; IR (ATR): $v_{\max } 3028,1691,1496,1454,1084$, 1030, 878, 746, $698 \mathrm{~cm}^{-1}$; ElHRMS $\mathrm{m} / z$ calcd for $\mathrm{C}_{10} \mathrm{H}_{10}{ }^{35} \mathrm{Cl}_{2}[\mathrm{M}]^{+} 200.0154$, found 200.0153.

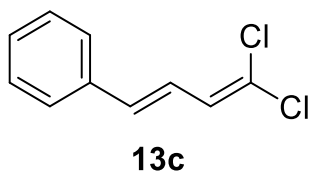

(E)-1-(4,4-Dichlorobuta-1,3-dienyl)benzene 13c. Yield (using 20 mol\% of copper iodide and 40 mol\% of trans- $N, N^{\prime}$-dimethylcyclohexane-1,2-diamine in DMSO for $48 \mathrm{~h}$ ): $74 \%$ (74 mg, 0.37 mmol). Solvent system for flash chromatography: pentane; Pale yellow oil; ${ }^{1} \mathrm{H}$ NMR (300 MHz, $\left.\mathrm{CDCl}_{3}\right): \delta 7.45(\mathrm{~d}, J=7.5 \mathrm{~Hz}, 2 \mathrm{H}), 7.39-7.27(\mathrm{~m}, 3 \mathrm{H}), 6.89(\mathrm{dd}, J=15.6 \mathrm{~Hz}$ and $10.5 \mathrm{~Hz}, 1 \mathrm{H}), 6.65$ $(\mathrm{d}, J=15.9 \mathrm{~Hz}, 1 \mathrm{H}), 6.58(\mathrm{~d}, J=10.5 \mathrm{~Hz}, 1 \mathrm{H}) ;{ }^{13} \mathrm{C} \mathrm{NMR}\left(75 \mathrm{MHz}, \mathrm{CDCl}_{3}\right): \delta$ 136.5, 135.3, 129.3, 128.9, 128.6, 126.9, 122.9, 121.6; IR (ATR): $v_{\max }$ 3037, 1701, 1494, 1453, 1158, 966, 903, 749, $696 \mathrm{~cm}^{-1}$; EIHRMS $\mathrm{m} / \mathrm{z}$ calcd for $\mathrm{C}_{10} \mathrm{H}_{8}{ }^{35} \mathrm{Cl}_{2}[\mathrm{M}]^{+}$197.9998, found 198.0000 .

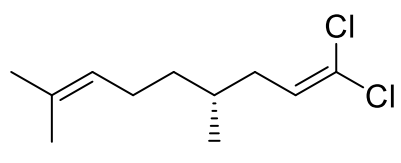

$13 d$

(R)-1,1-Dichloro-4,8-dimethylnona-1,7-diene 13d. Yield: 57\% (64 mg, $0.28 \mathrm{mmol}$ ). Solvent system for flash chromatography: pentane; Pale yellow oil; $[\alpha]_{\mathrm{D}}^{25}-3.5$ (c 1.0, $\mathrm{CHCl}_{3}$ ); ${ }^{1} \mathrm{H} \mathrm{NMR}$ 
(300 MHz, $\left.\mathrm{CDCl}_{3}\right): \delta 5.86(\mathrm{t}, J=7.5 \mathrm{~Hz}, 1 \mathrm{H}), 5.08(\mathrm{t}, J=6.6 \mathrm{~Hz}, 1 \mathrm{H}), 2.24-2.11(\mathrm{~m}, 1 \mathrm{H}), 2.09-1.92$ (m, 3H), $1.68(\mathrm{~s}, 3 \mathrm{H}), 1.60$ (br. s, 4H), 1.40-1.28 (m, 1H), 1.26-1.12 (m, 1H), $0.91(\mathrm{~d}, J=6.6 \mathrm{~Hz}$, 3H); ${ }^{13} \mathrm{C} \mathrm{NMR}\left(75 \mathrm{MHz}, \mathrm{CDCl}_{3}\right): \delta 131.6,129.0,124.6,120.3,36.9,36.8,32.5,25.9,25.7,19.6$, 17.8; IR (ATR): $v_{\max } 2963,2914,1619,1453,1378,1118,914,853,621 \mathrm{~cm}^{-1}$; ElHRMS m/z calcd for $\mathrm{C}_{11} \mathrm{H}_{18}{ }^{35} \mathrm{Cl}_{2}[\mathrm{M}]^{+}$220.0780, found 220.0776 . 


\section{${ }^{1} \mathrm{H}$ and ${ }^{13} \mathrm{C}$ NMR Spectra}




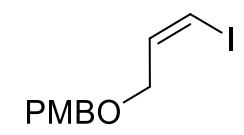

9|
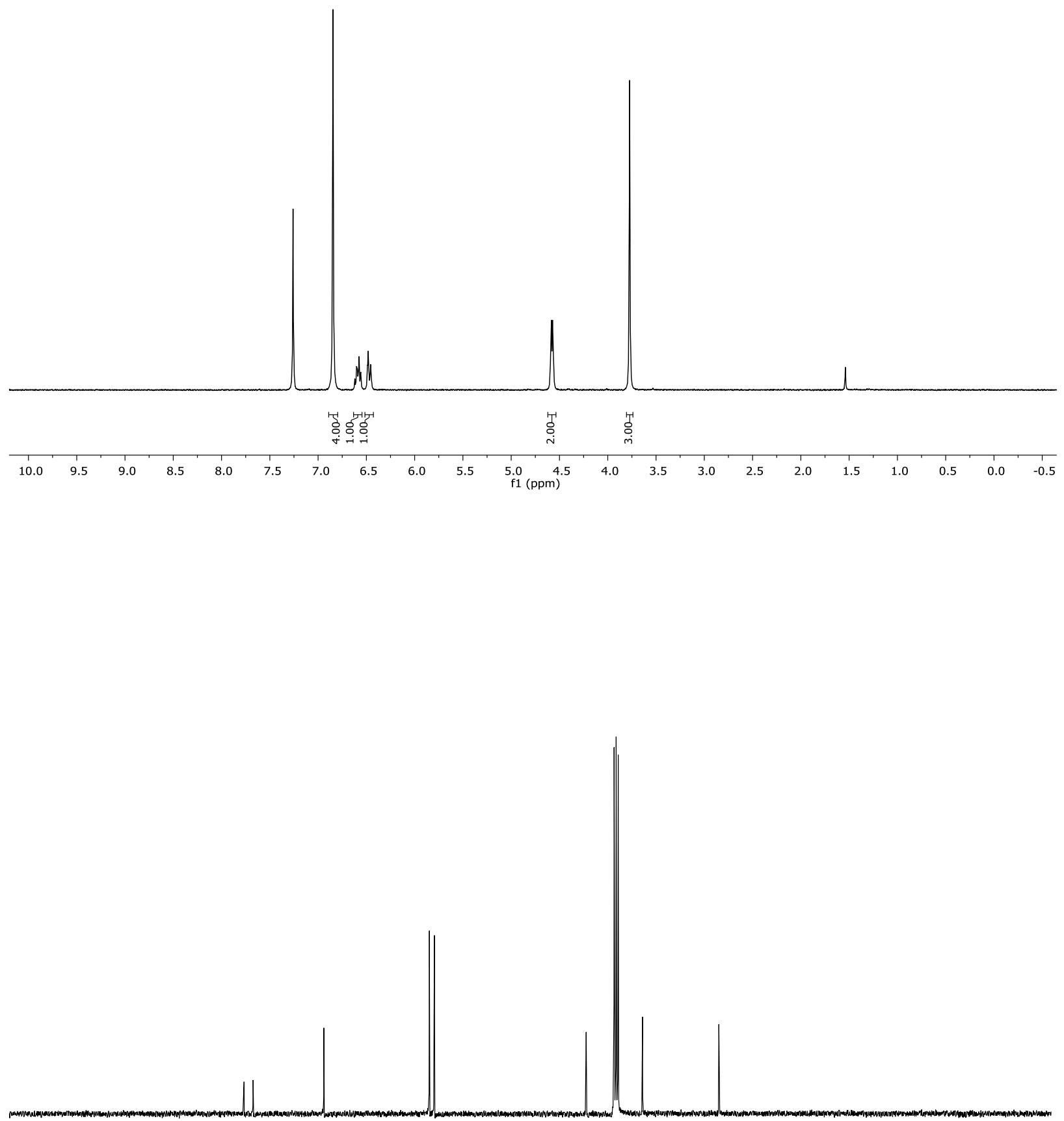

$\begin{array}{llllllllll}190 & 180 & 170 & 160 & 150 & 140 & 130 & 120 & 110 & 100 \\ \mathrm{f} 1(\mathrm{ppm})\end{array}$ 80 70 60 50 40 $\begin{array}{lllll}30 & 20 & 10 & 0 & -10\end{array}$ 
TBDPSO<smiles>C=C(I)CCC</smiles>

9t
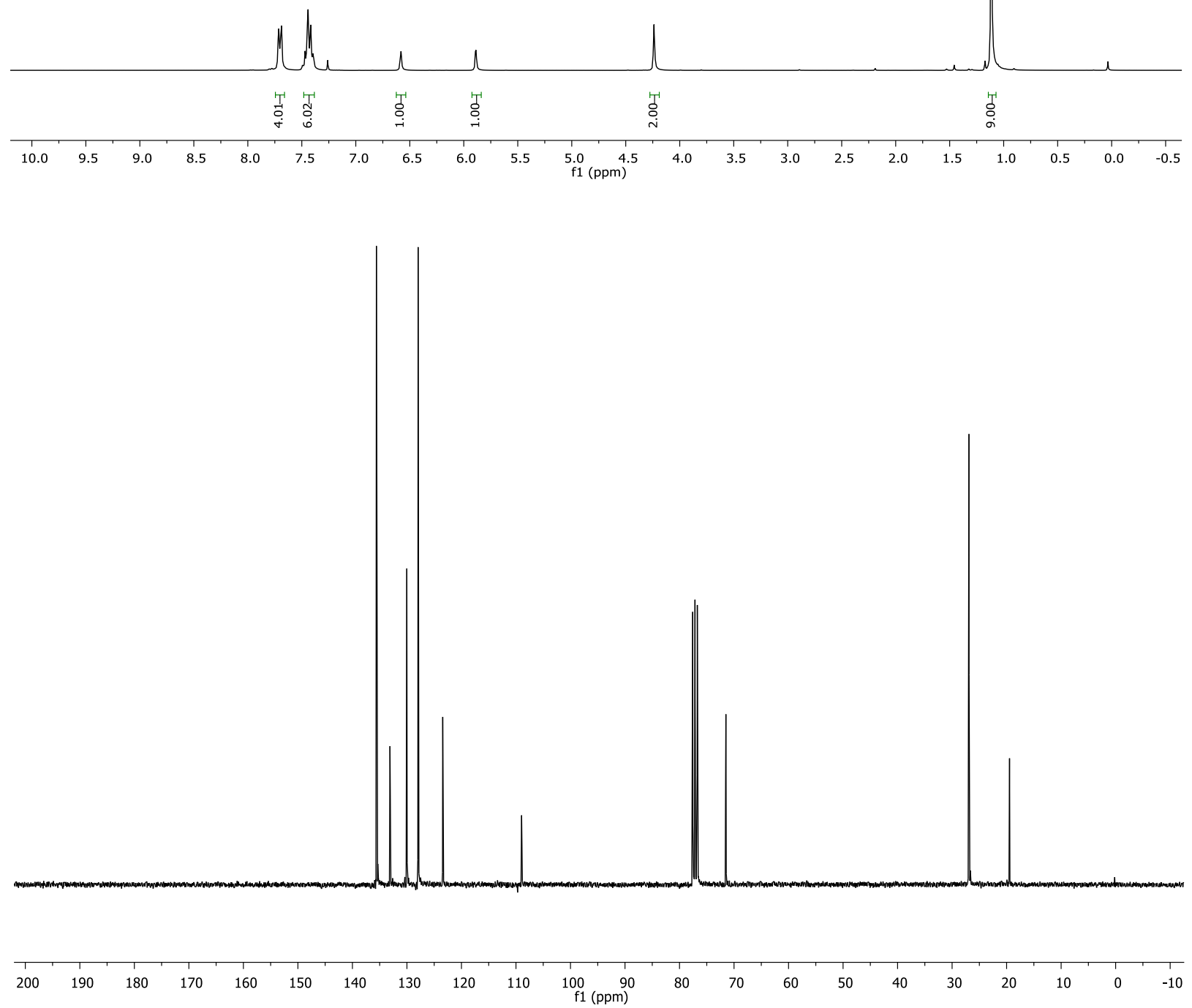


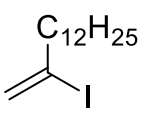

$9 r$
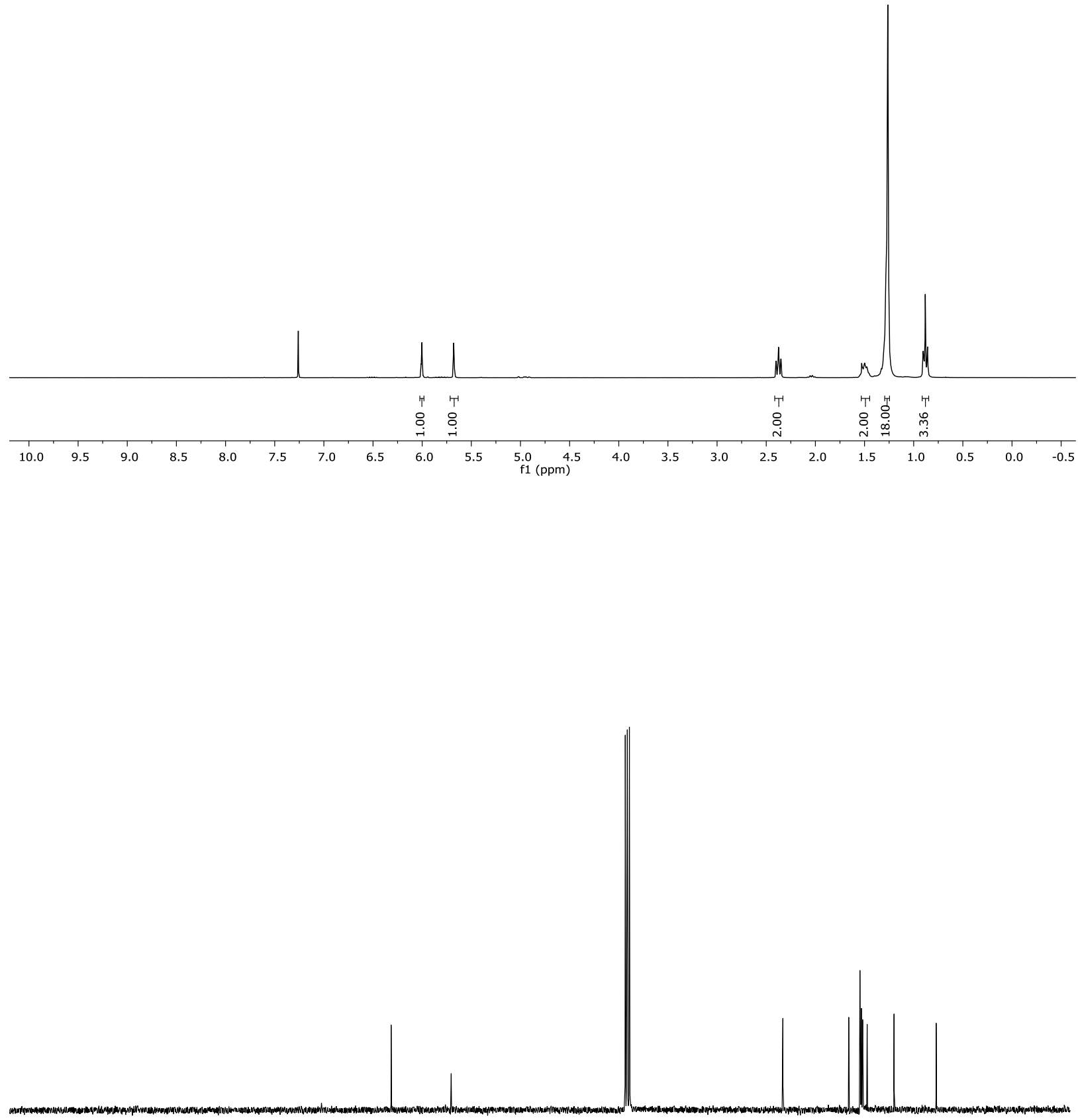

$\begin{array}{lllllllllll}200 & 190 & 180 & 170 & 160 & 150 & 140 & 130 & 120 & 110 & \begin{array}{c}100 \\ \text { f1 (ppm) }\end{array}\end{array}$ 

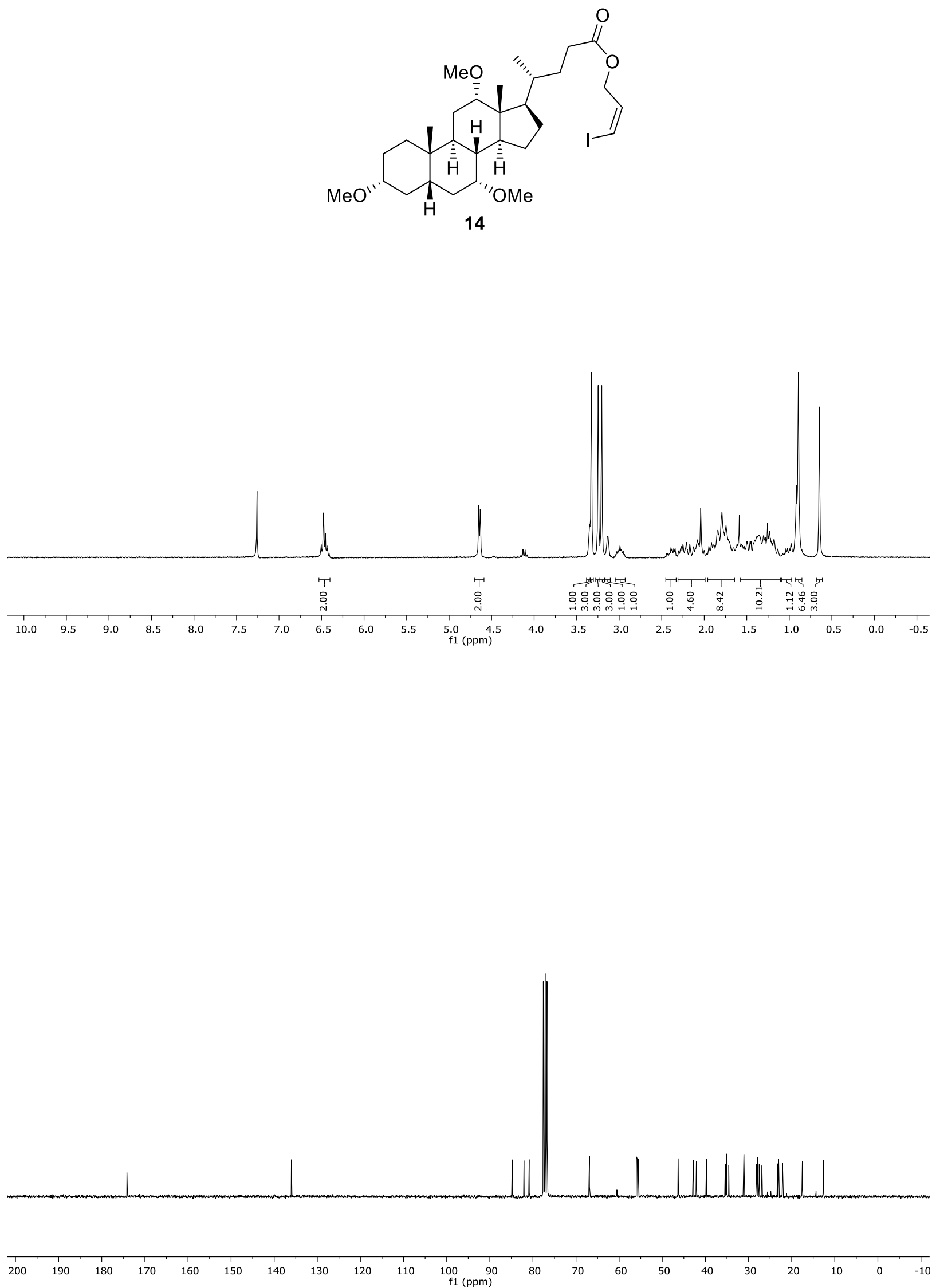


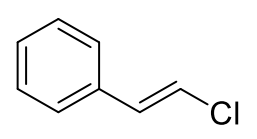

$10 a$
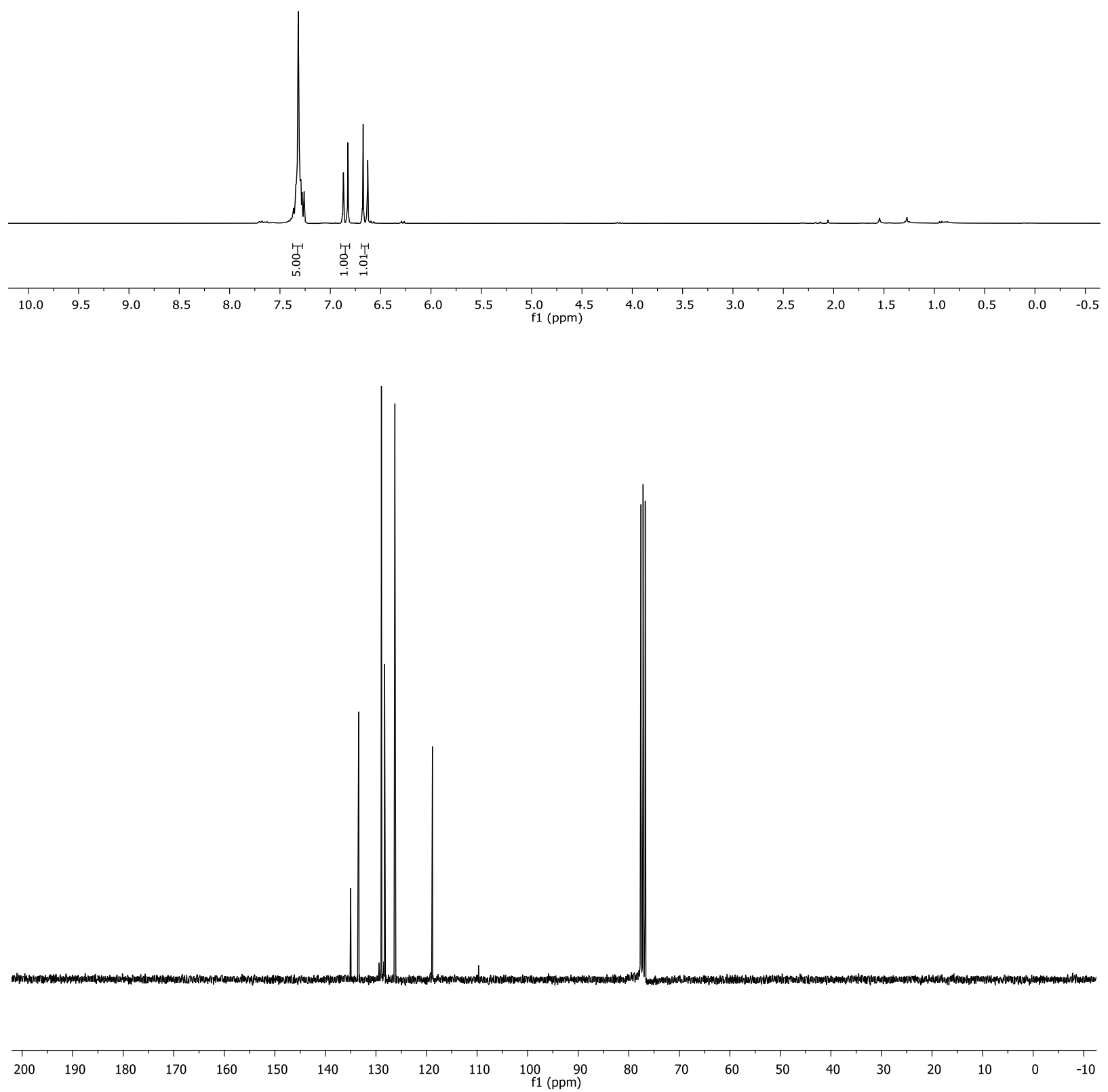


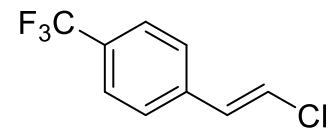

$10 \mathrm{~b}$
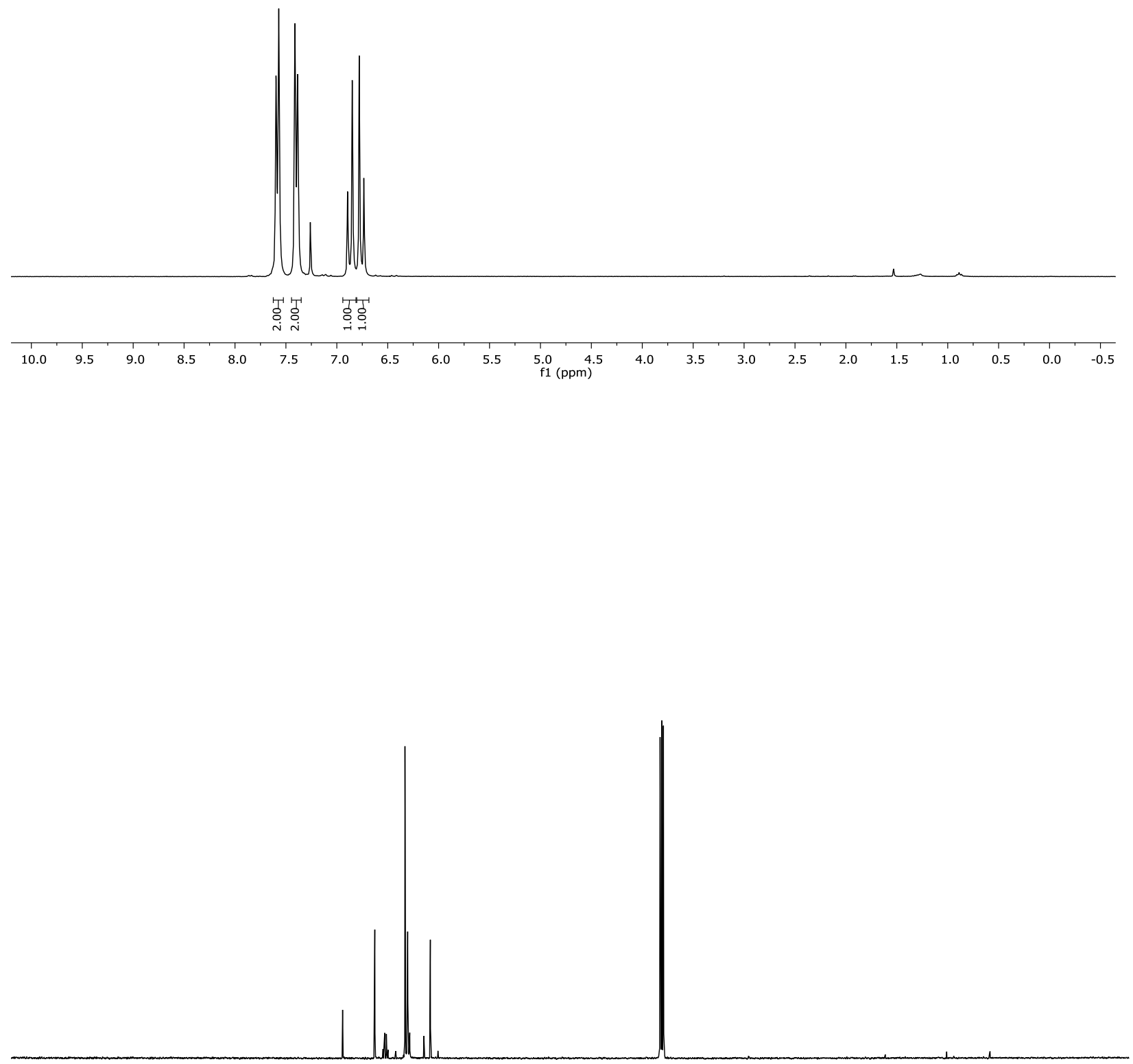$$
\text { T }
$$ 


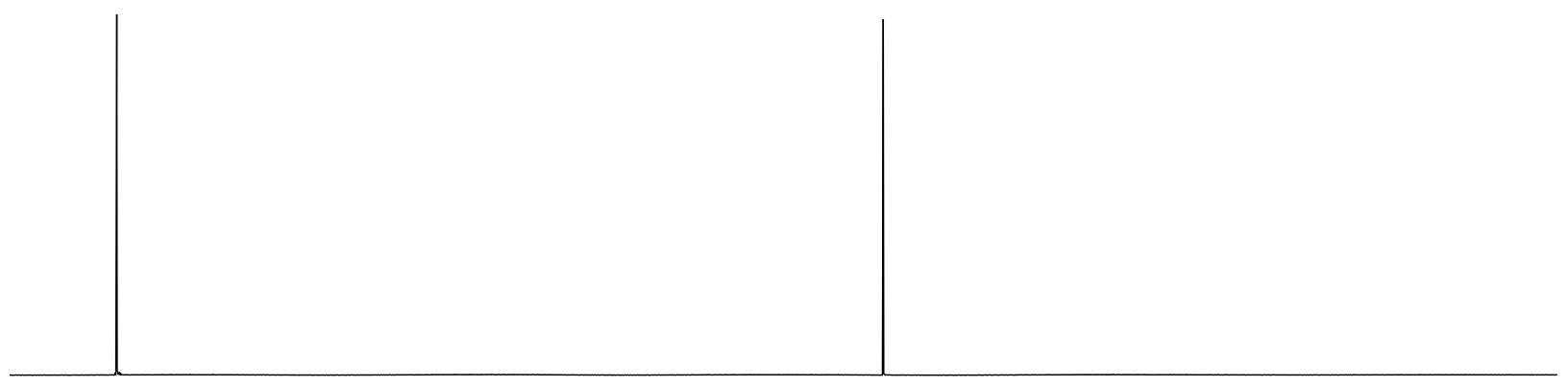

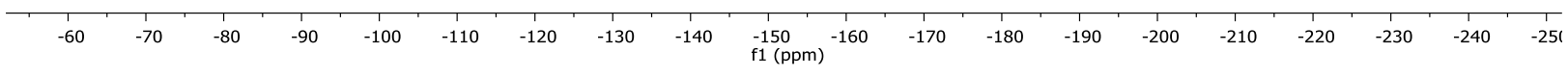




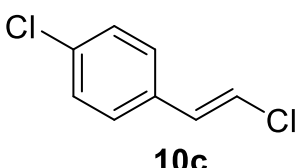

$10 \mathrm{c}$
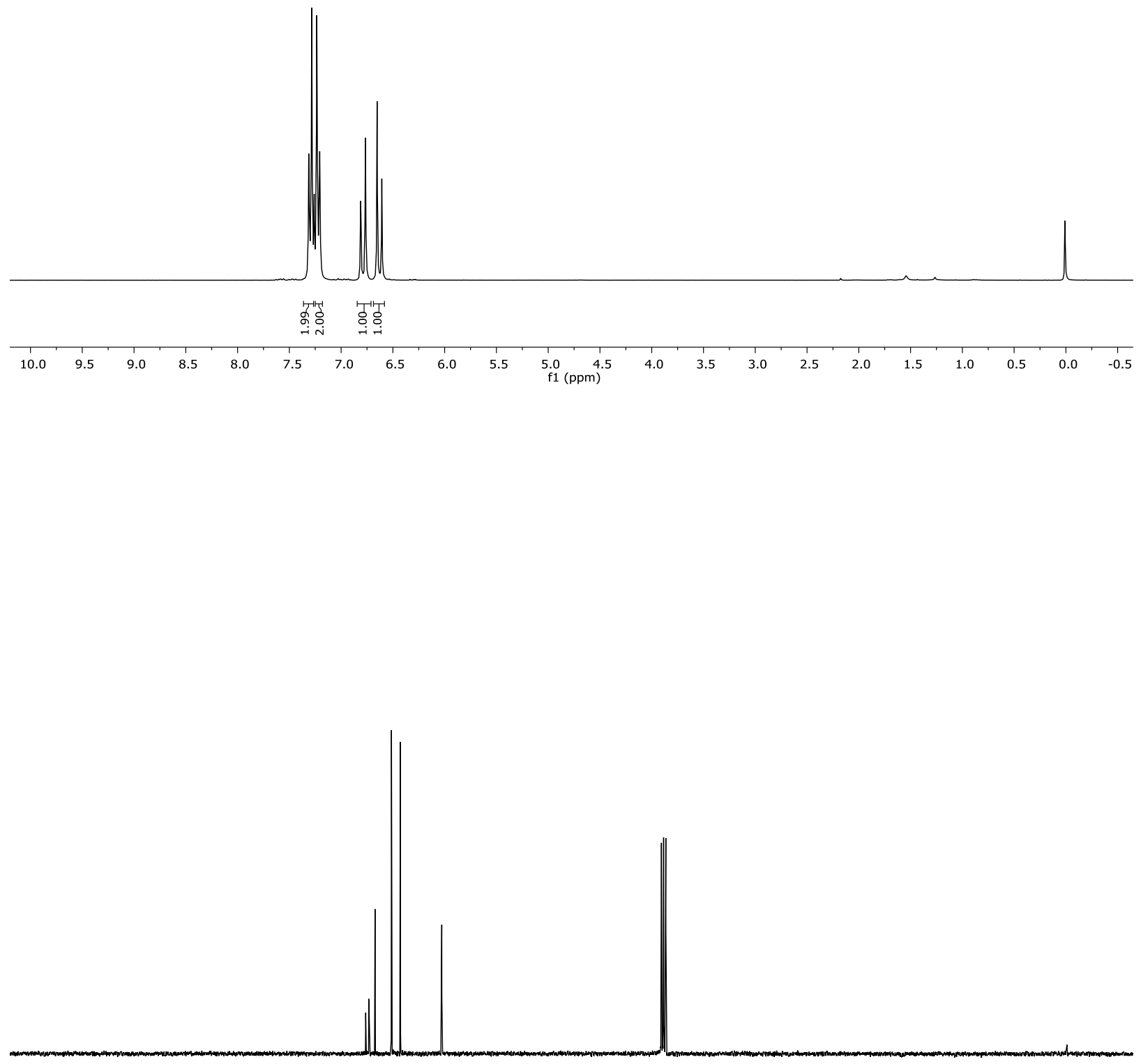

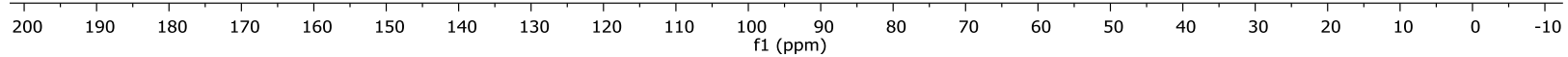




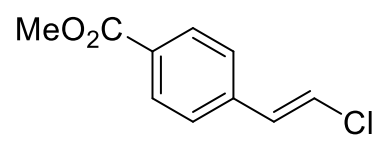

10d
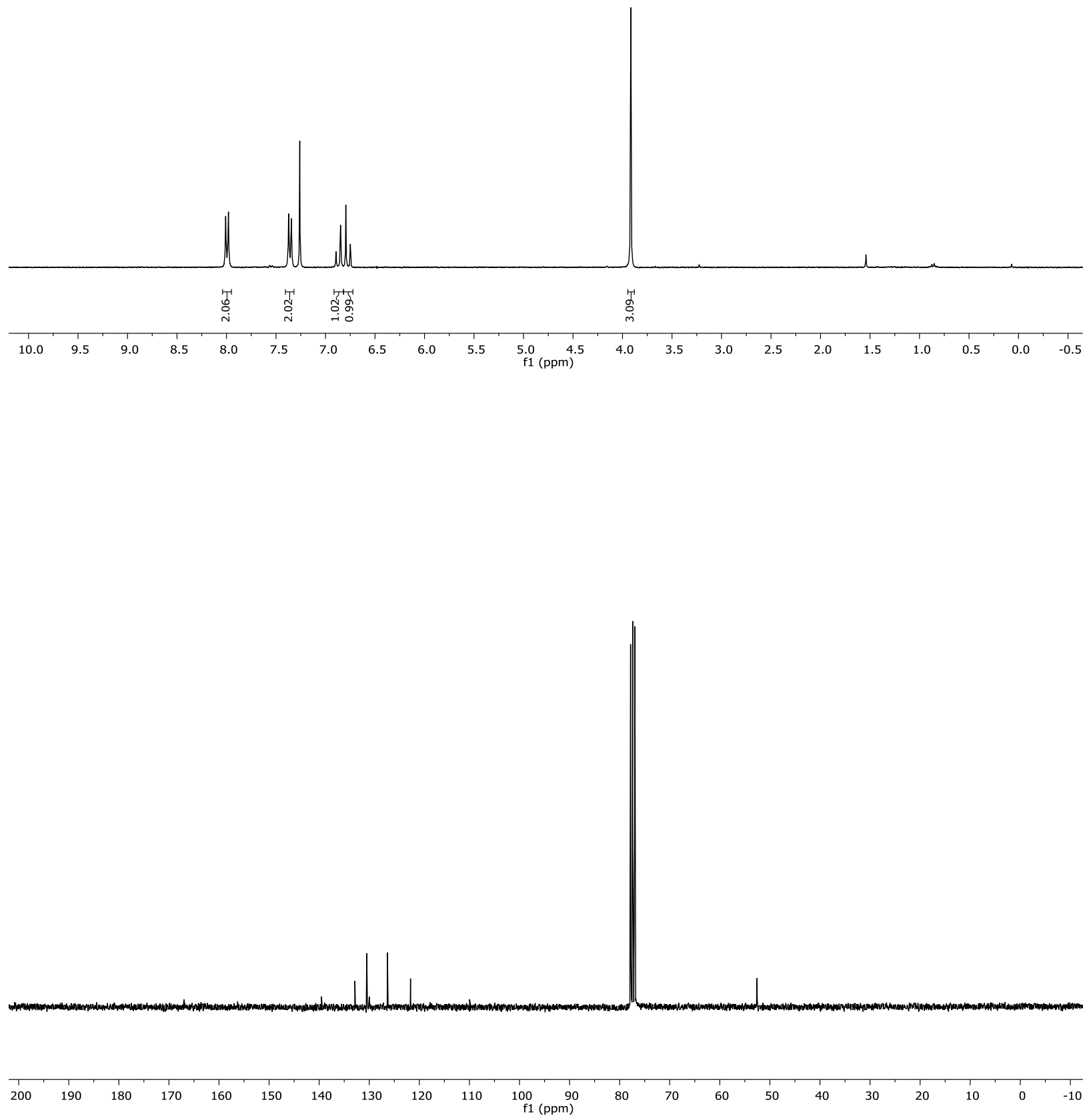


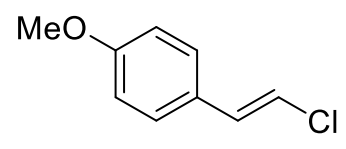

$10 \mathrm{e}$
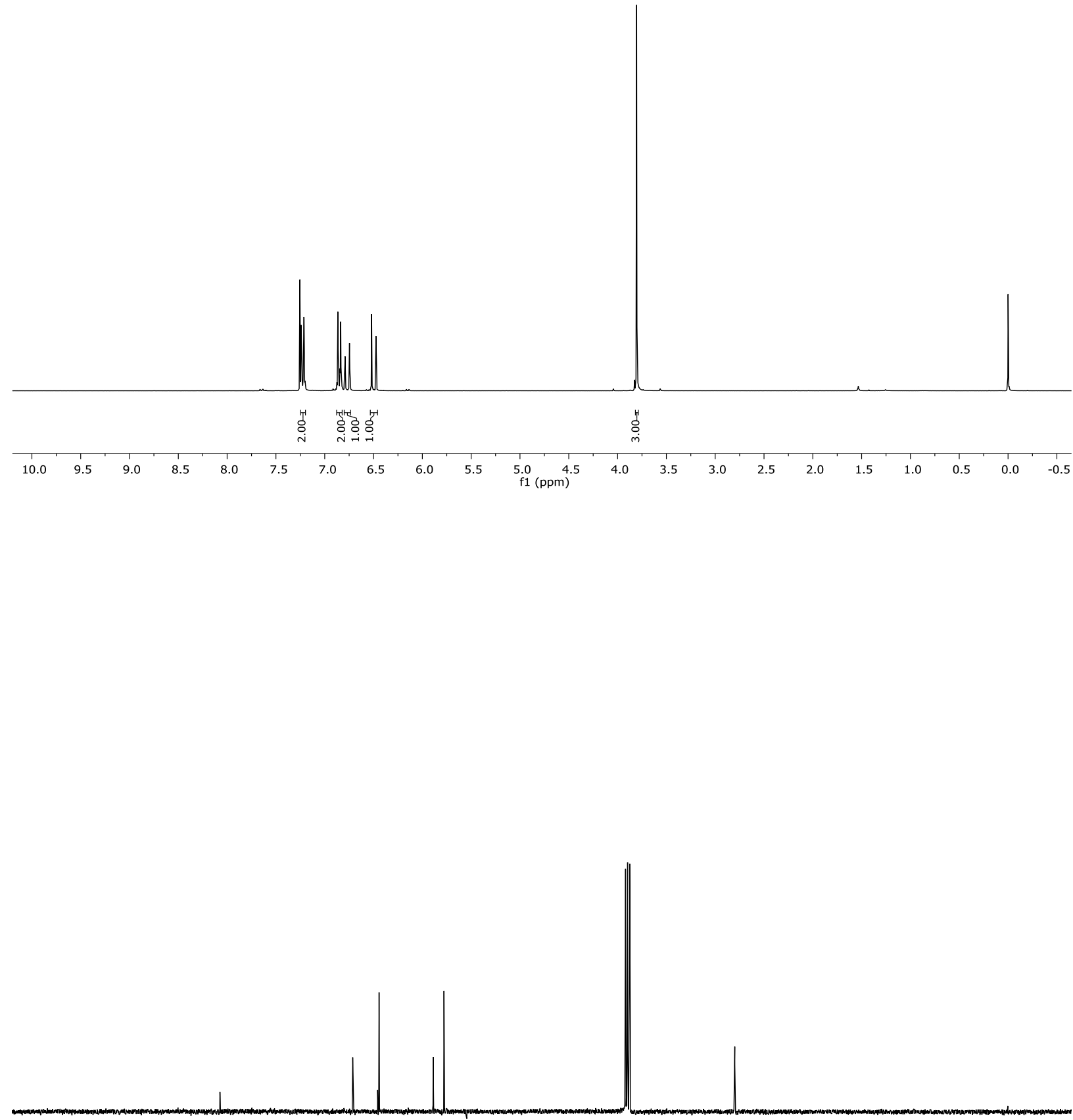

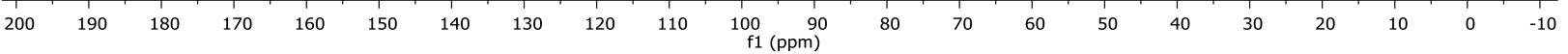




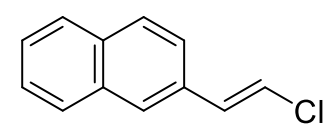

$10 f$

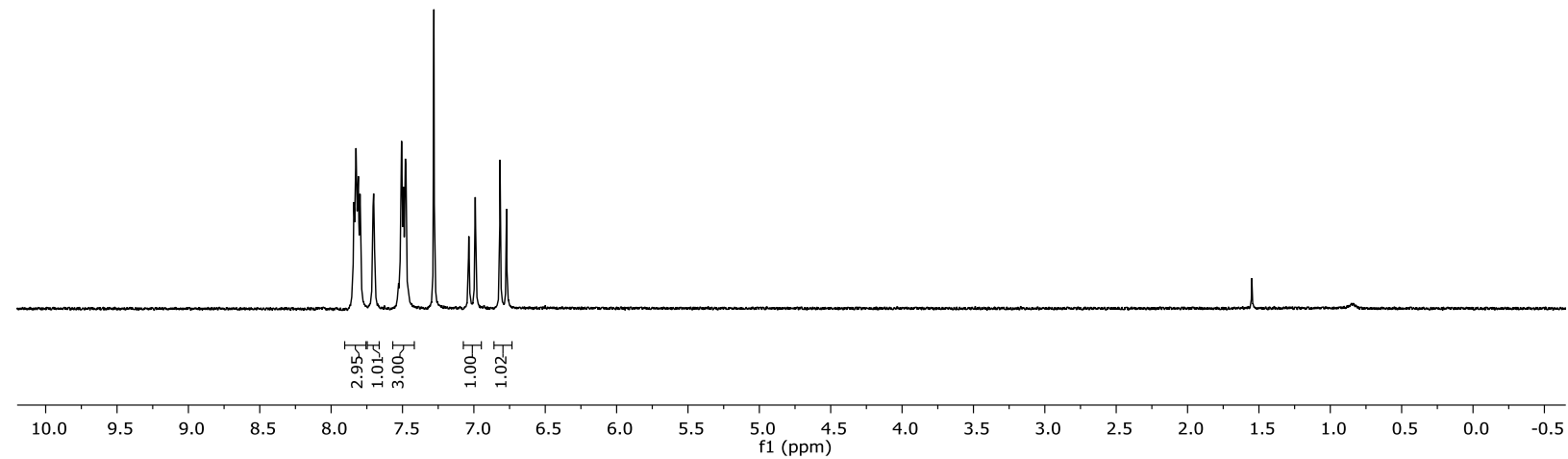

.

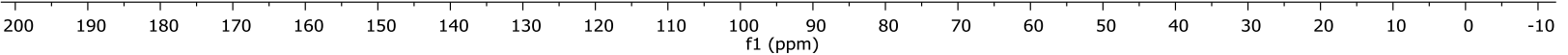




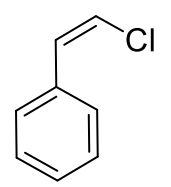

$10 \mathrm{~g}$
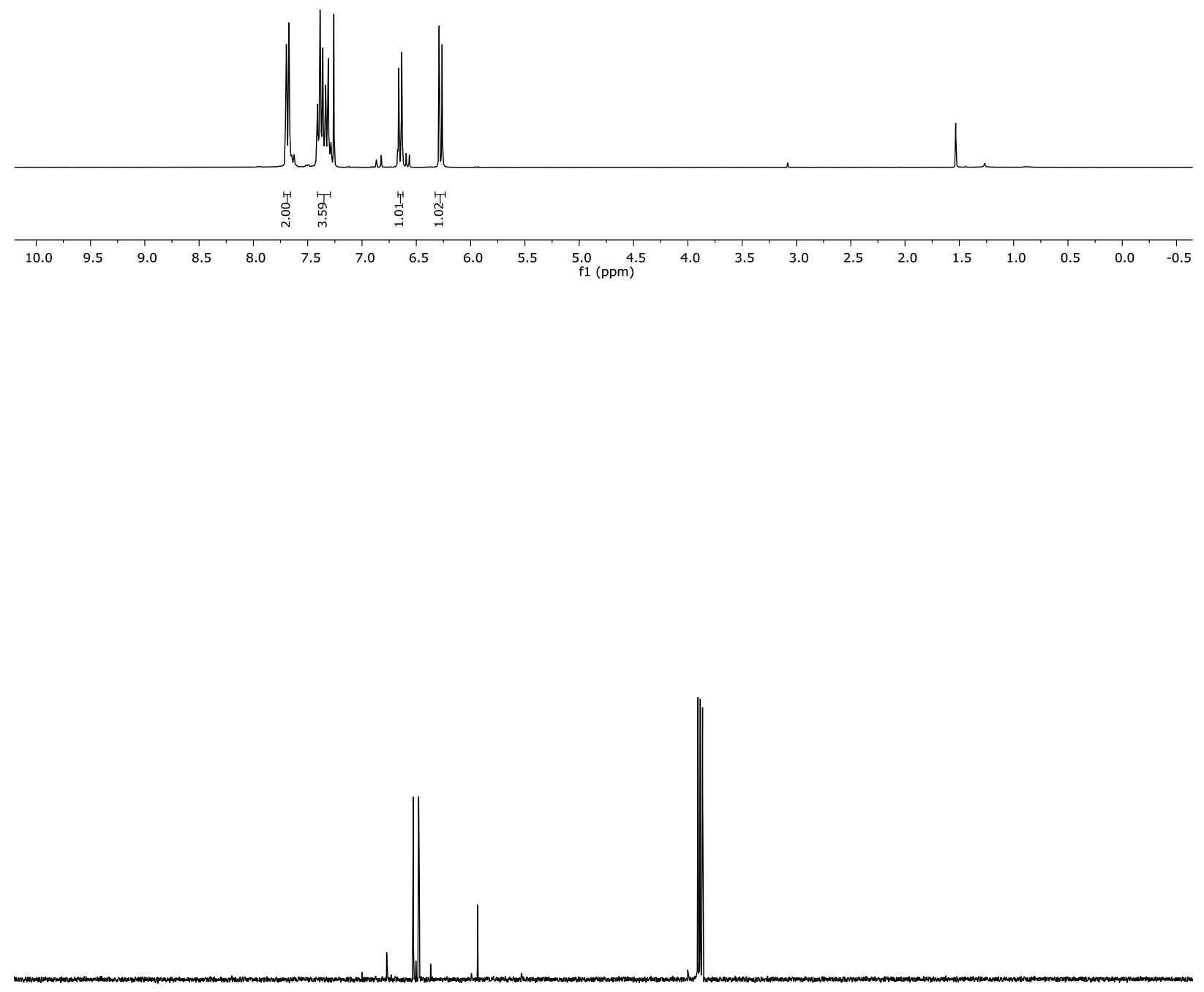

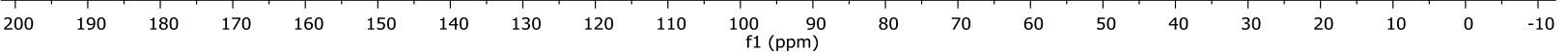




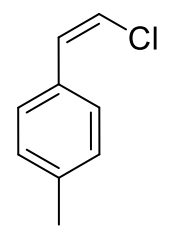

$10 \mathrm{~h}$
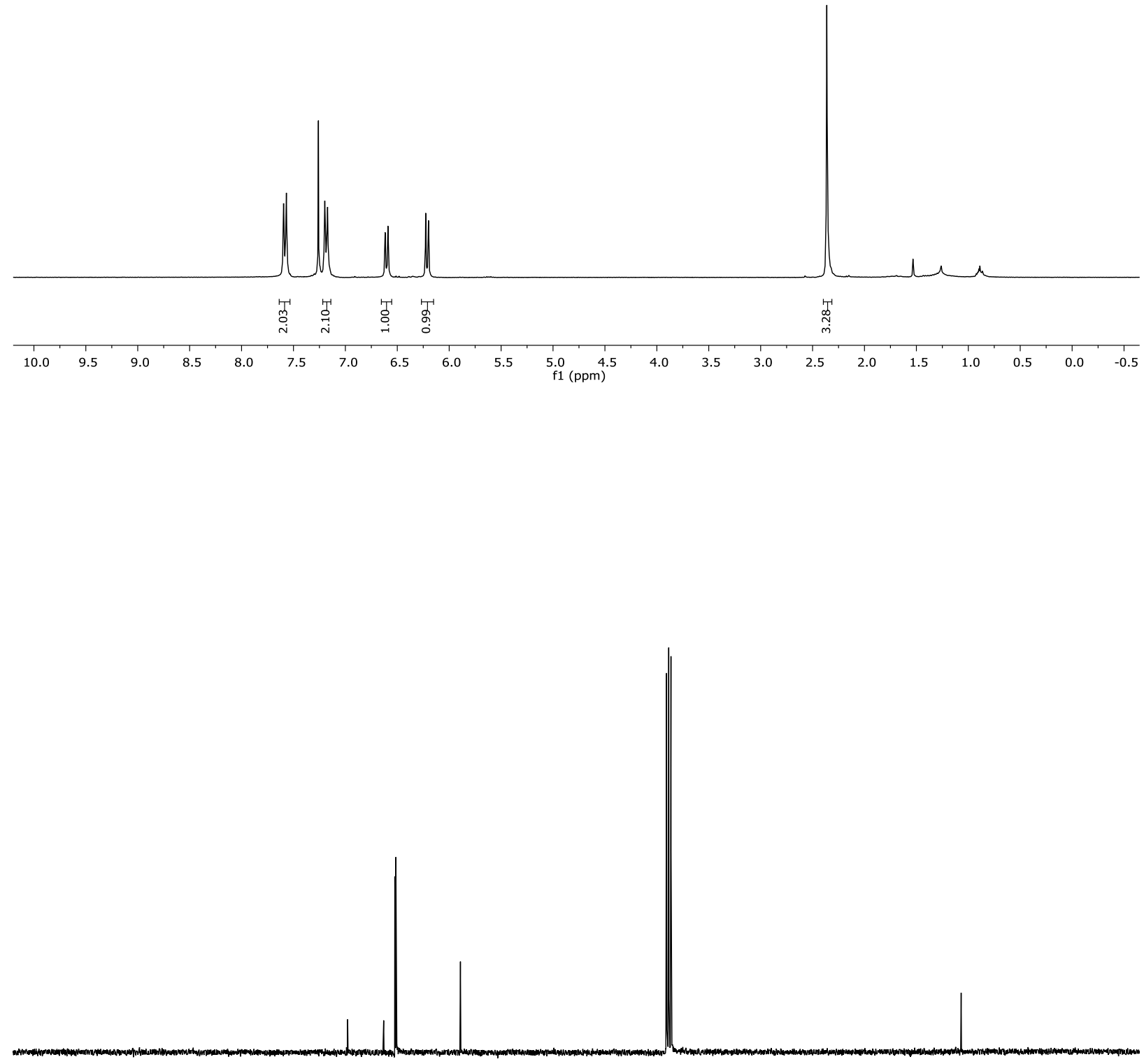

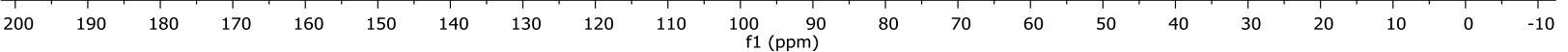




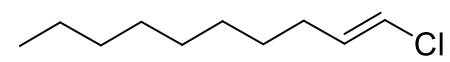

$10 \mathbf{i}$
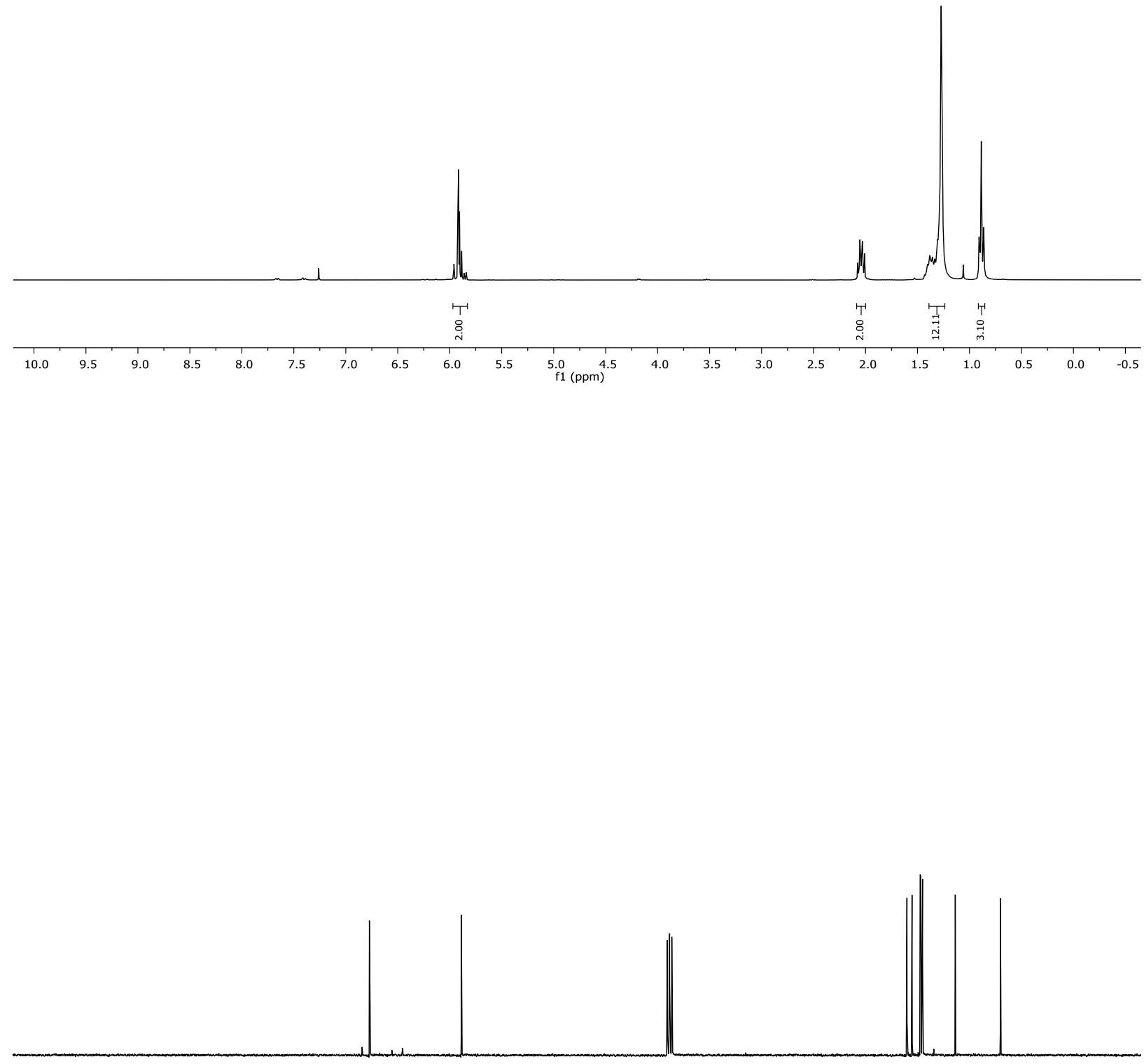


\section{TBDPSO $\frown \mathrm{Cl}$}

$10 \mathrm{j}$
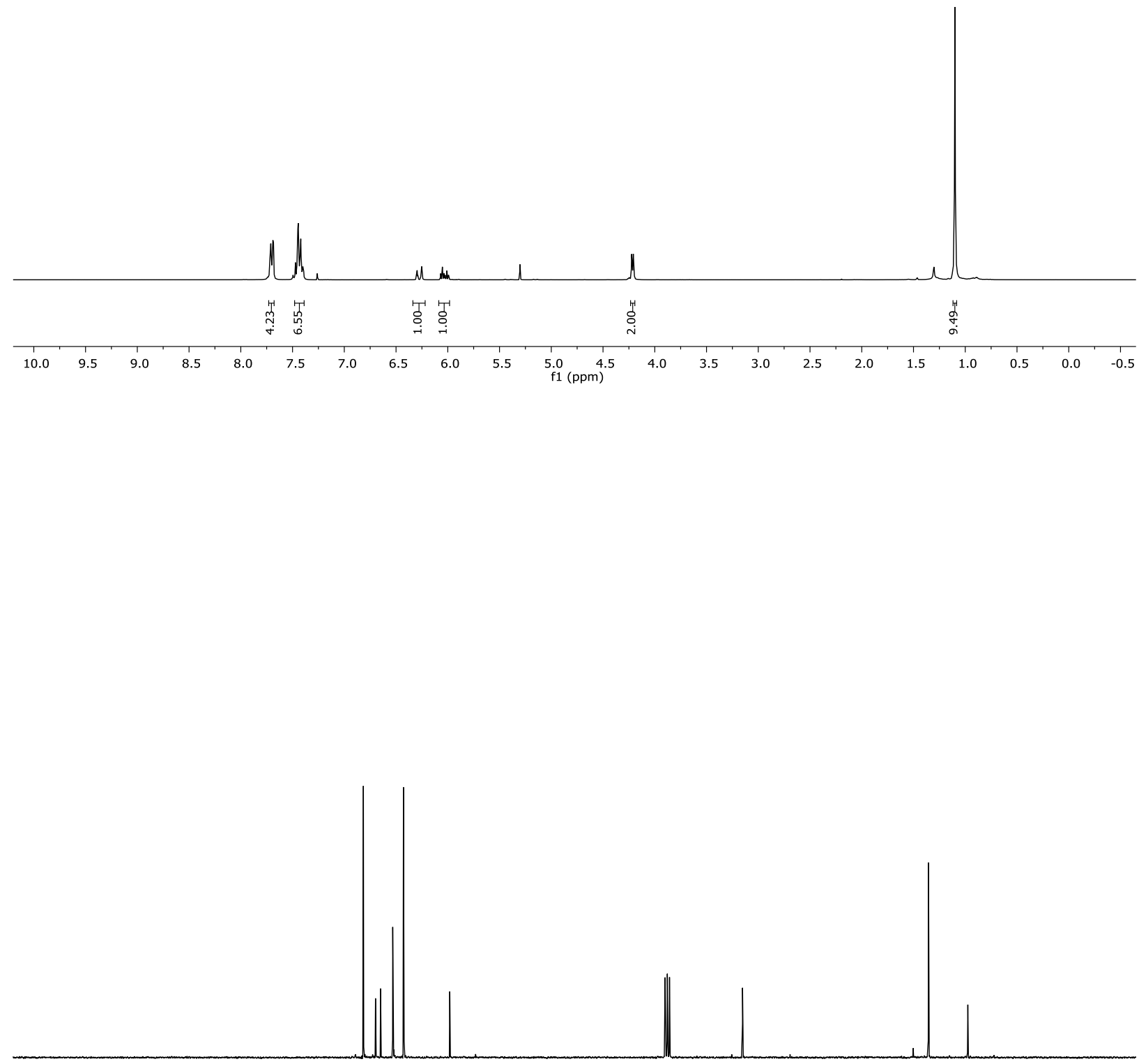

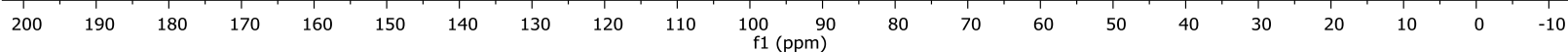




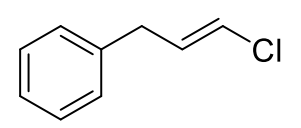

$10 \mathrm{k}$
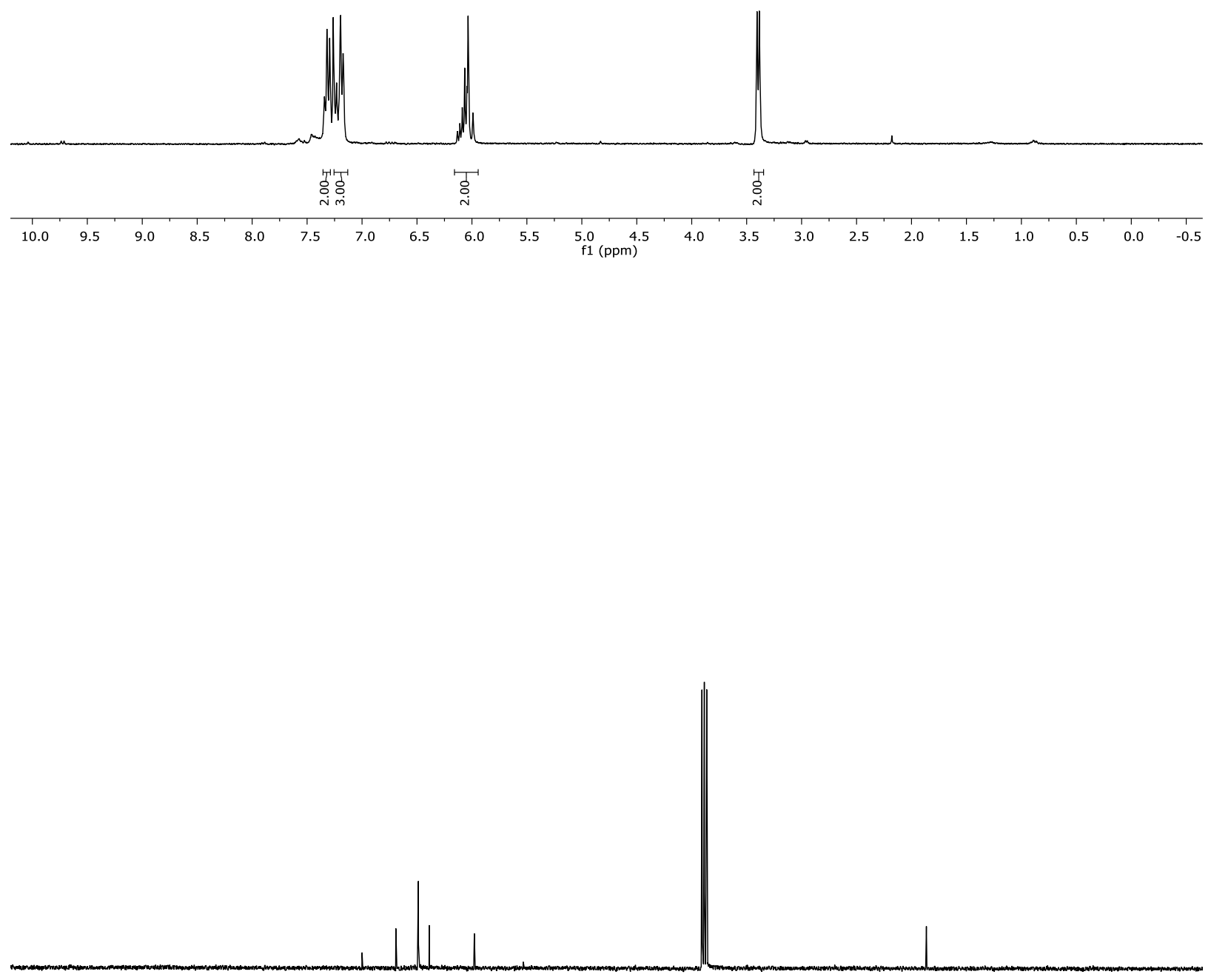

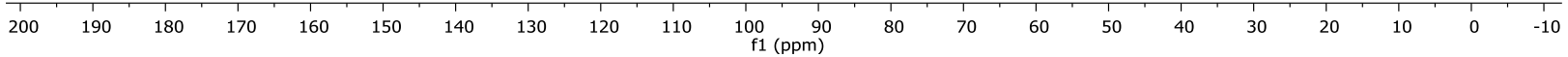




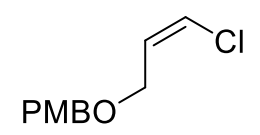

101
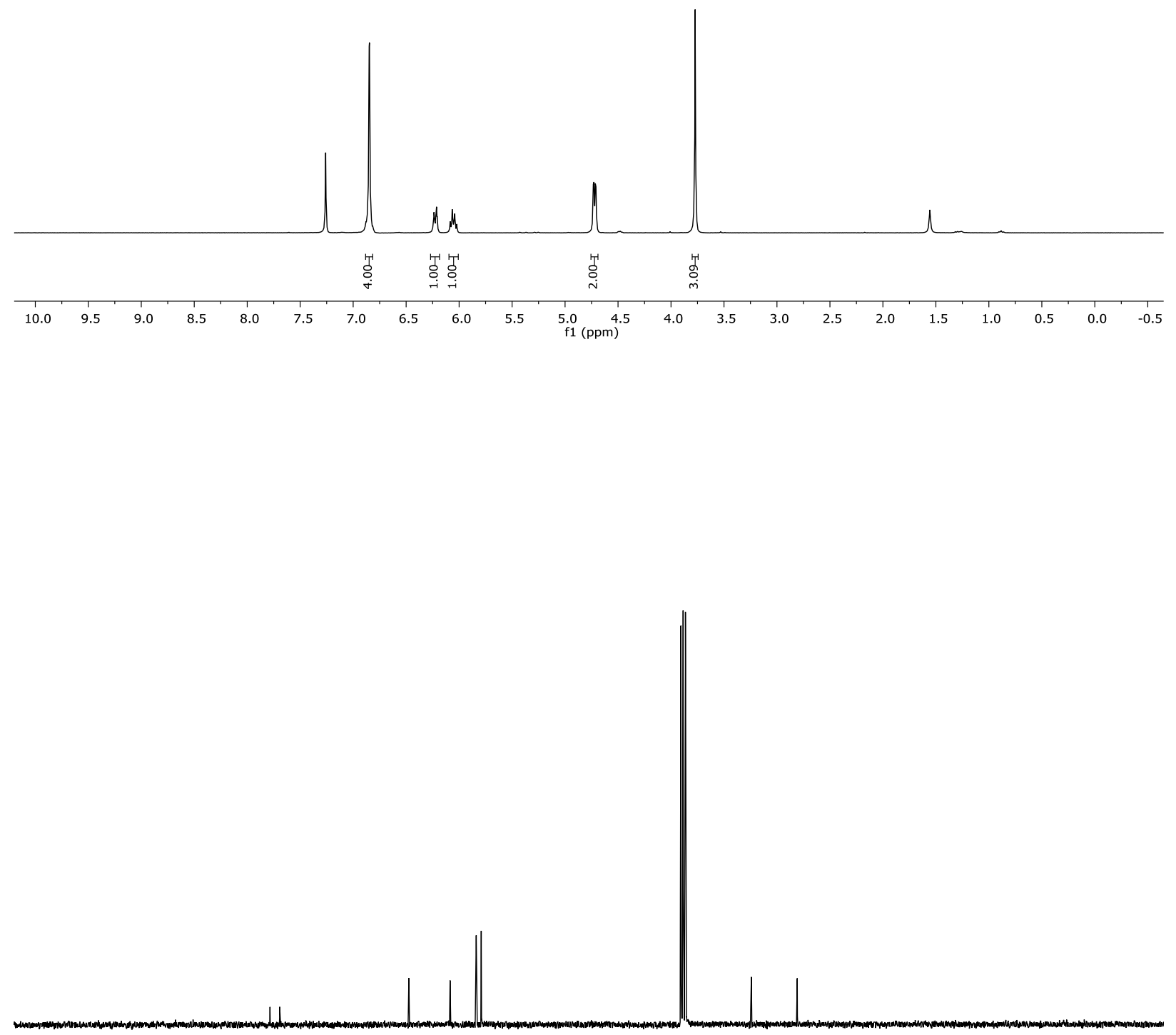

$\begin{array}{llll}190 & 180 & 170 & 160\end{array}$

$\begin{array}{cccccc}150 & 140 & 130 & 120 & 110 & 100 \begin{array}{c}90 \\ \mathrm{f} 1(\mathrm{ppm})\end{array}\end{array}$

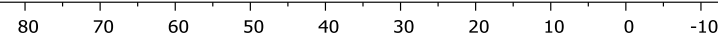




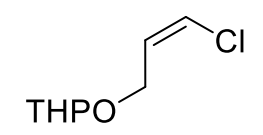

$10 \mathrm{~m}$
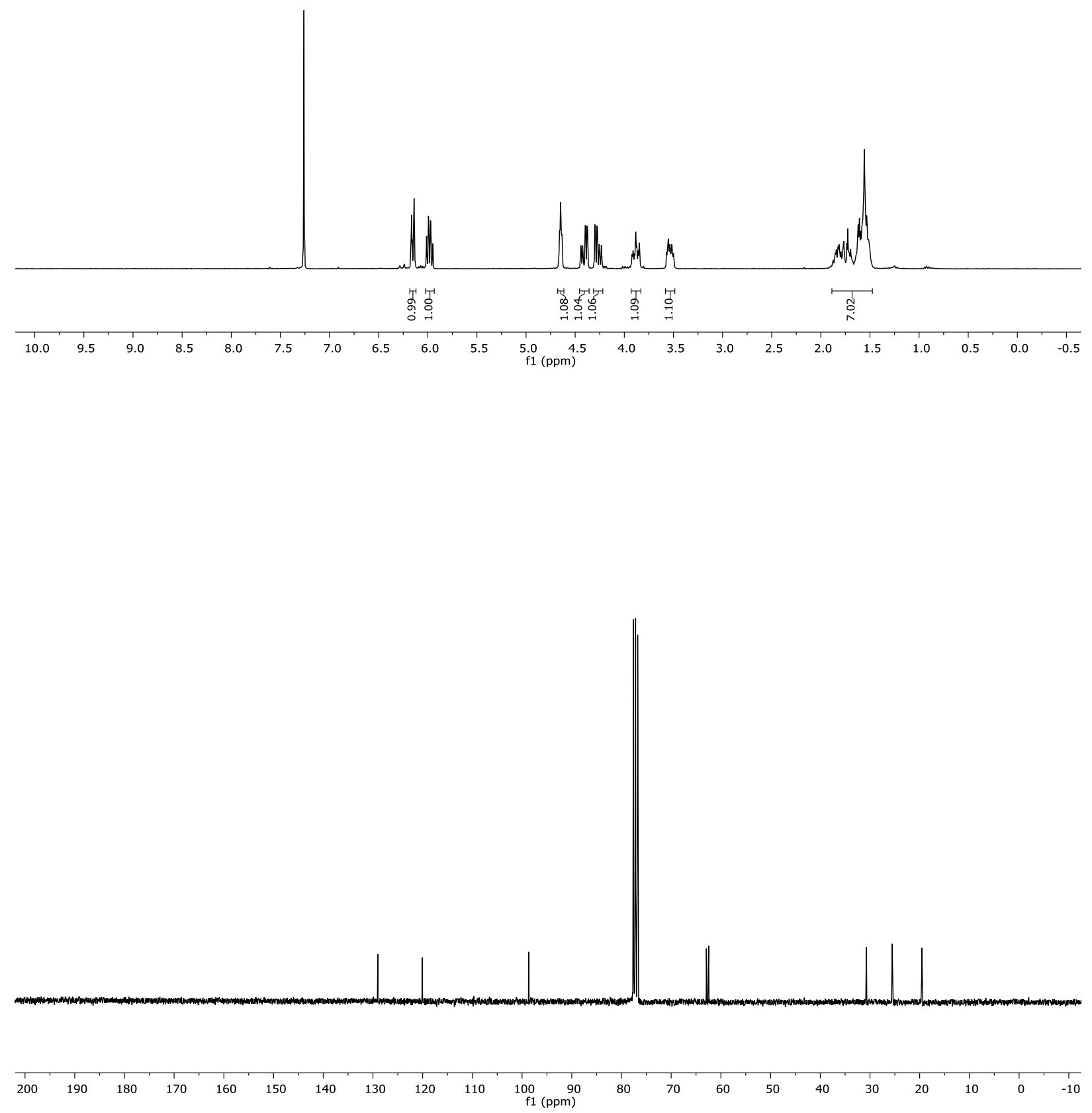

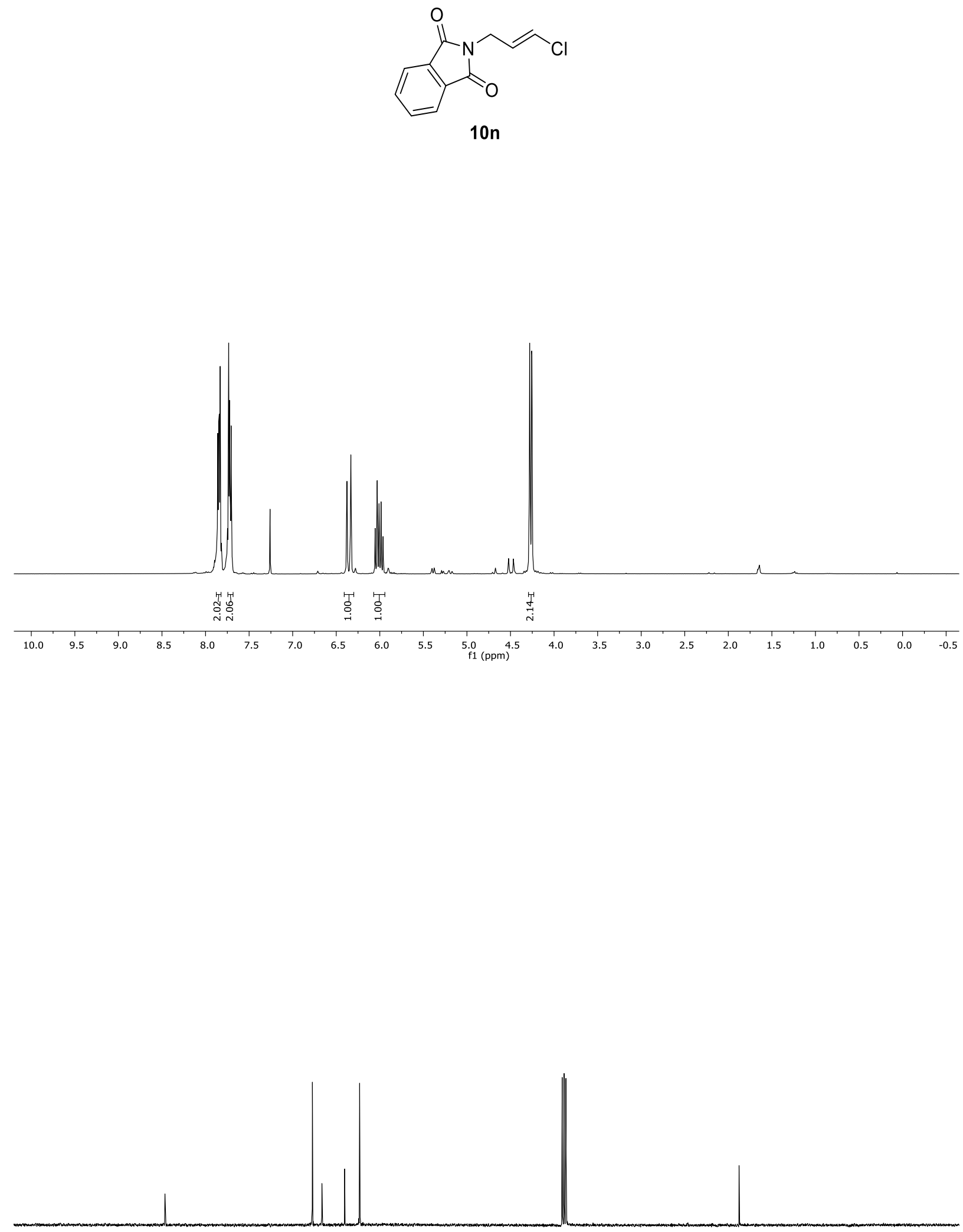

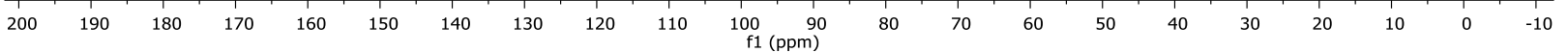



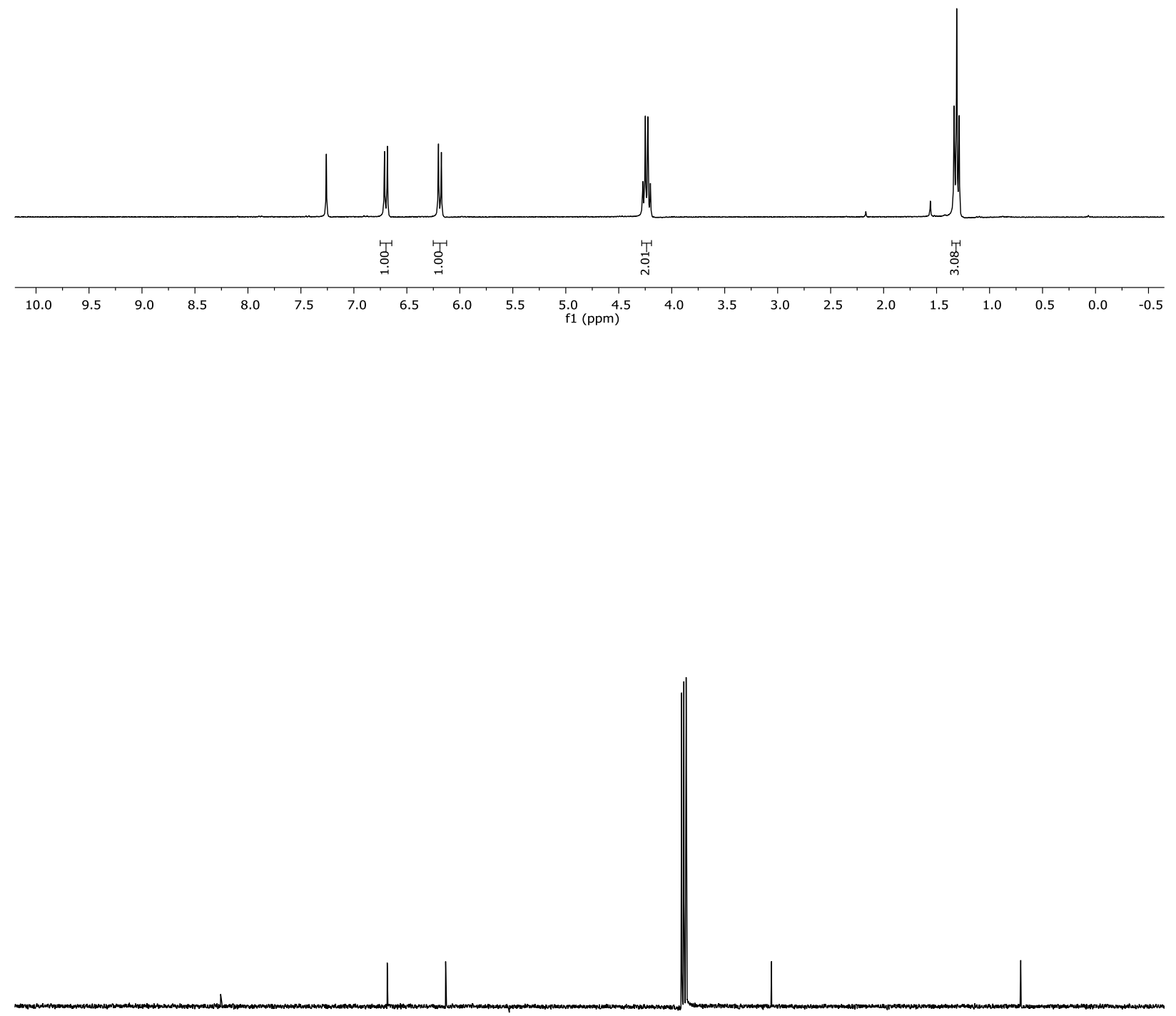

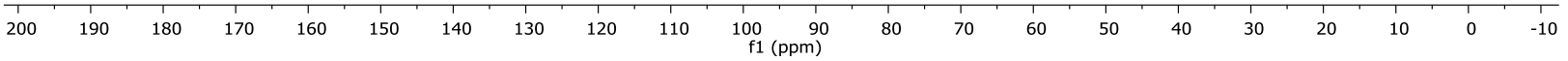




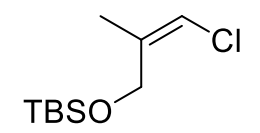

$10 p$
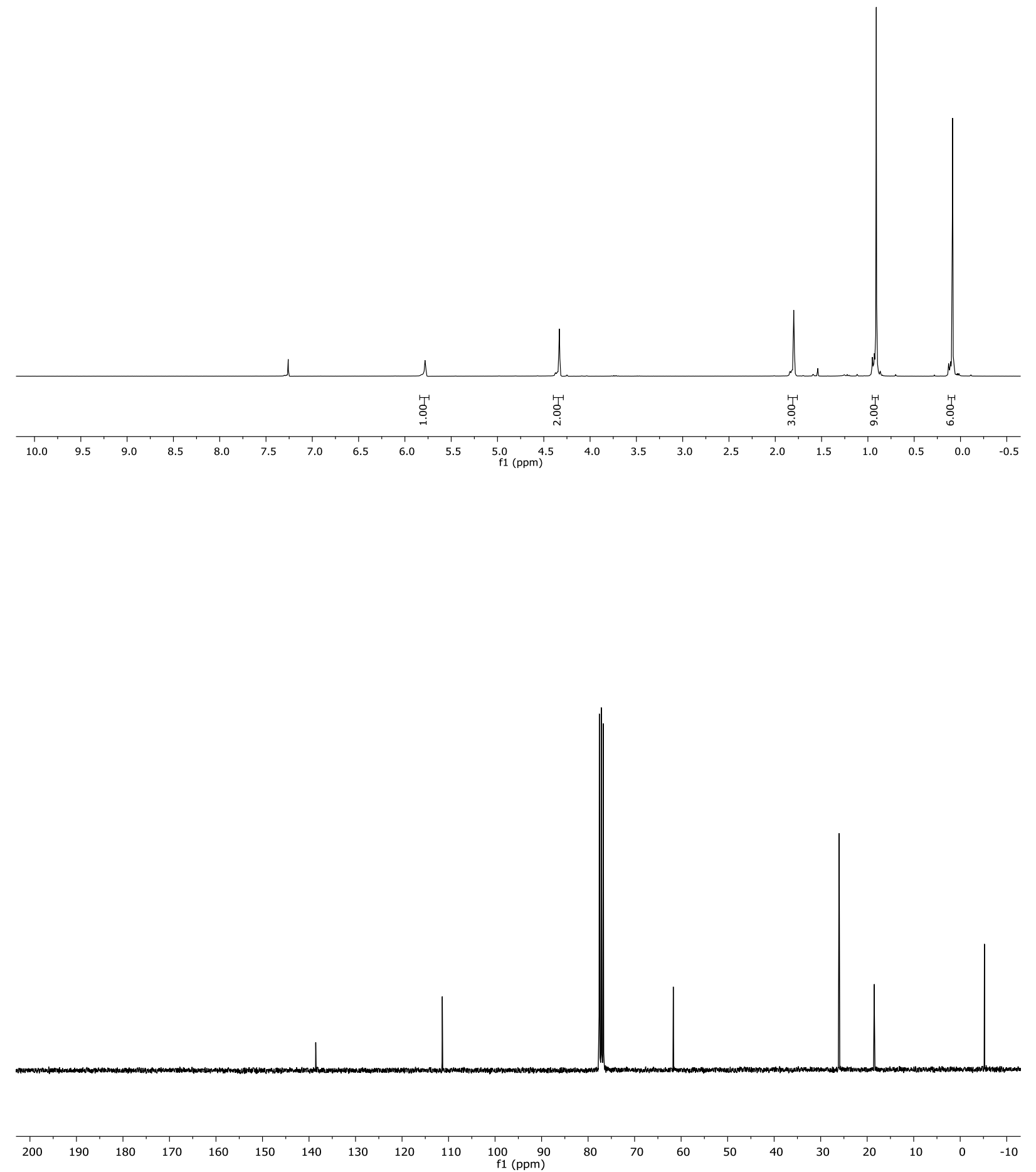


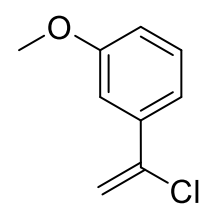

$10 q$
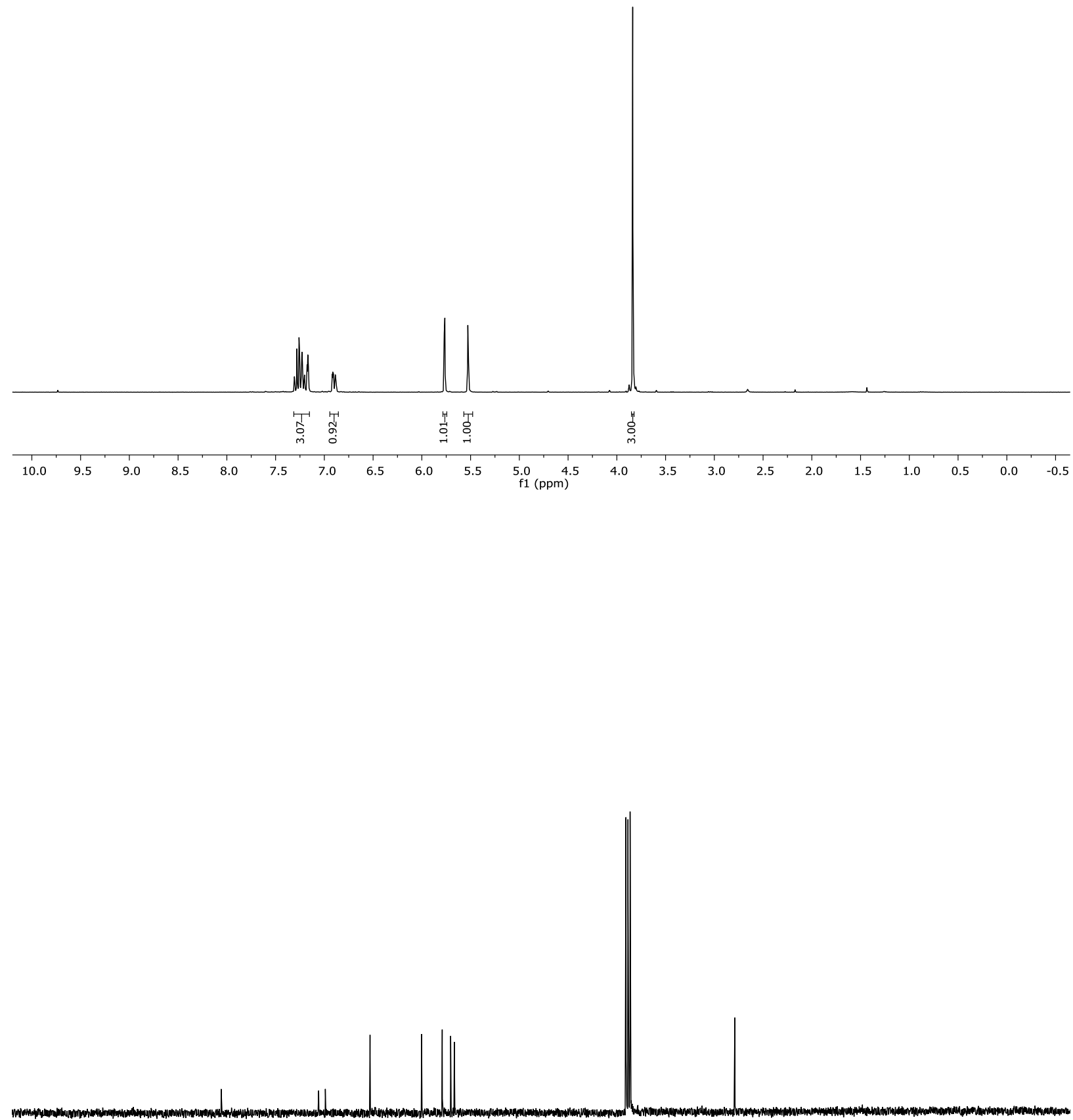

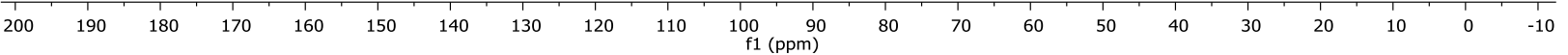




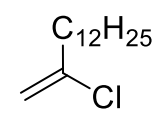

$10 r$
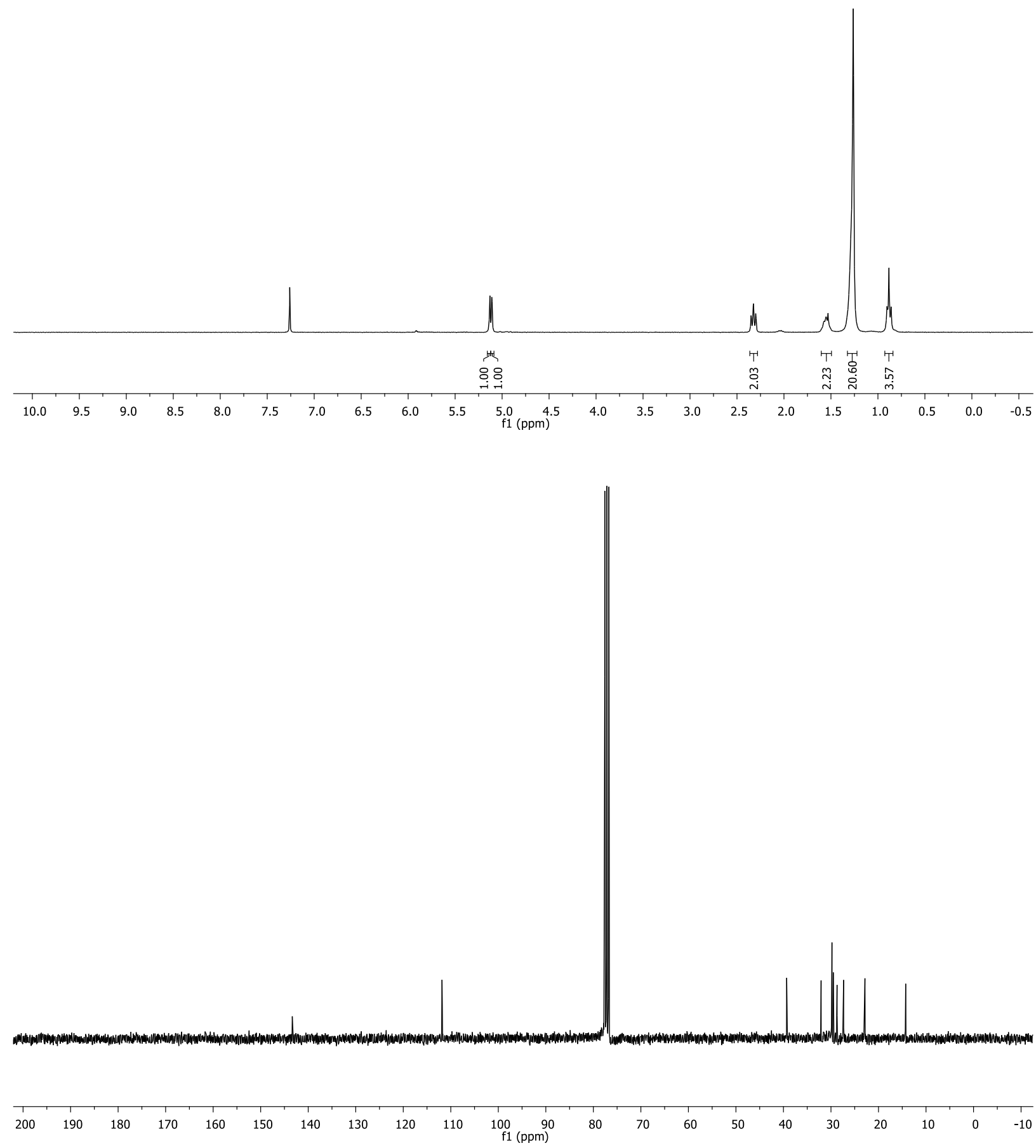
TBSO<smiles>C=C(Cl)CC</smiles>

10s
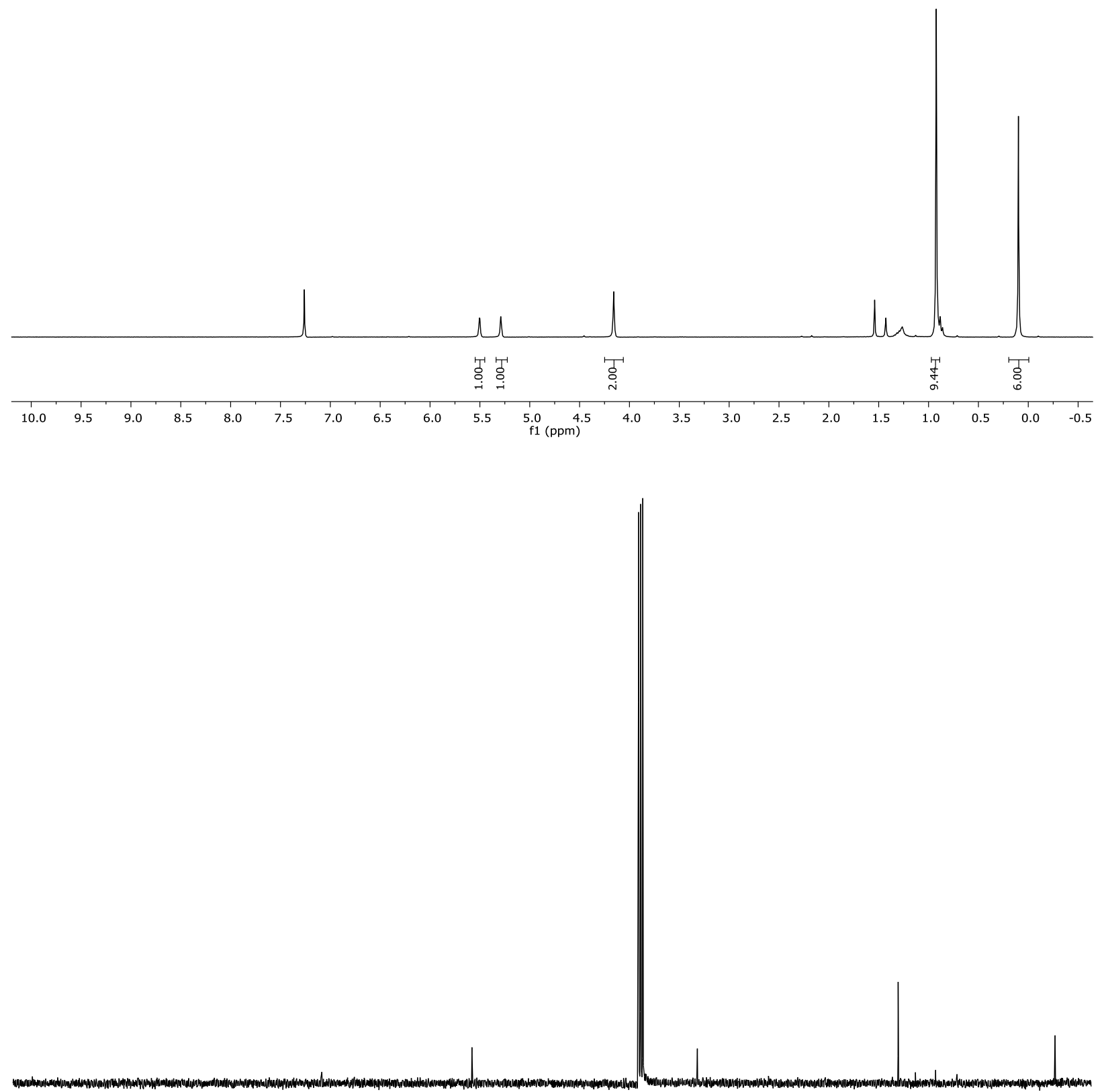

$\begin{array}{lllllllllllllllllllllll}200 & 190 & 180 & 170 & 160 & 150 & 140 & 130 & 120 & 110 & 100 & 90 & 80 & 70 & 60 & 50 & 40 & 30 & 20 & 10 & 0 & -10\end{array}$




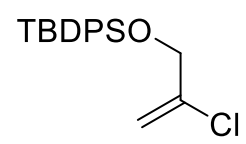

$10 t$
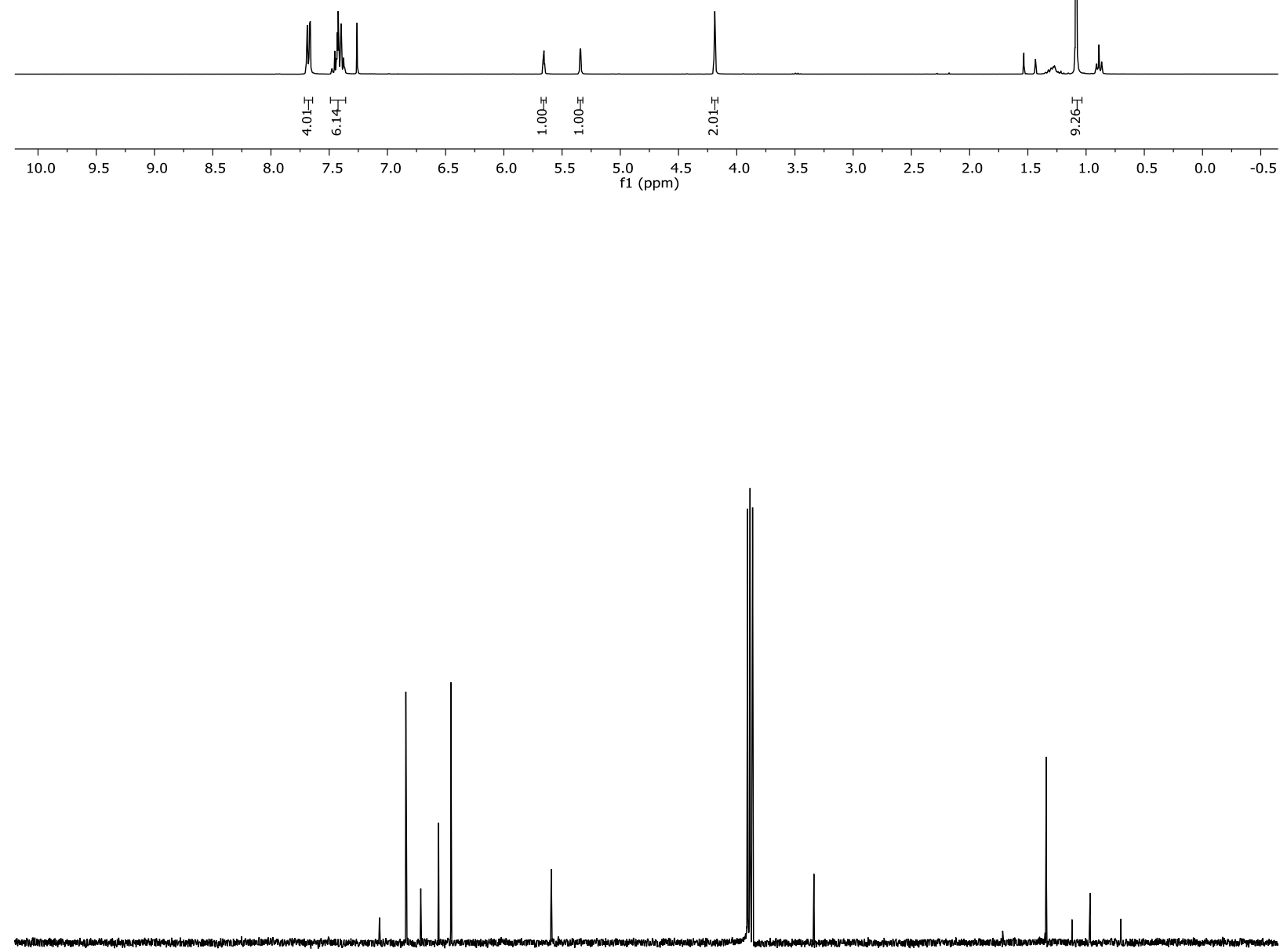

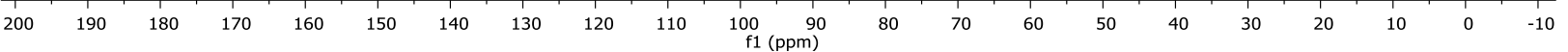




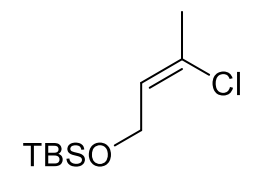

10u
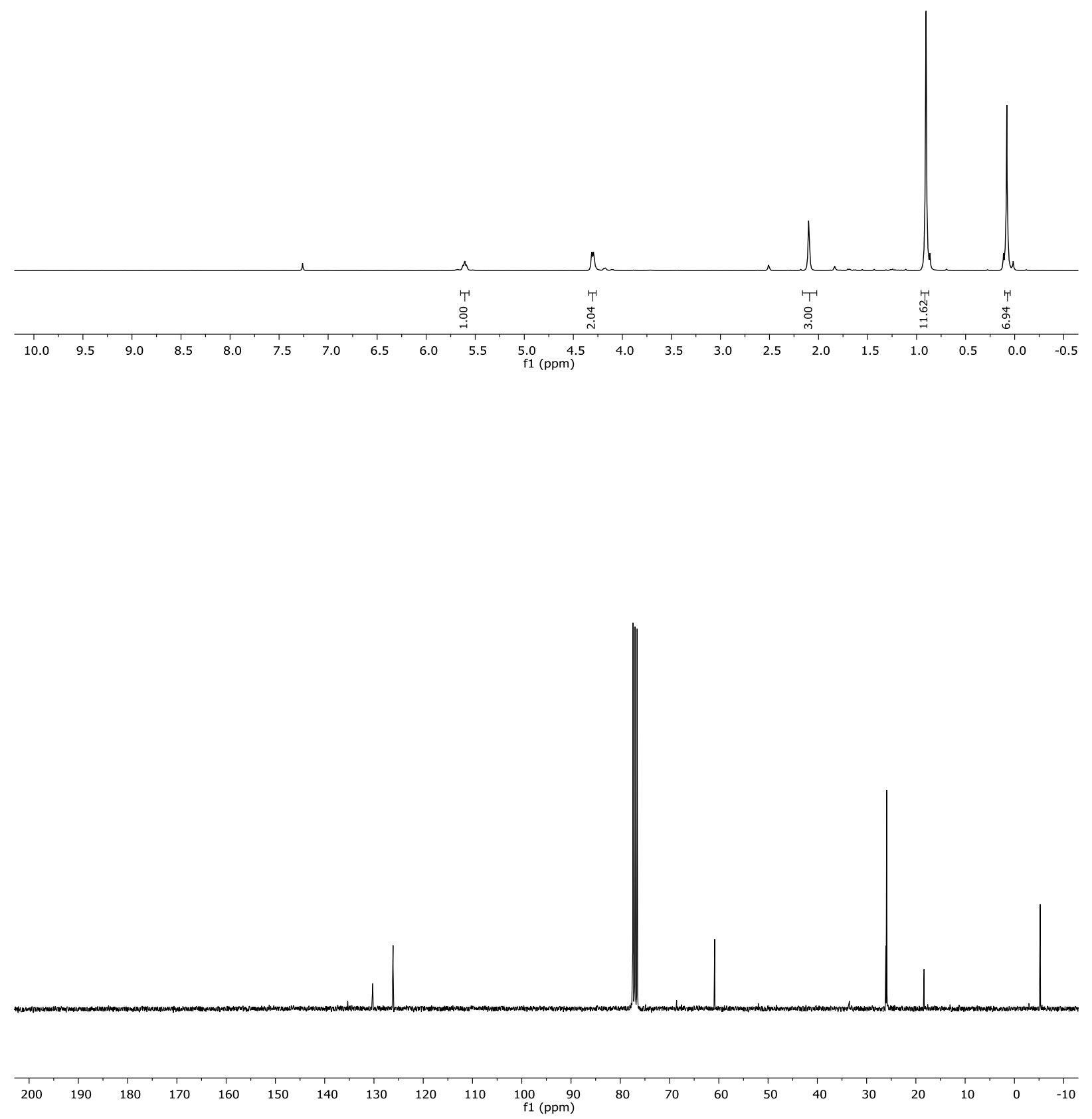

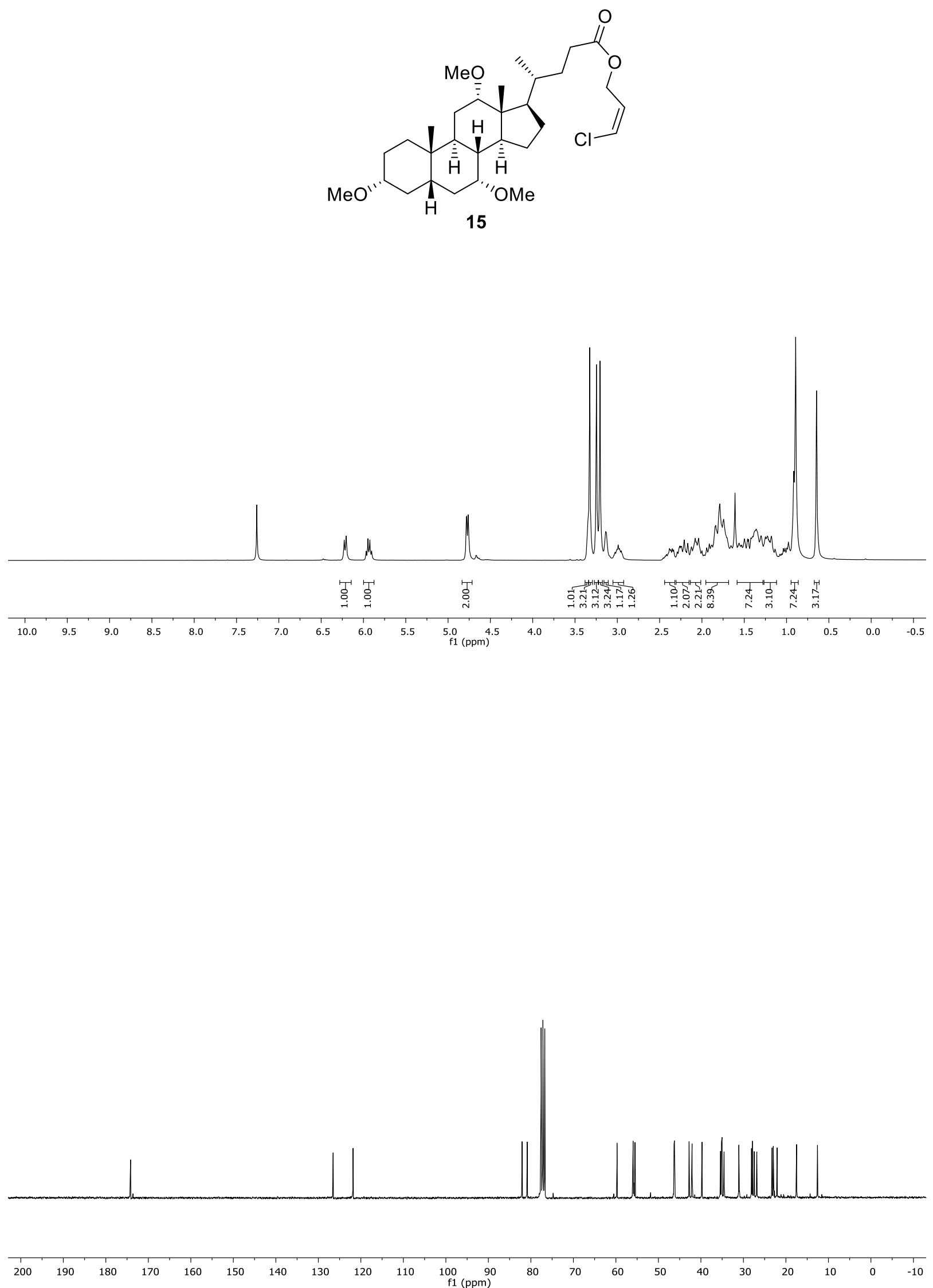


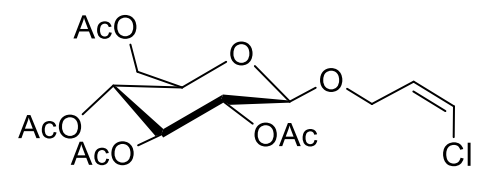

17
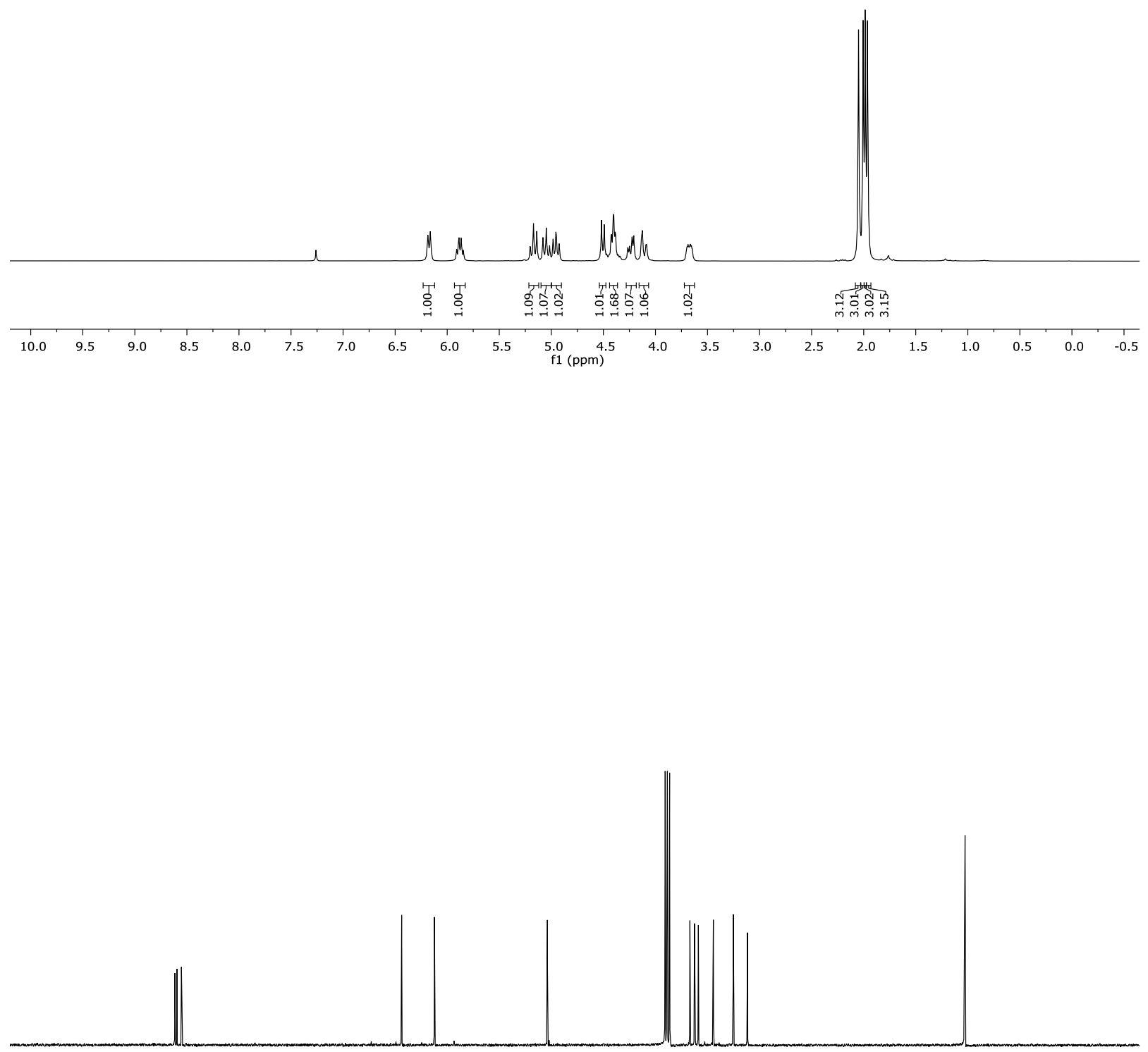

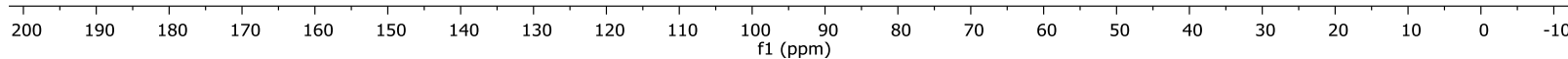


<smiles>O=c1[nH]c(=O)n(C2CC(O)C(CO)O2)cc1C=CCl</smiles>

19
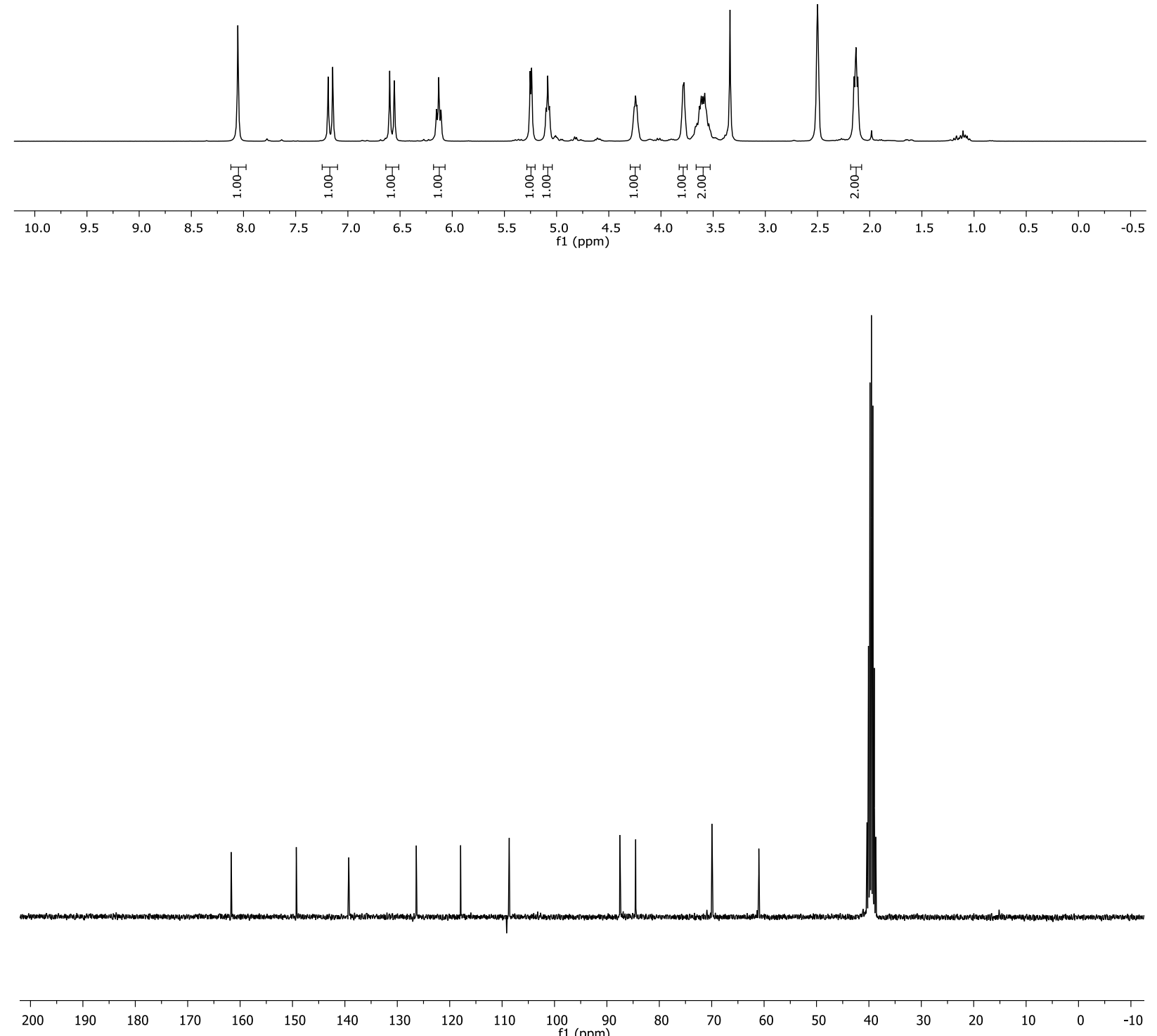


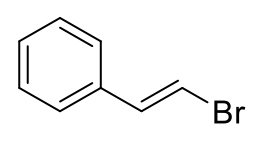

$11 \mathrm{a}$
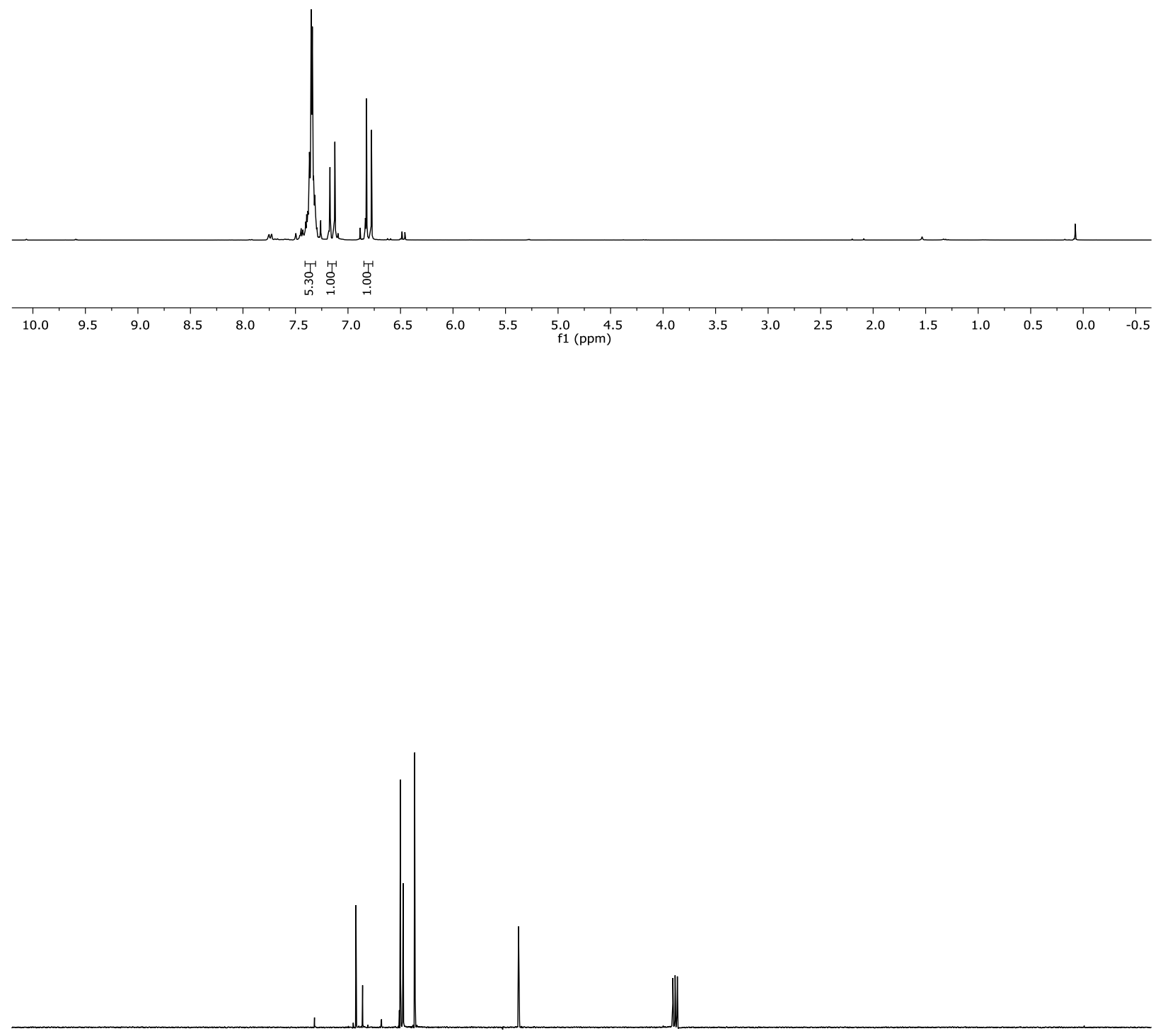

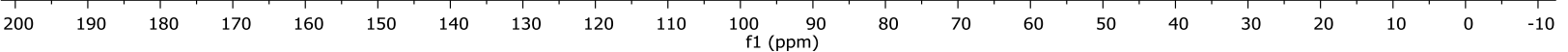




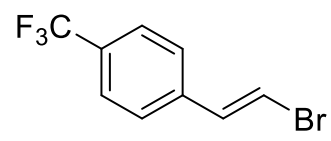

$11 \mathrm{~b}$
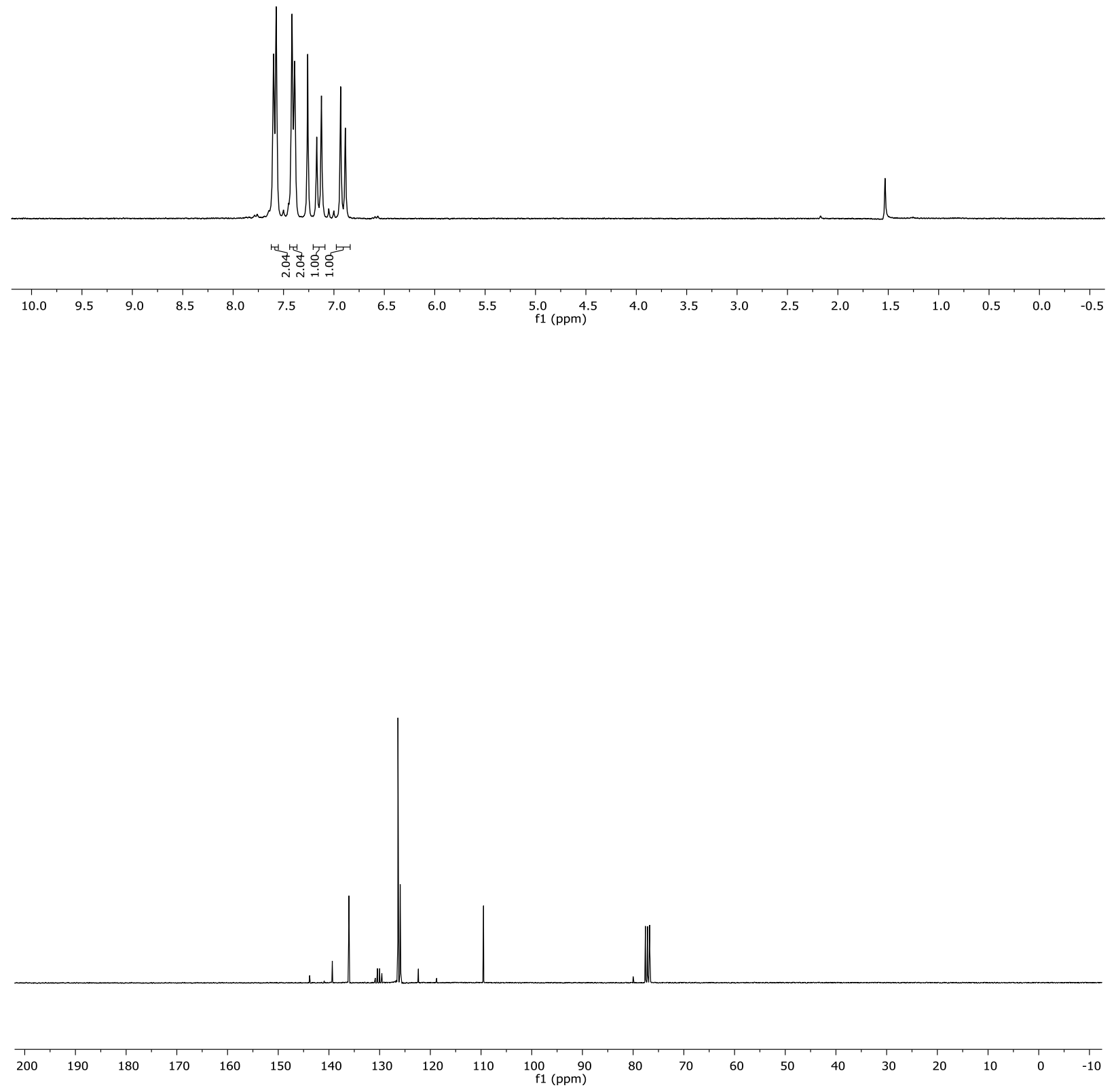


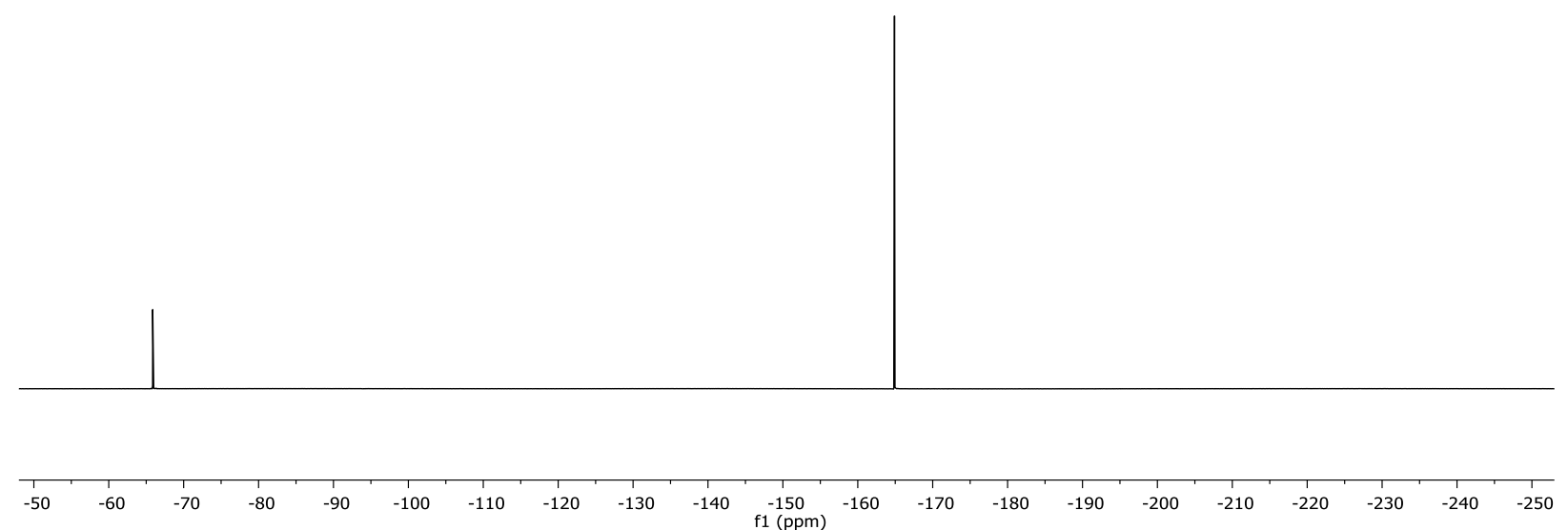




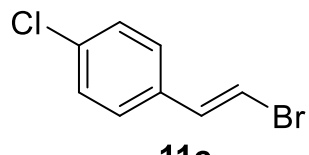

$11 \mathrm{c}$
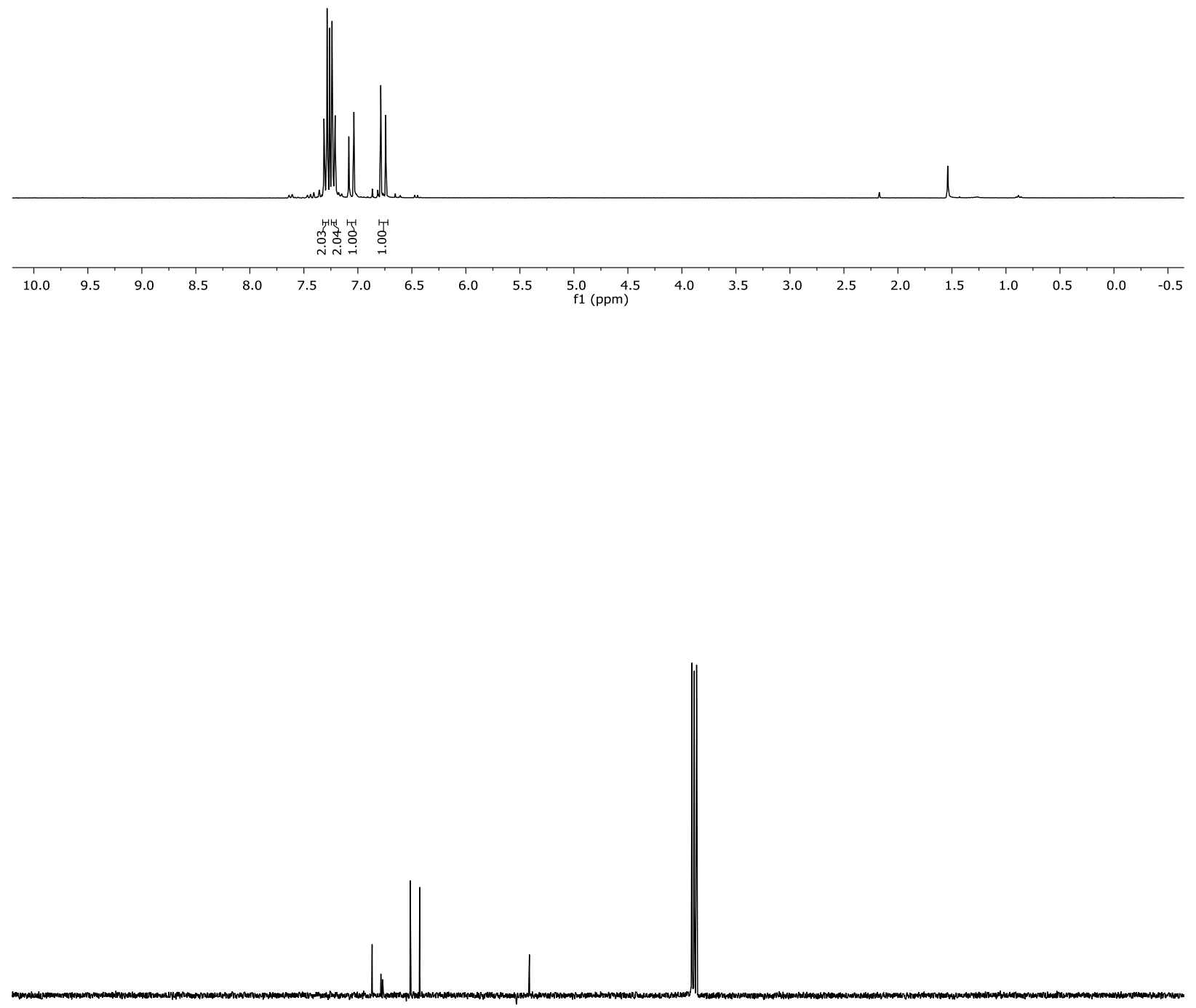

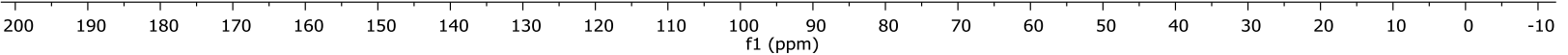




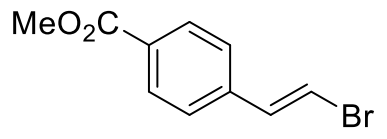

11d
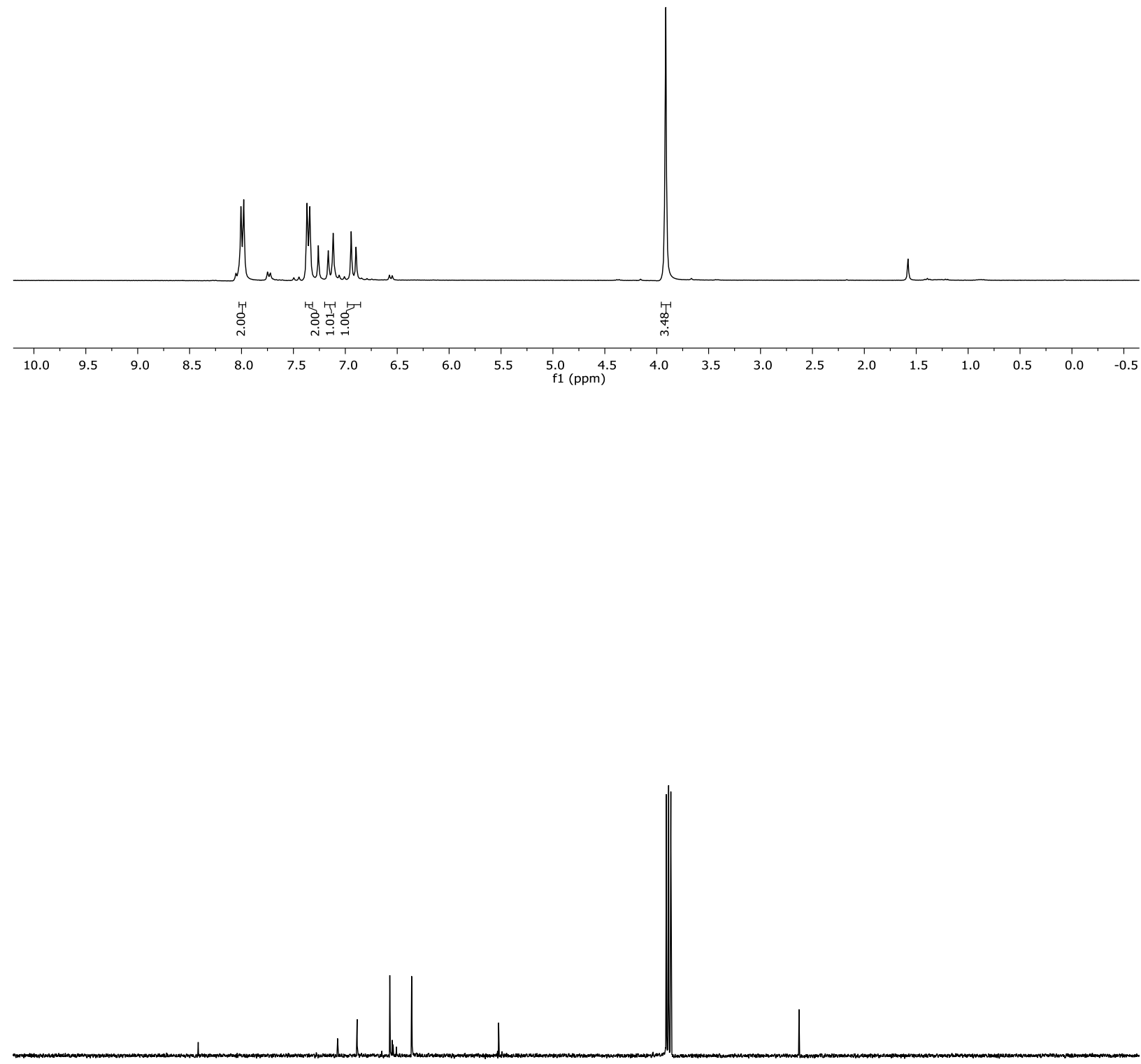

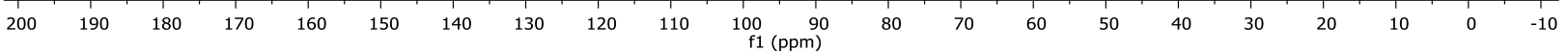




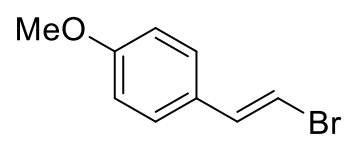

$11 \mathrm{e}$
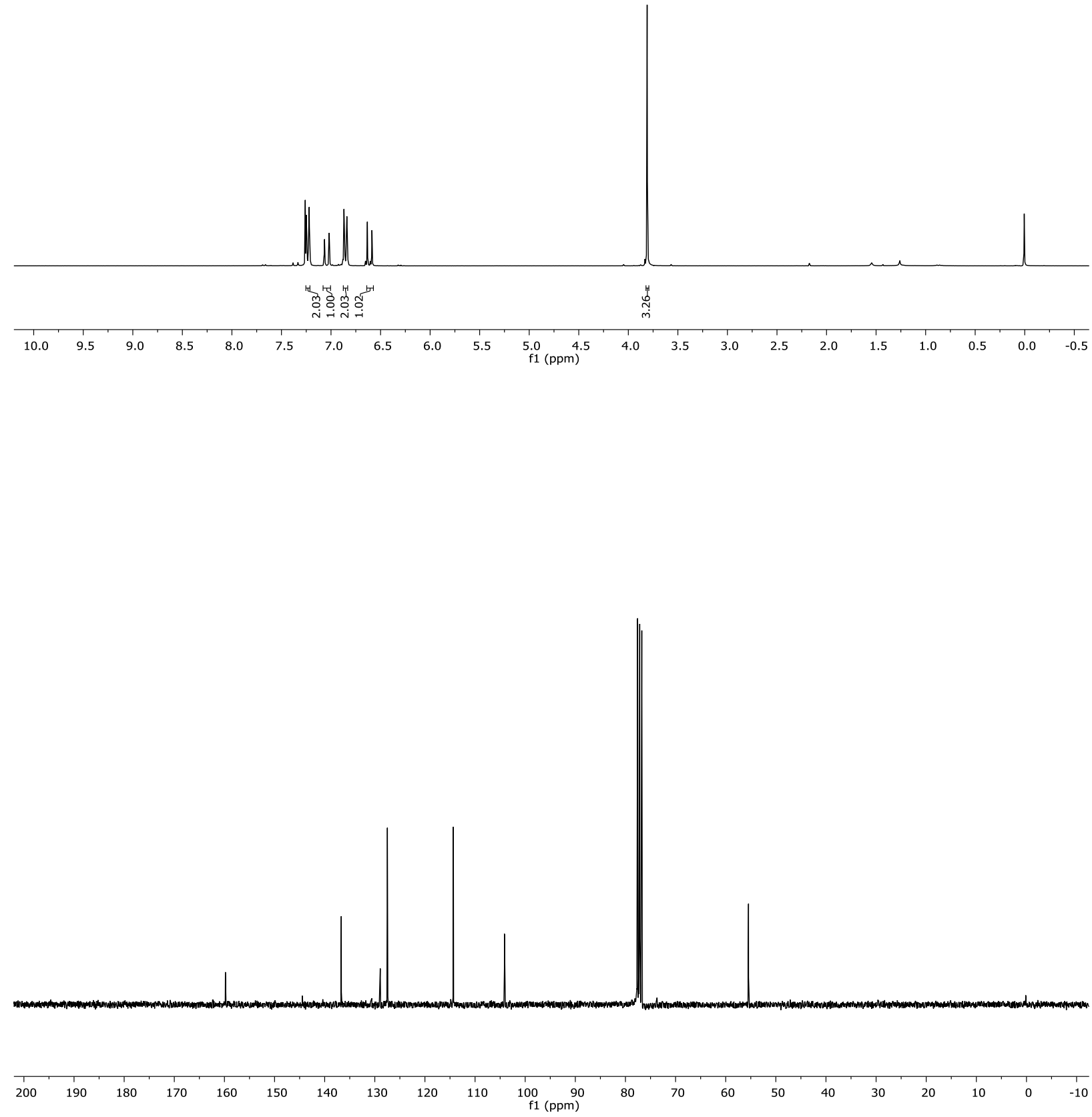


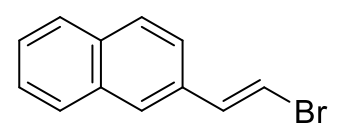

$11 f$
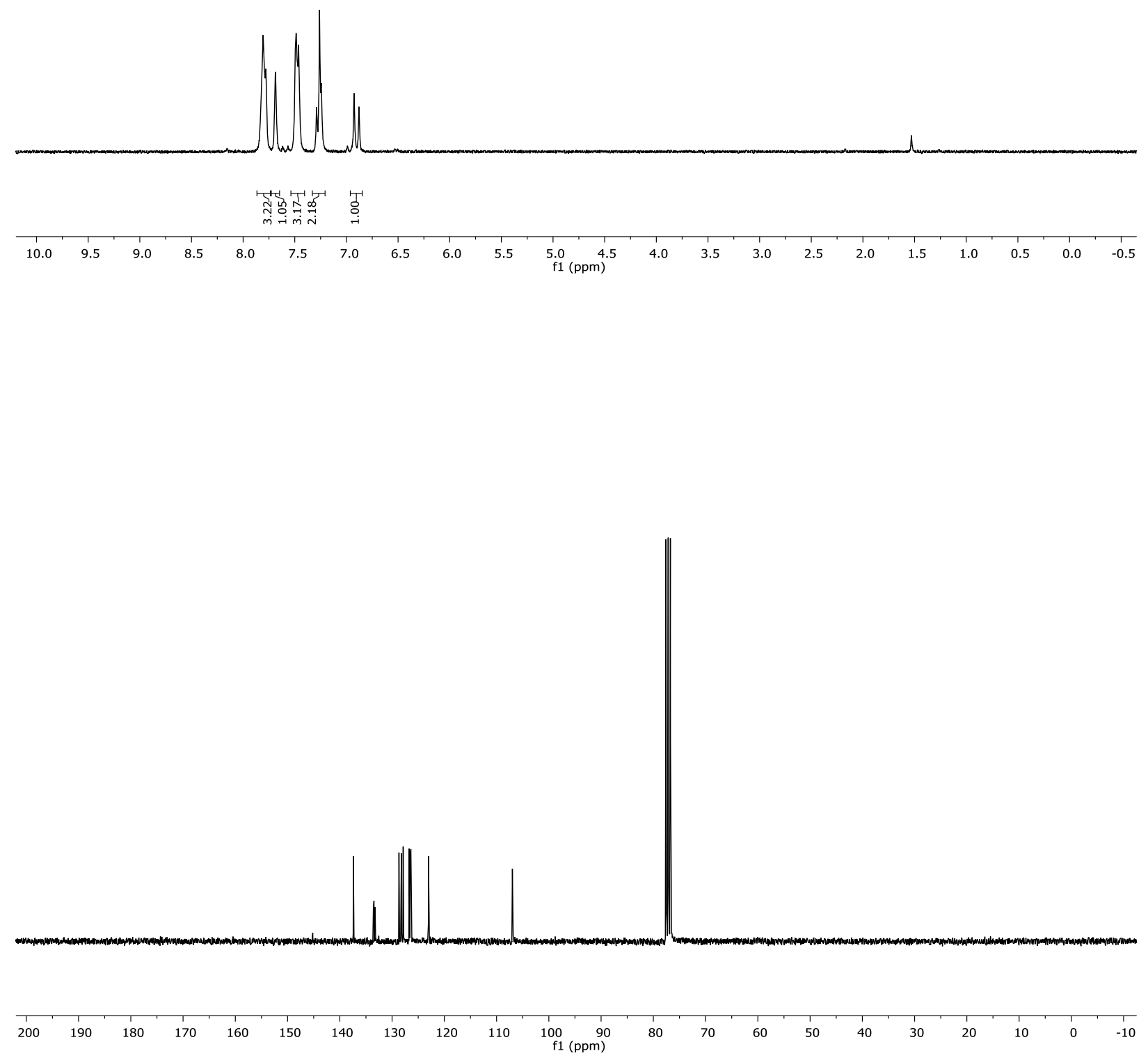


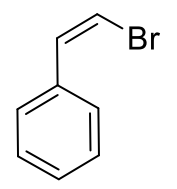

$11 \mathrm{~g}$
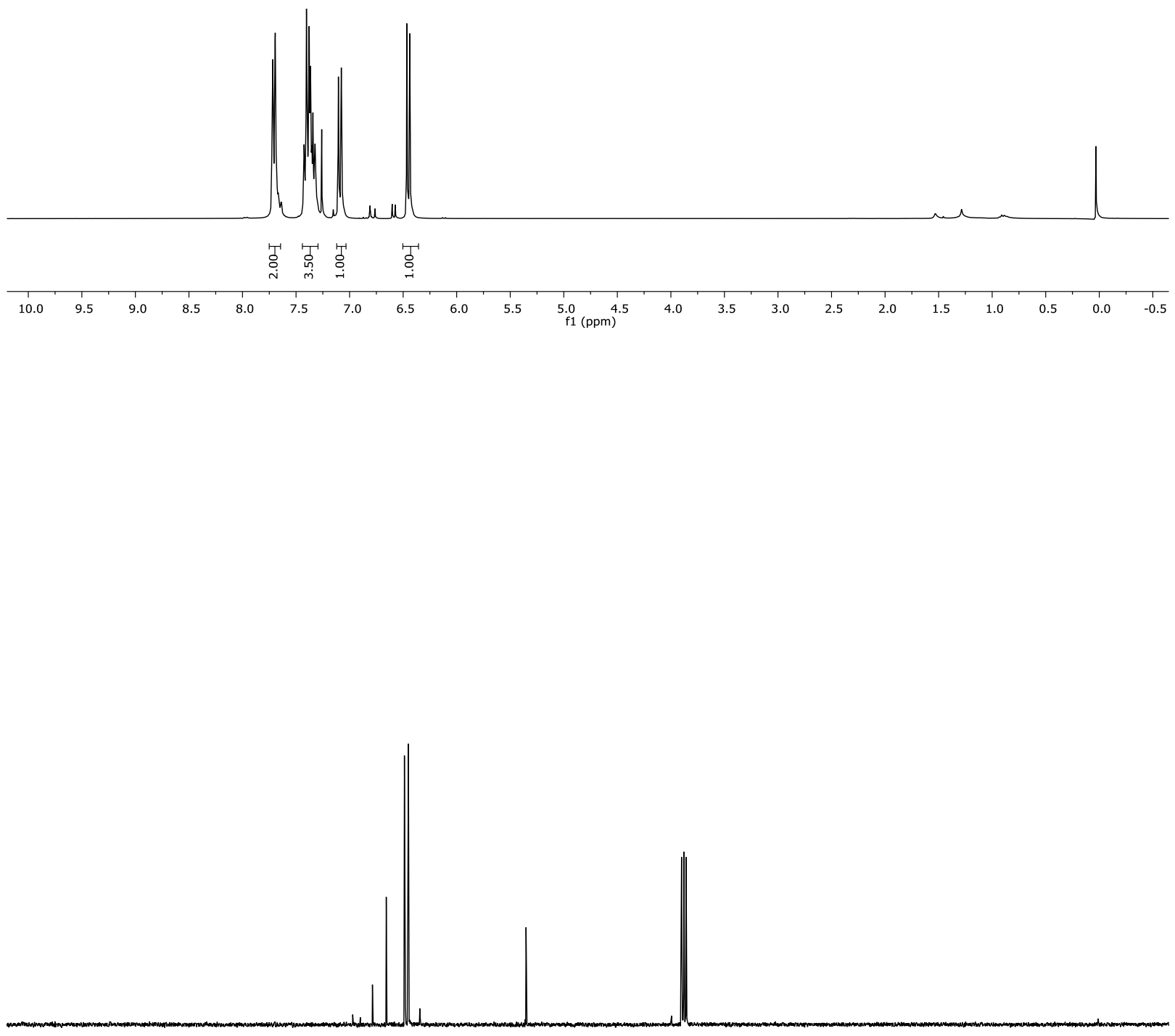

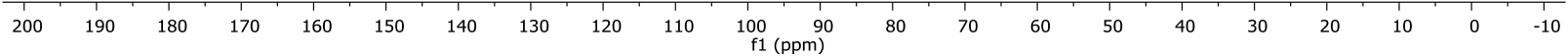




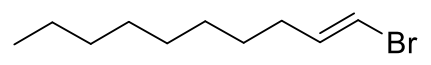

$11 \mathrm{i}$
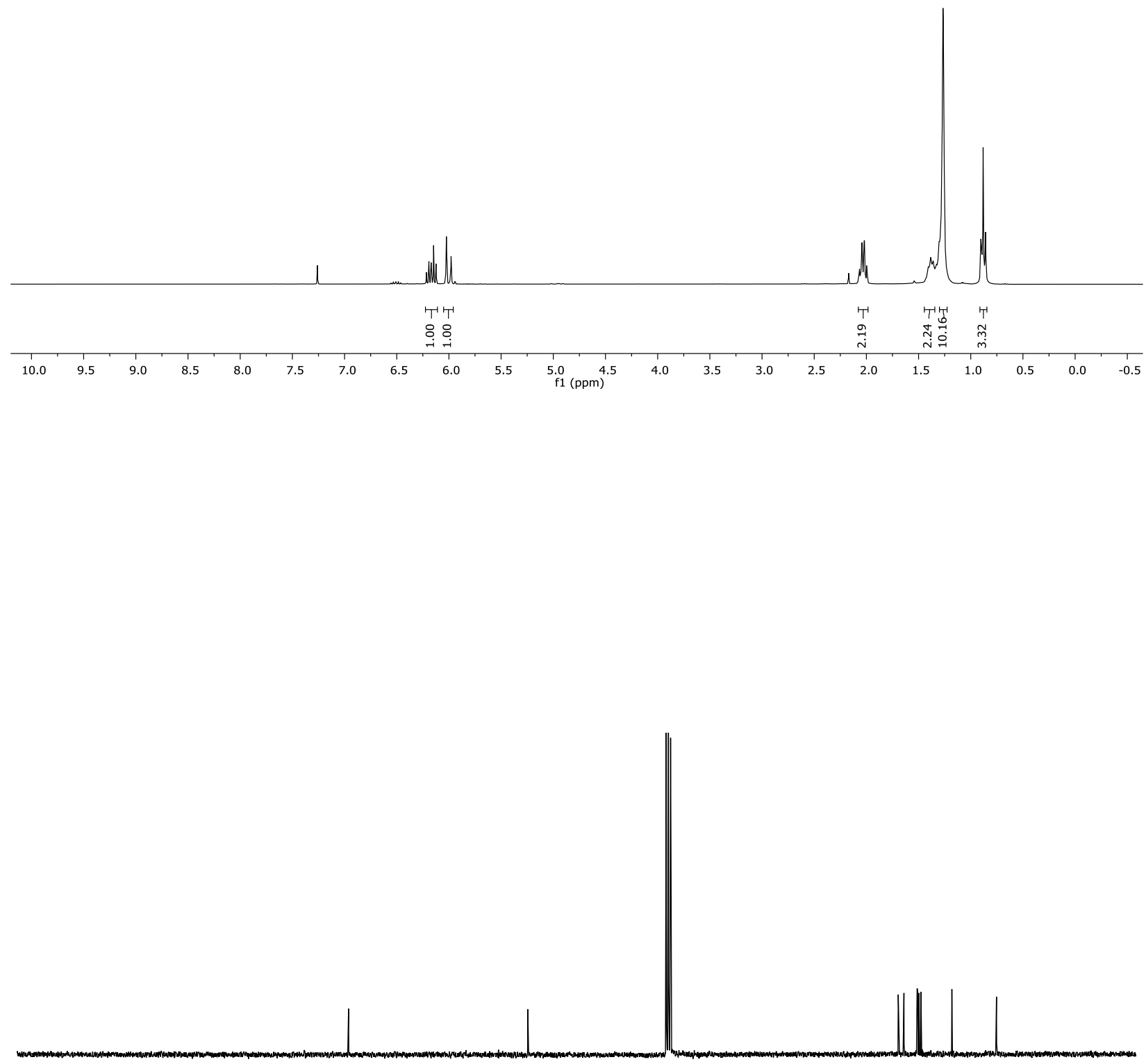

$\begin{array}{llllllllllllllllllllllll}200 & 190 & 180 & 170 & 160 & 150 & 140 & 130 & 120 & 110 & 100 & 90 & 80 & 70 & 60 & 50 & 40 & 30 & 20 & 10 & 0 & -10\end{array}$ 


\section{TBDPSO $ح \mathrm{Br}$}

11j
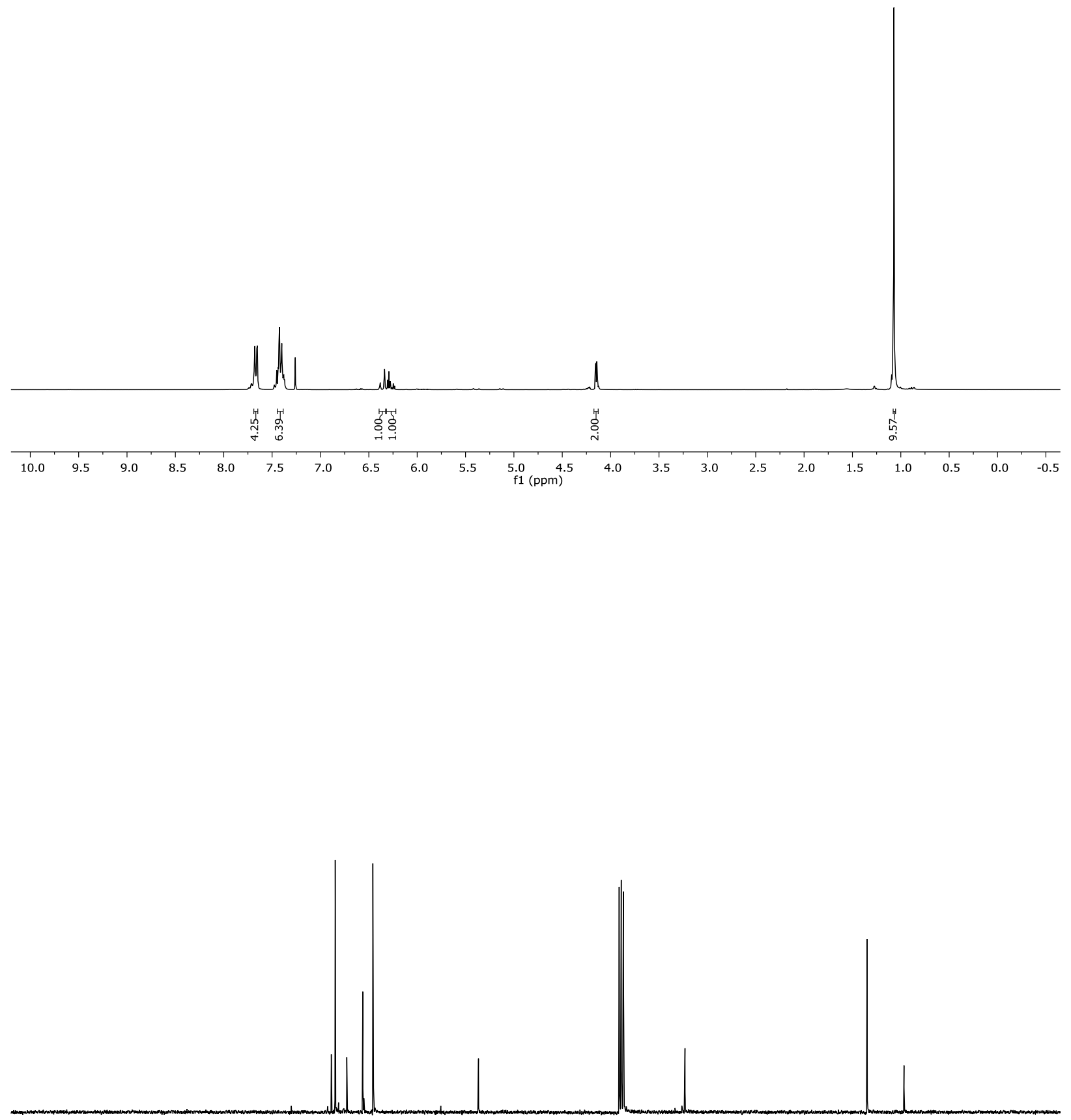

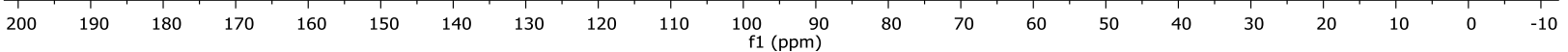




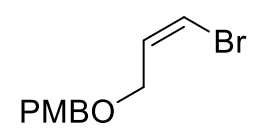

111
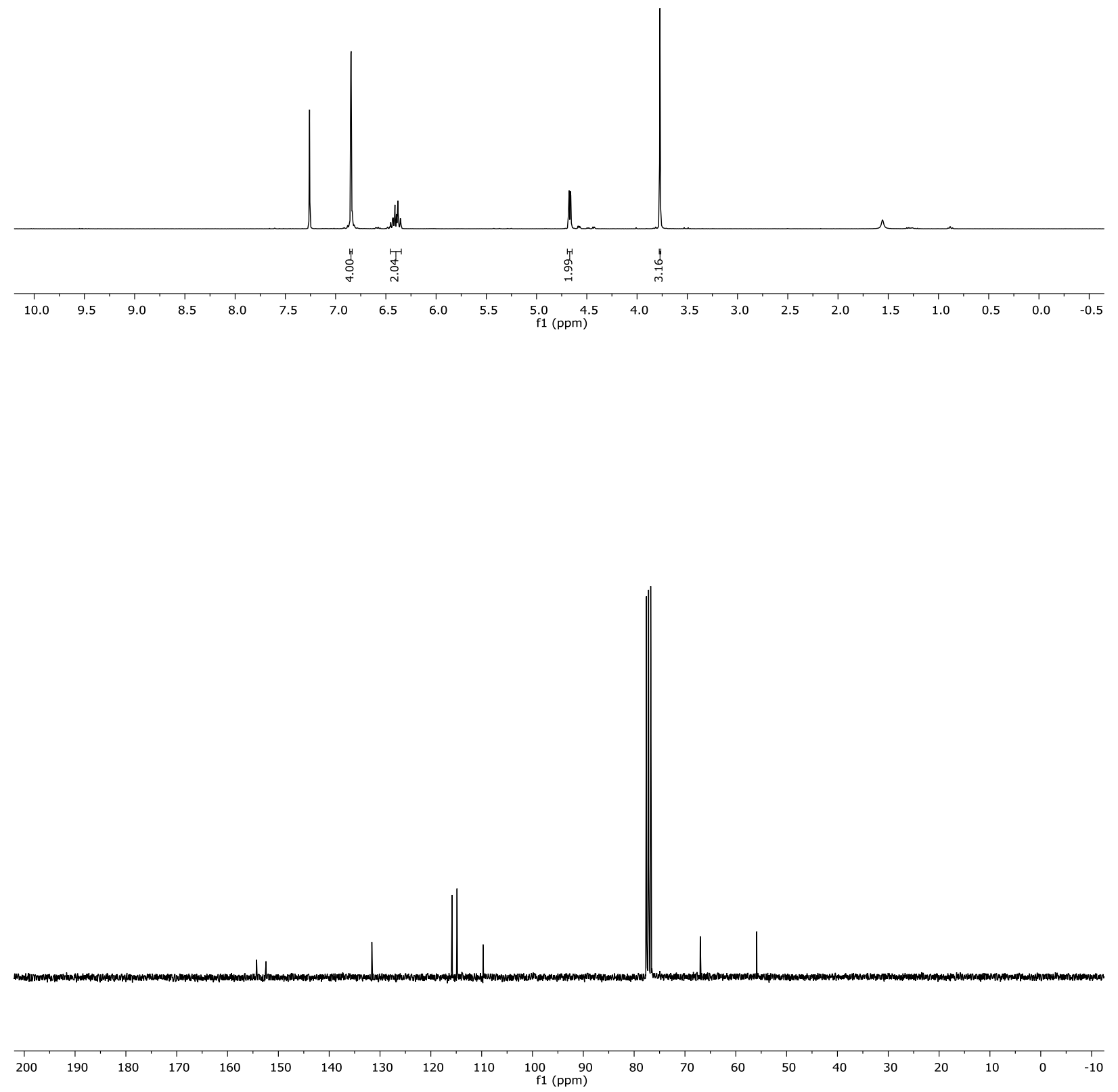


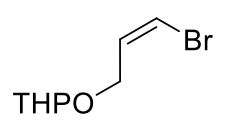

$11 \mathrm{~m}$
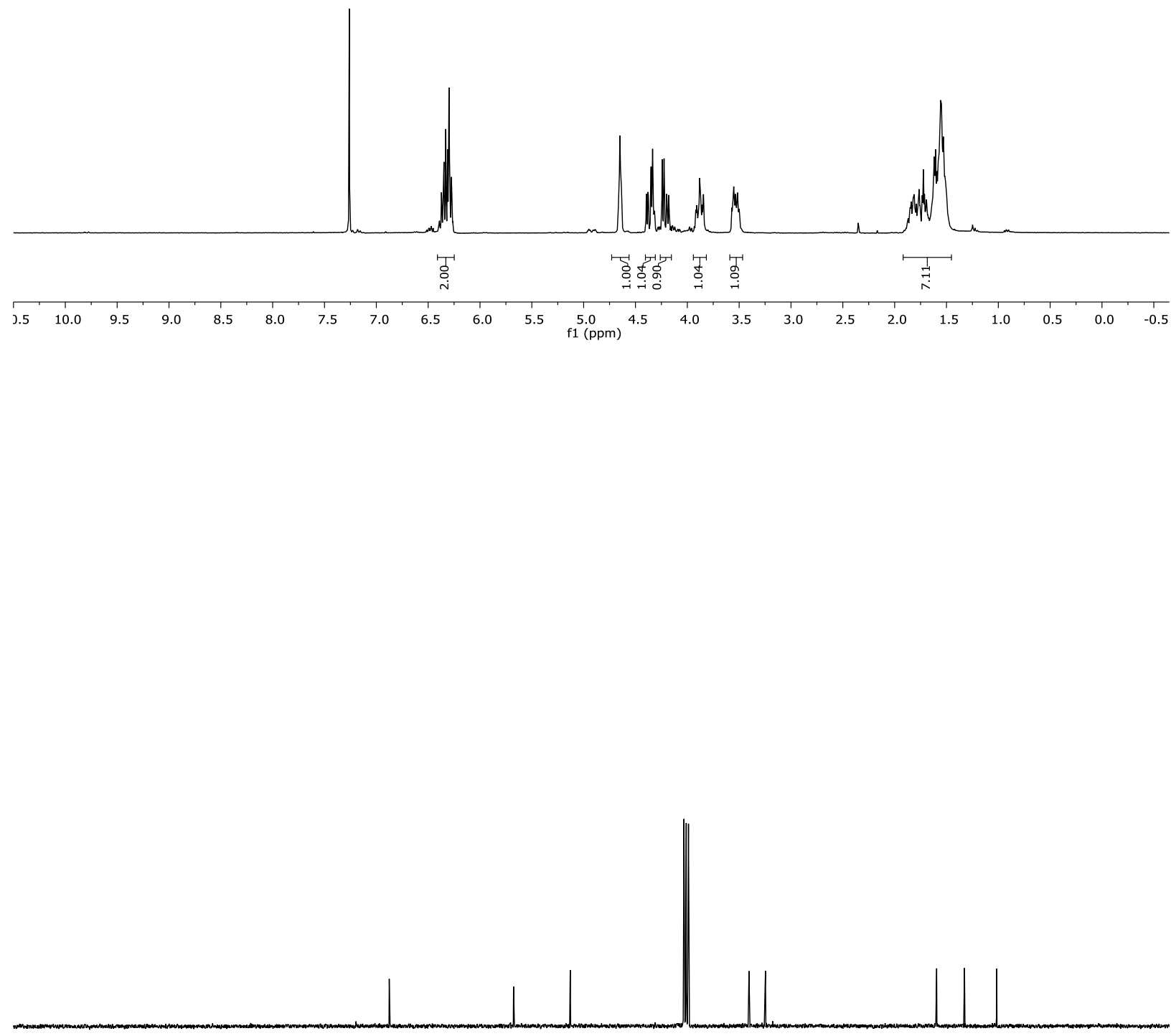

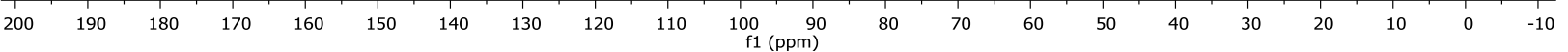




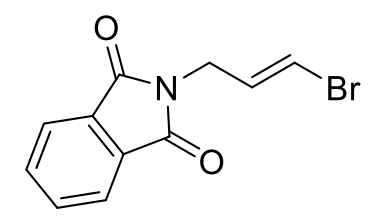

$11 n$
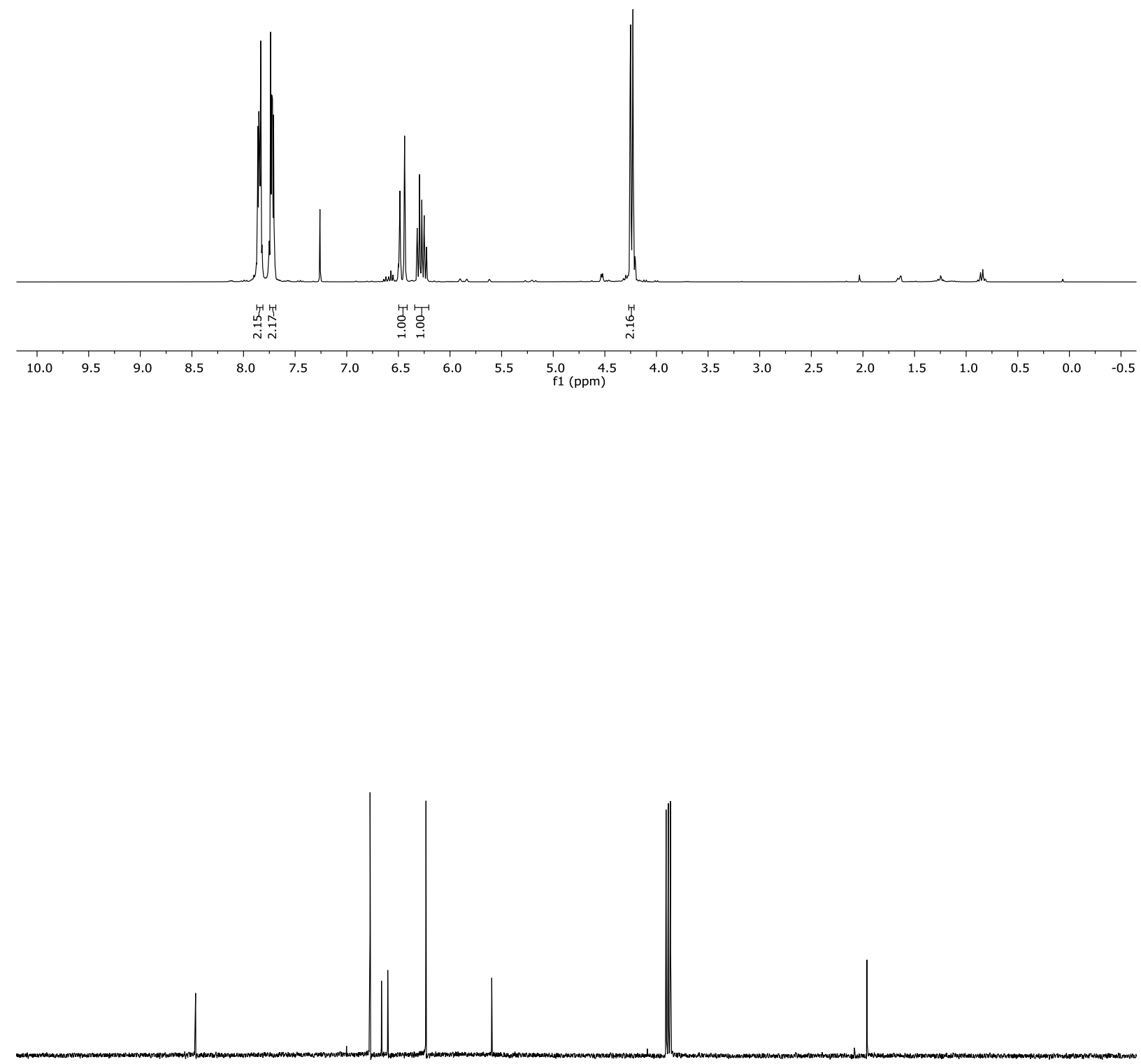

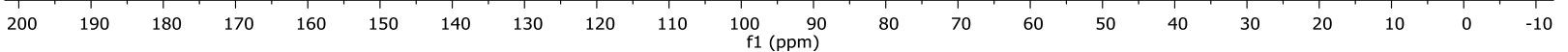




$$
\overbrace{\mathrm{CO}} \mathrm{Et}
$$

110
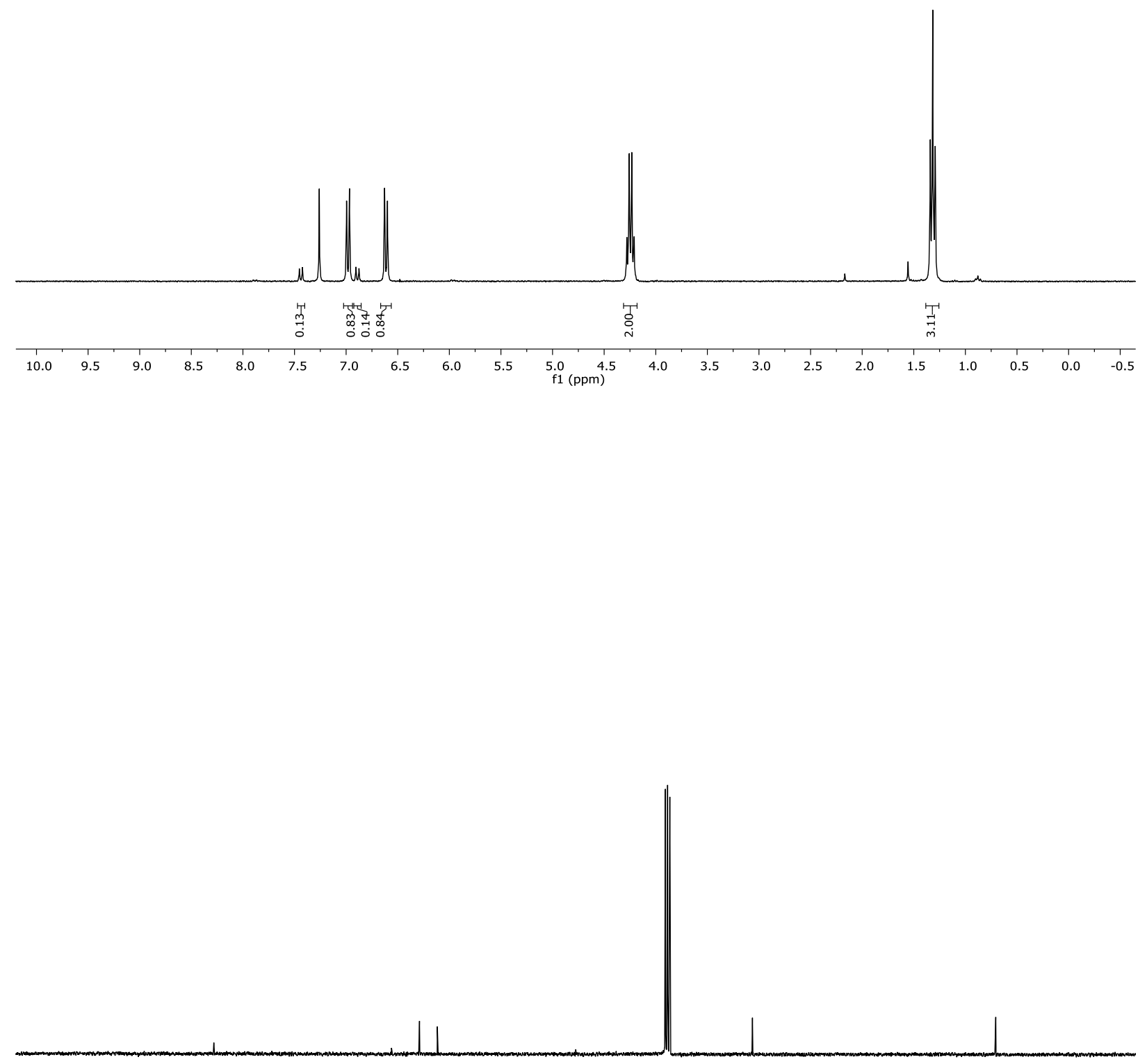

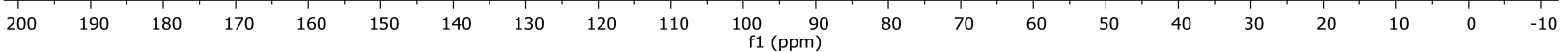




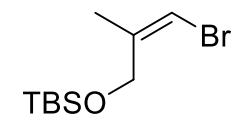

$11 p$
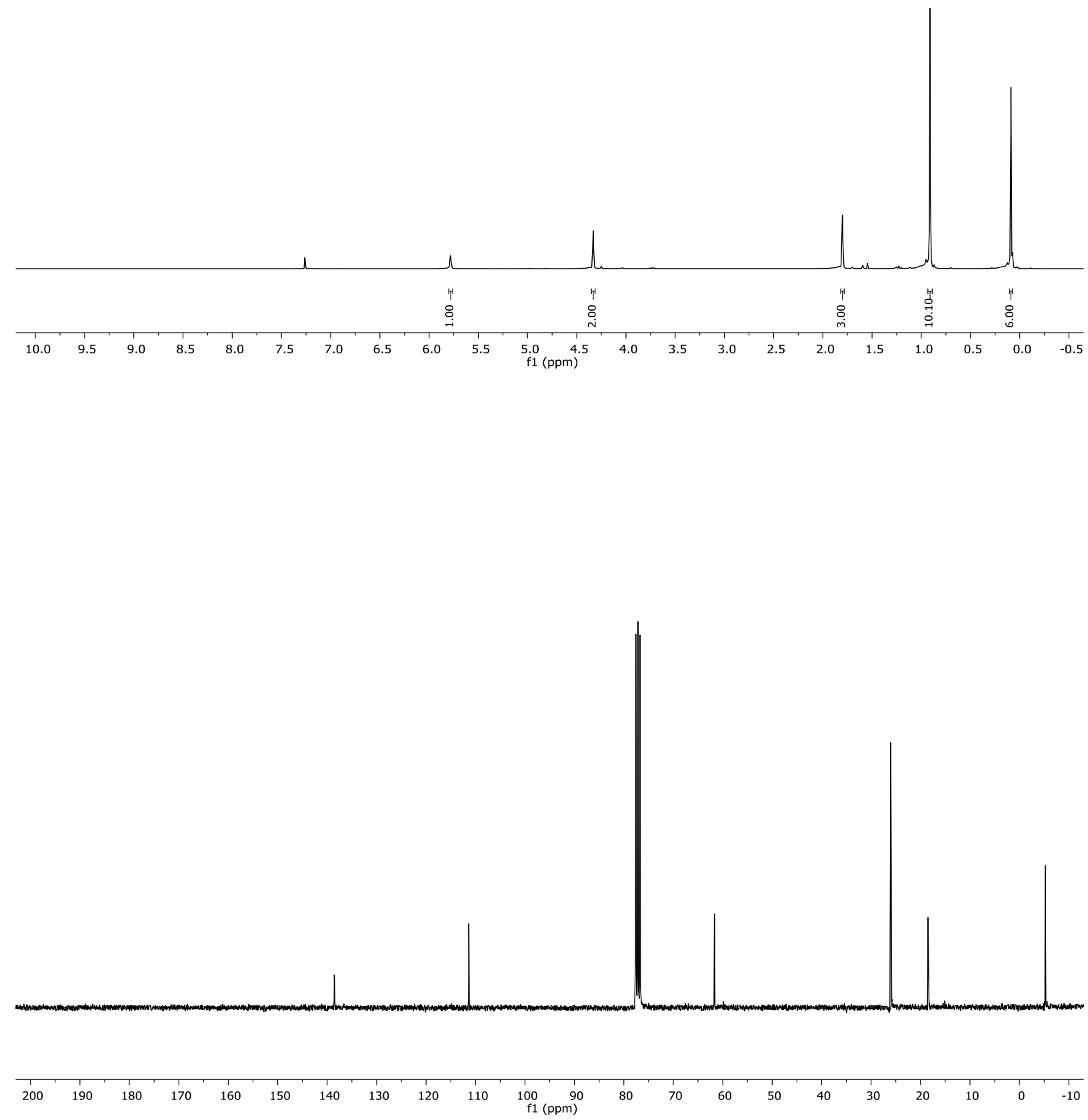


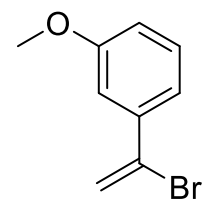

$11 q$
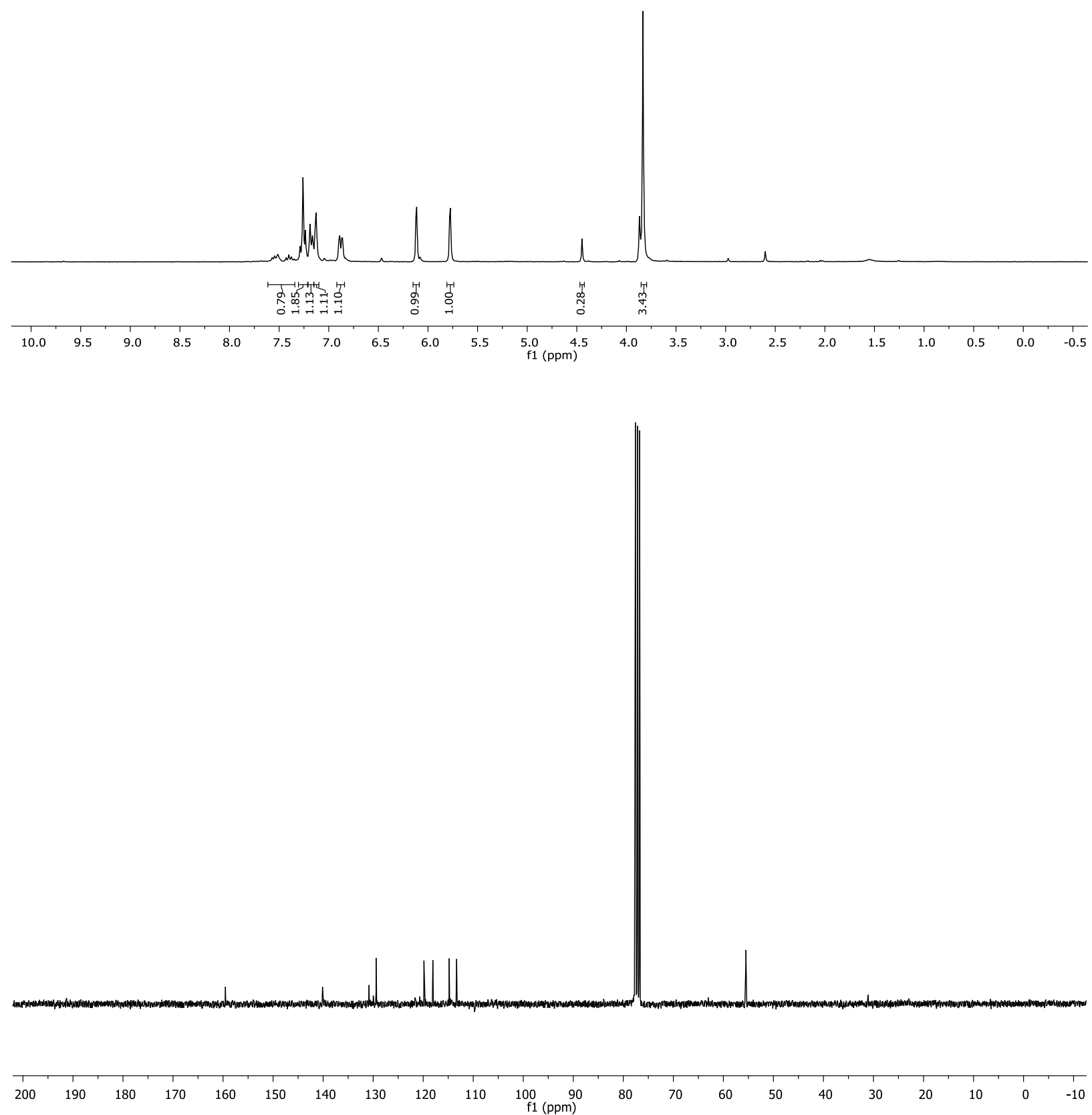
<smiles>C=C(Br)CCCCCCCC</smiles>

$11 r$
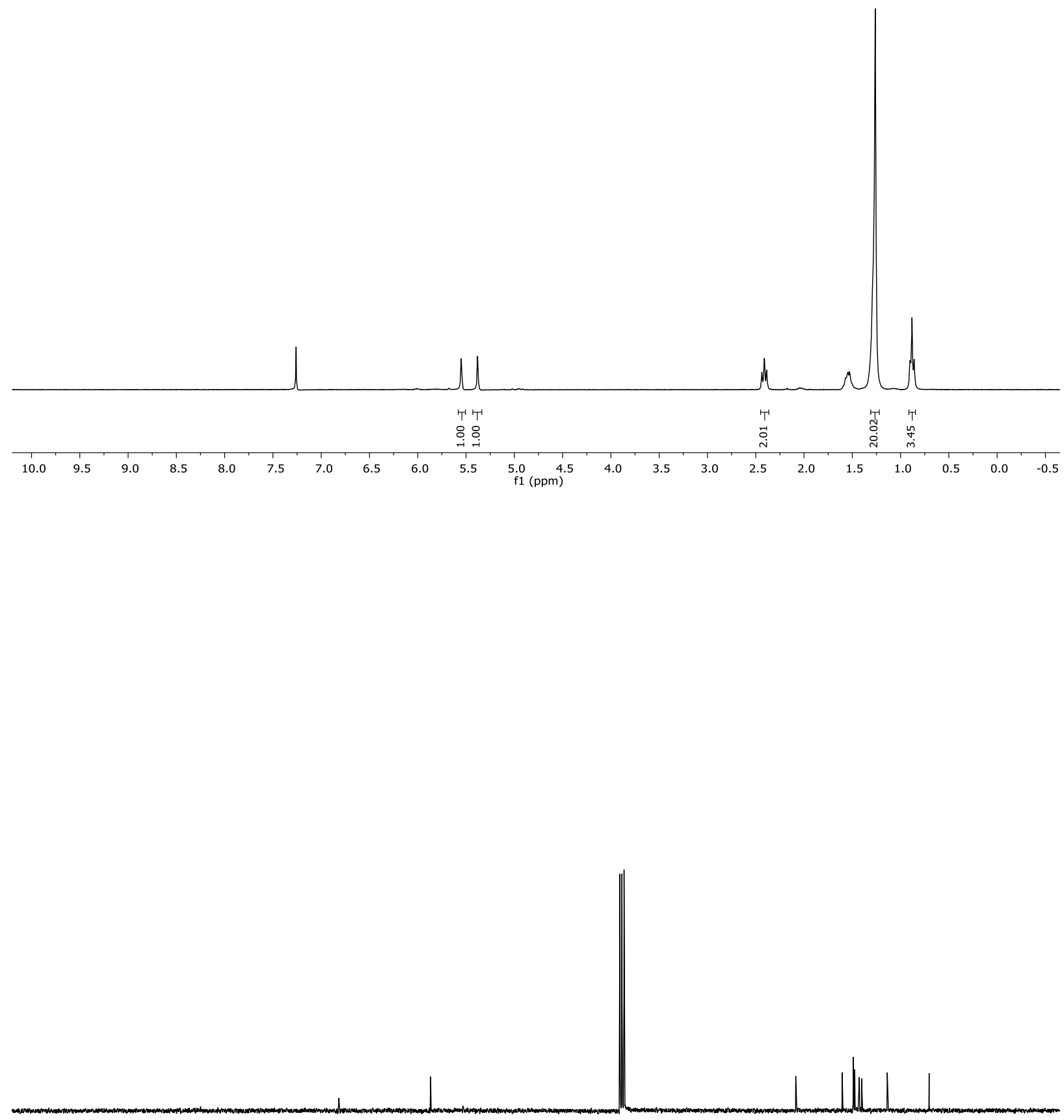

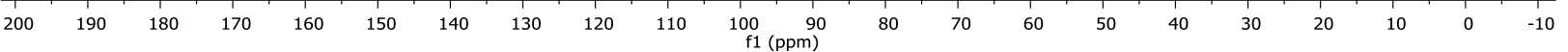




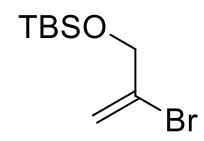

$11 \mathrm{~s}$
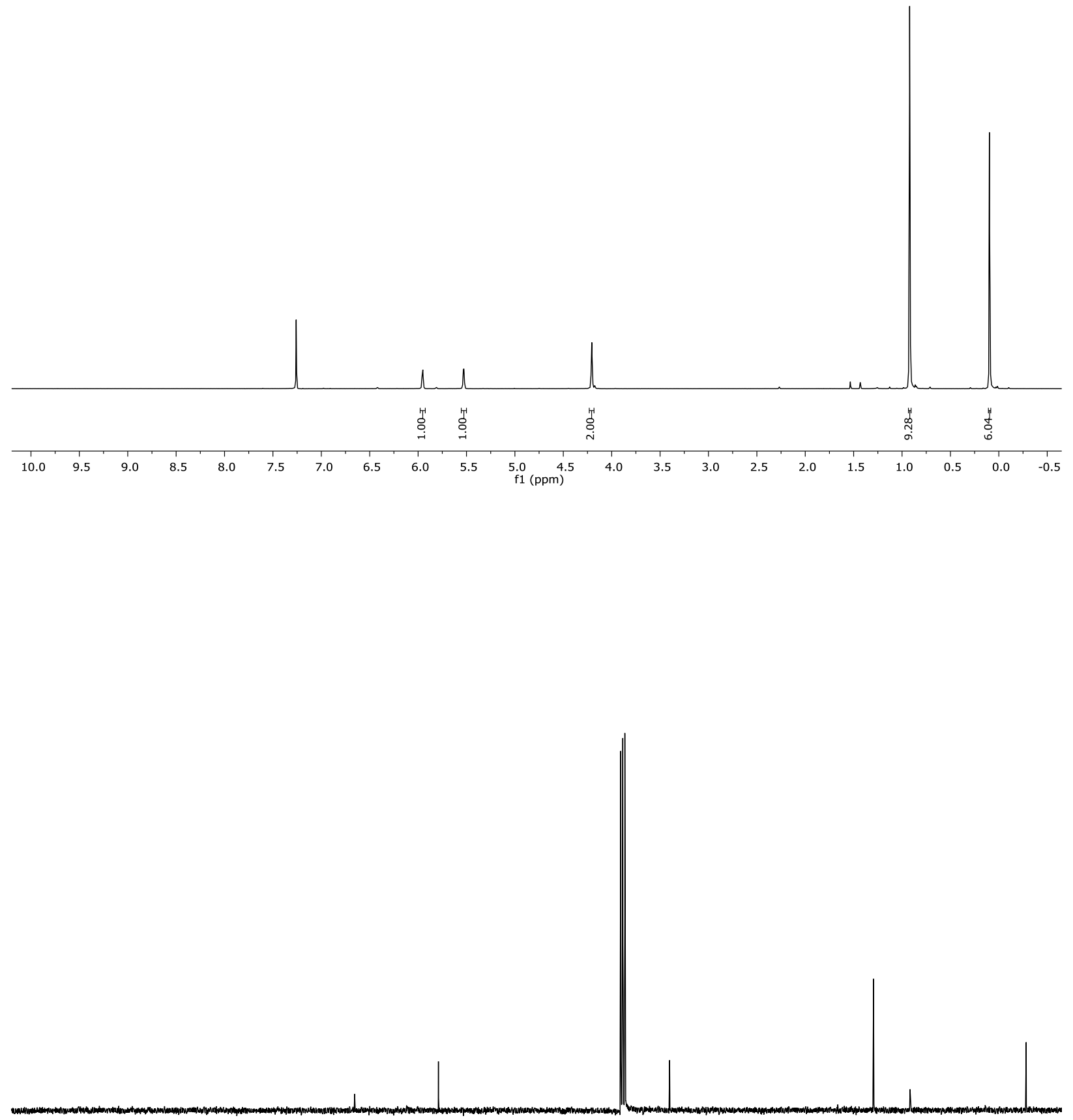

150

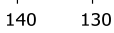

120

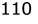

100
$\mathrm{f} 1(\mathrm{ppm})$

80

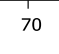

60

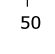

40
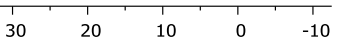


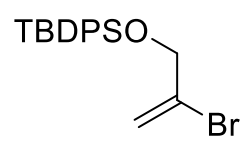

$11 t$
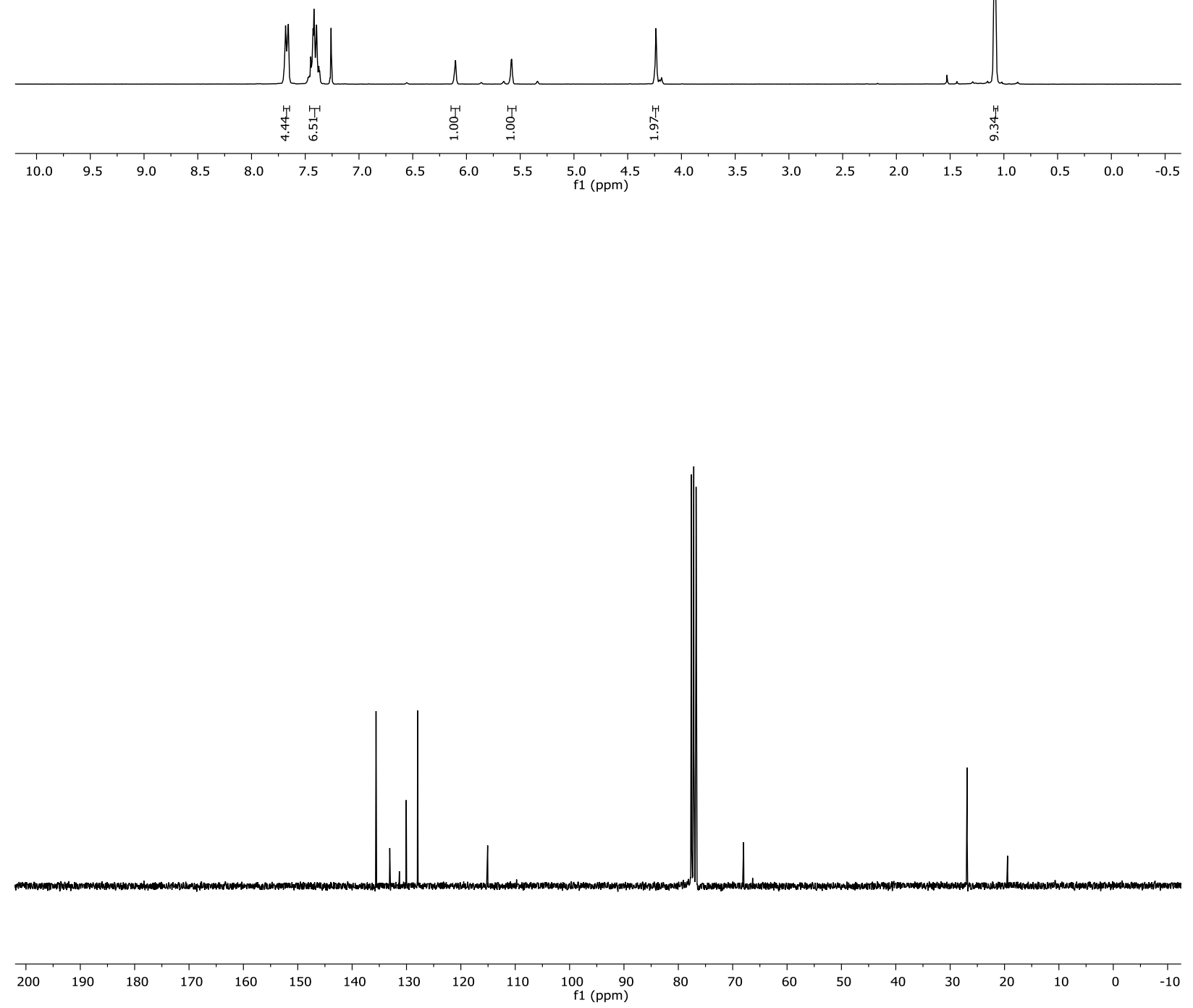


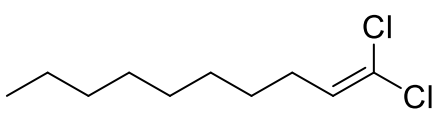

$13 a$
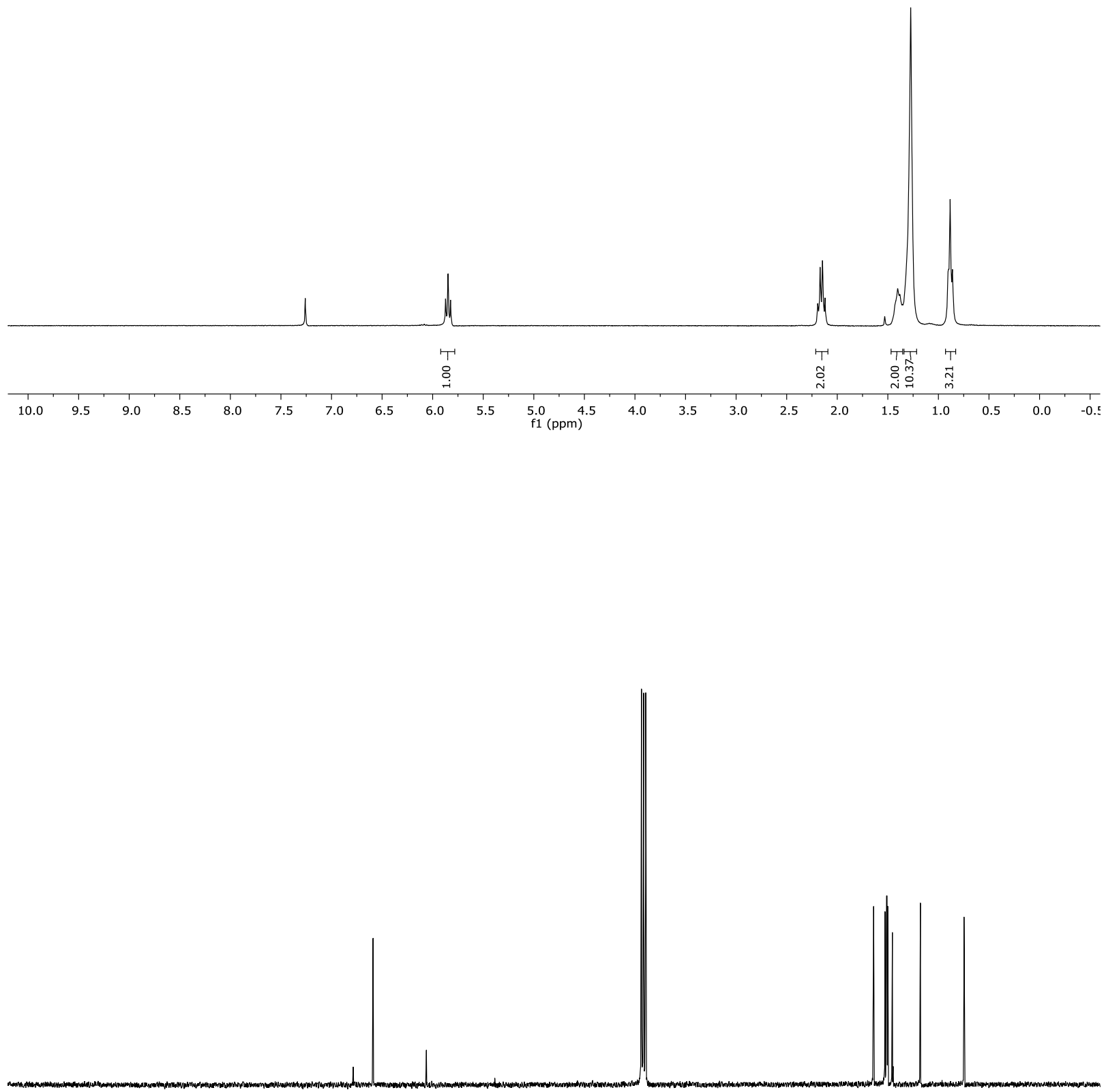

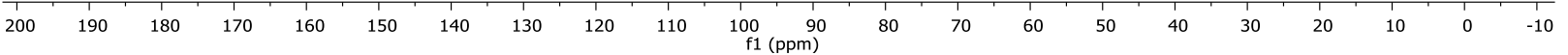




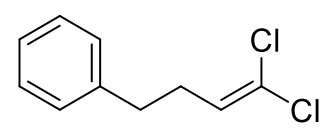

$13 b$
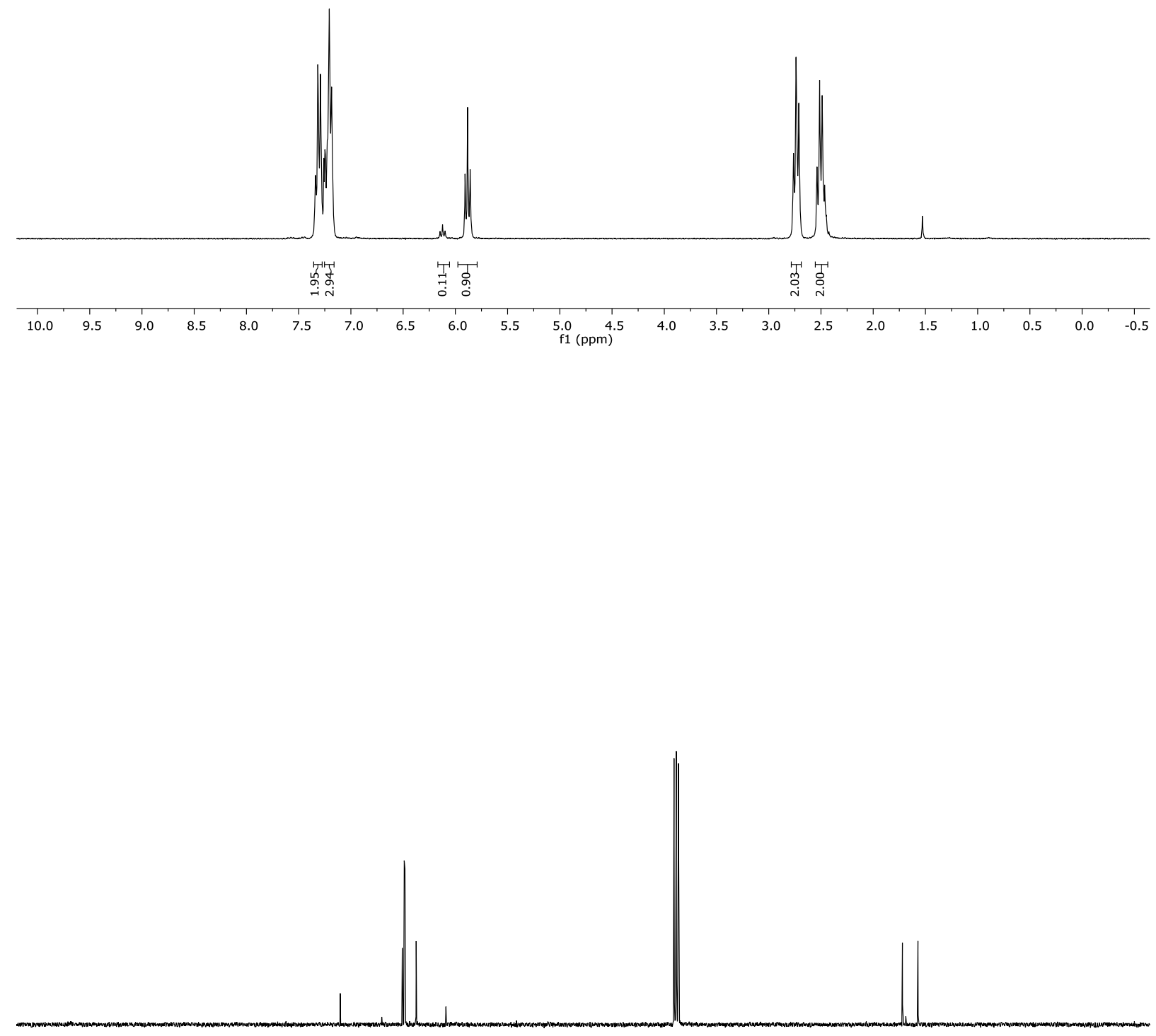

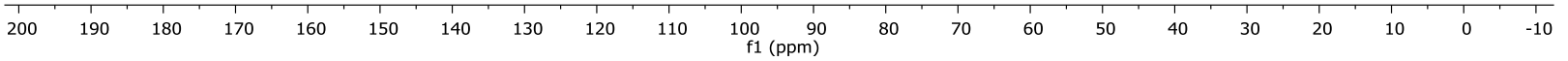




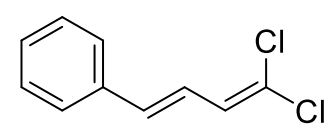

$13 c$
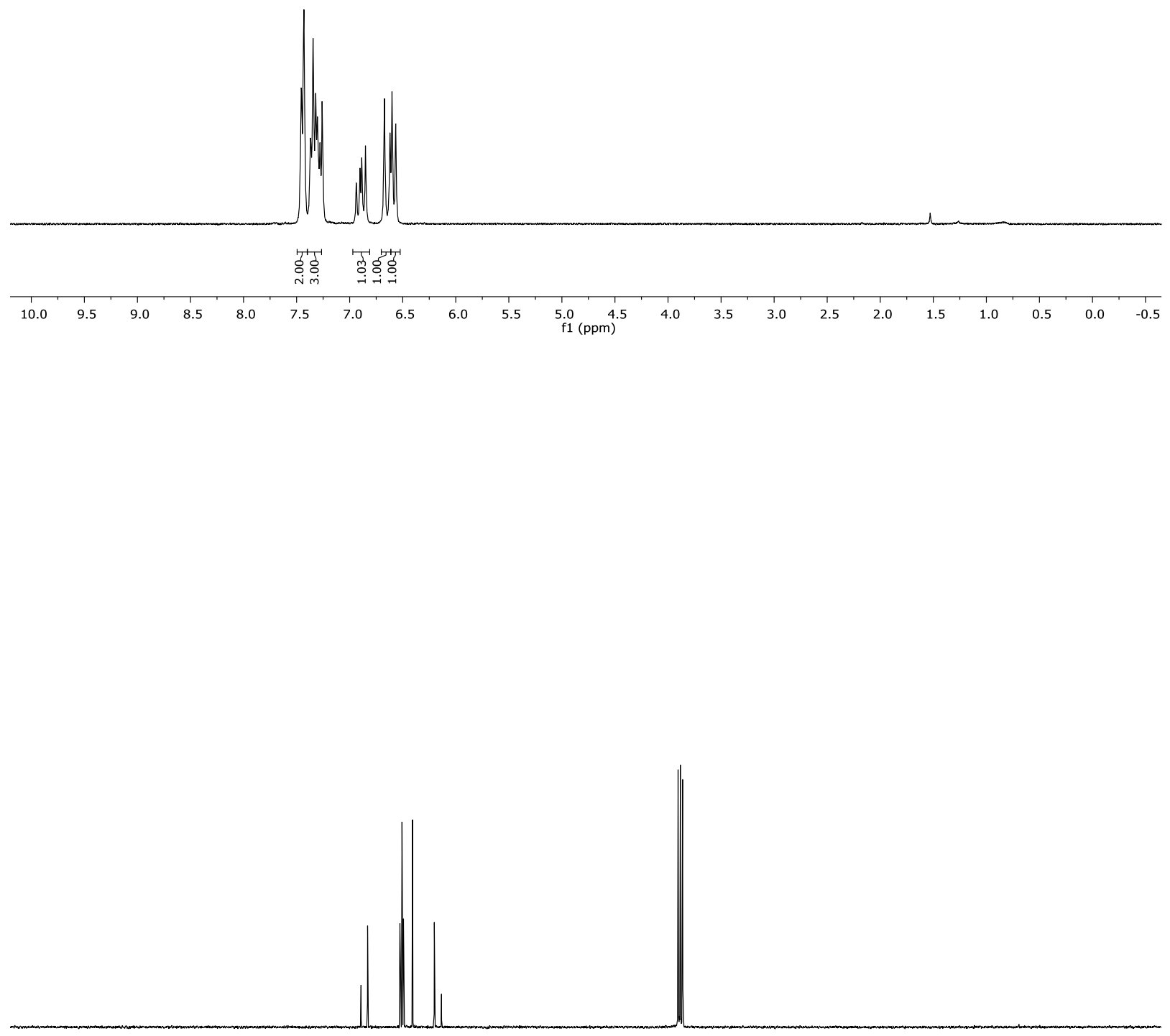

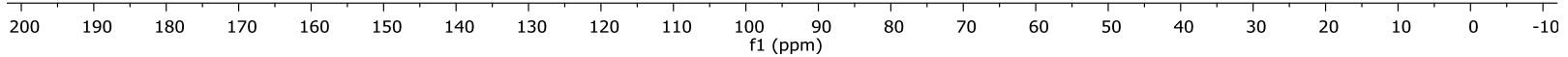




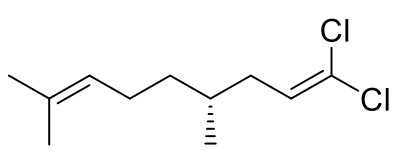

$13 d$
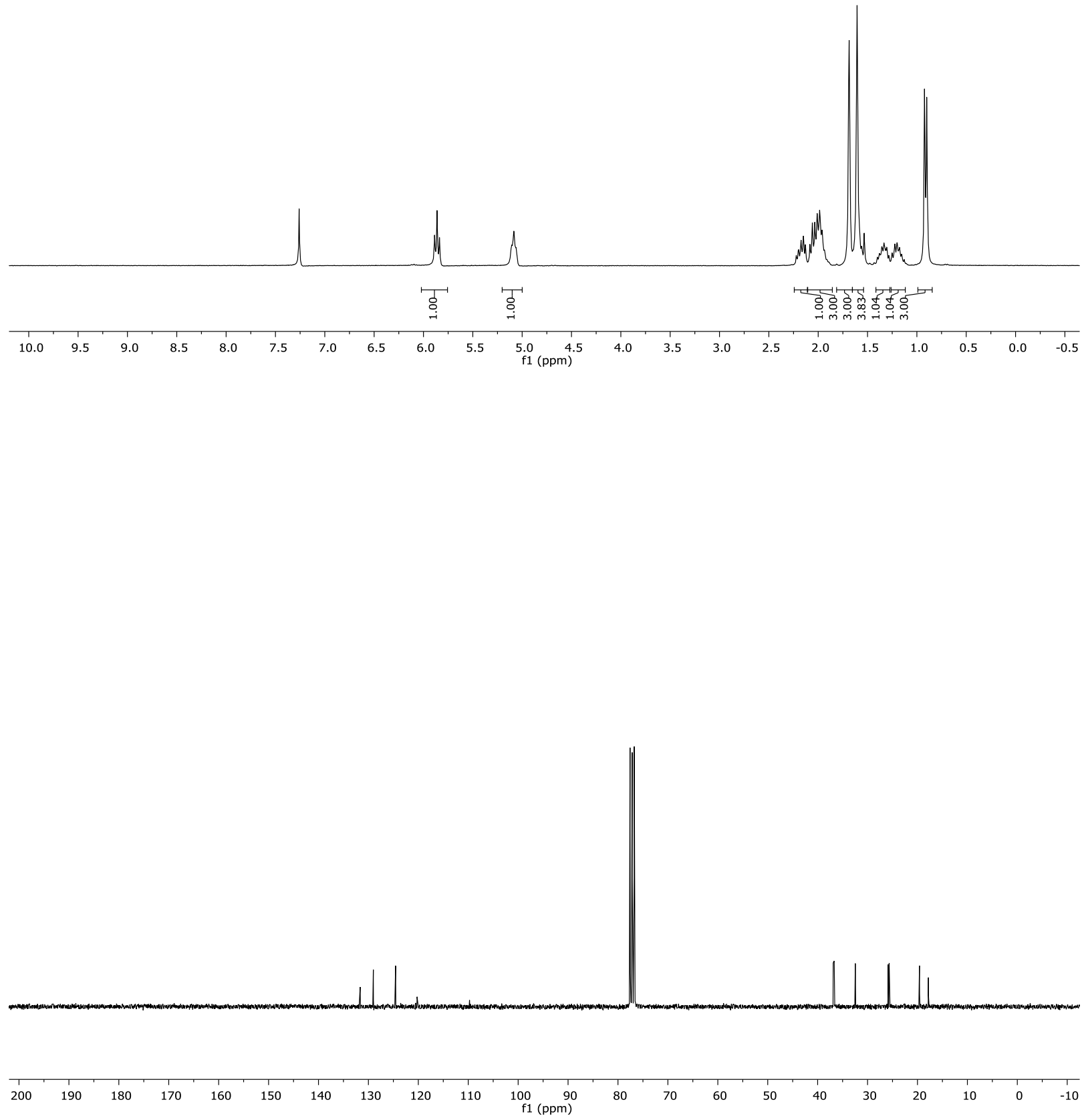